Stochastic Systems

2011, Vol. 1, No. 1, 146-208

DOI: $10.1214 / 10-$ SSY022

\title{
REFLECTING BROWNIAN MOTION IN TWO DIMENSIONS: EXACT ASYMPTOTICS FOR THE STATIONARY DISTRIBUTION
}

\author{
By J. G. Dai and Masakiyo Miyazawa \\ Georgia Institute of Technology and Tokyo University of Science
}

We consider a two-dimensional semimartingale reflecting Brownian motion (SRBM) in the nonnegative quadrant. The data of the SRBM consists of a two-dimensional drift vector, a $2 \times 2$ positive definite covariance matrix, and a $2 \times 2$ reflection matrix. Assuming the SRBM is positive recurrent, we are interested in tail asymptotic of its marginal stationary distribution along each direction in the quadrant. For a given direction, the marginal tail distribution has the exact asymptotic of the form $b x^{\kappa} \exp (-\alpha x)$ as $x$ goes to infinity, where $\alpha$ and $b$ are positive constants and $\kappa$ takes one of the values $-3 / 2,-1 / 2,0$, or 1 ; both the decay rate $\alpha$ and the power $\kappa$ can be computed explicitly from the given direction and the SRBM data.

A key tool in our proof is a relationship governing the moment generating function of the two-dimensional stationary distribution and two moment generating functions of the associated one-dimensional boundary measures. This relationship allows us to characterize the convergence domain of the two-dimensional moment generating function. For a given direction $c$, the line in this direction intersects the boundary of the convergence domain at one point, and that point uniquely determines the decay rate $\alpha$. The one-dimensional moment generating function of the marginal distribution along direction $c$ has a singularity at $\alpha$. Using analytic extension in complex analysis, we characterize the precise nature of the singularity there. Using that characterization and complex inversion techniques, we obtain the exact asymptotic of the marginal tail distribution.

1. Introduction. This paper is concerned with the asymptotic tail behavior of the stationary distributions of two-dimensional semimartingale reflecting Brownian motions (SRBMs). As background for this study, we briefly discuss general multidimensional SRBMs. They are diffusion processes that arise as approximations for queueing networks of various kinds, cf. $[12]$ and $[30,31]$. The state space for a $d$-dimensional SRBM $Z=\{Z(t), t \geq$ $0\}$ is $\mathbb{R}_{+}^{d}$ (the non-negative orthant). The data of the process are a drift vec-

Received November 2010.

AMS 2000 subject classifications: Primary 60J60, 60K65; secondary 60K25, 60G42, 90C33.

Keywords and phrases: Diffusion process, heavy traffic, queueing network, tail behavior, large deviations. 
tor $\mu$, a non-singular covariance matrix $\Sigma$, and a $d \times d$ "reflection matrix" $R$ that specifies boundary behavior. In the interior of the orthant, $Z$ behaves as an ordinary Brownian motion with parameters $\mu$ and $\Sigma$, and $Z$ is pushed in direction $R^{j}$ whenever the boundary surface $\left\{z \in \mathbb{R}_{+}^{d}: z_{j}=0\right\}$ is hit, where $R^{j}$ is the $j$ th column of $R$, for $j=1, \ldots, d$. To make this description more precise, one represents $Z$ in the form

$$
Z(t)=X(t)+R Y(t), \quad t \geq 0,
$$

where $X$ is an unconstrained Brownian motion with drift vector $\mu$, covariance matrix $\Sigma$, and $Z(0)=X(0) \in \mathbb{R}_{+}^{d}$, and $Y$ is a $d$-dimensional process with components $Y_{1}, \ldots, Y_{d}$ such that

(1.2) $Y$ is continuous and non-decreasing with $Y(0)=0$,

(1.3) $Y_{j}$ only increases at times $t$ for which $Z_{j}(t)=0, \quad j=1, \ldots, d, \quad$ and (1.4) $Z(t) \in \mathbb{R}_{+}^{d}, \quad t \geq 0$.

The complete definition of the diffusion process $Z$ will be given in Section A.1.

A $d \times d$ matrix $R$ is said to be an $\mathcal{S}$-matrix if there exists a $d$-vector $w \geq 0$ such that $R w>0$ (or equivalently, if there exists $w>0$ such that $R w>0$ ), and $R$ is said to be completely-S $\mathcal{S}$ if each of its principal sub-matrices is an $\mathcal{S}$-matrix. (For a vector $v$, we write $v>0$ to mean that each component of $v$ is positive, and we write $v \geq 0$ to mean that each component of $v$ is nonnegative.) Taylor and Williams [29] and Reiman and Williams [27] show that for a given data set $(\Sigma, \mu, R)$ with $\Sigma$ being positive definite, there exists an SRBM for each initial distribution of $Z(0)$ if and only if $R$ is completely- $\mathcal{S}$. Furthermore, when $R$ is completely- $\mathcal{S}$, the SRBM $Z$ is unique in distribution for each given initial distribution. A necessary condition of the existence of the stationary distribution for $Z$ is

$$
R \text { is non-singular and } R^{-1} \mu<0 .
$$

If $R$ is an $\mathcal{M}$-matrix as defined in Chapter 6 of [2], then (1.5) is known to be necessary and sufficient for the existence and uniqueness of a stationary distribution of $Z$; Harrison and Williams [13] prove that result and explain how the $\mathcal{M}$-matrix structure arises naturally in queueing network applications. A square matrix is said to be a $\mathcal{P}$-matrix if all of its principal minors are positive (that is, each principal submatrix of $R$ has a positive determinant). Obviously, $\mathcal{M}$-matrix is a special case of $\mathcal{P}$-matrix. For a two-dimensional SRBM, Harrison and Hasenbein [11] show that condition (1.5) and $R$ being a $\mathcal{P}$ matrix are necessary and sufficient for the existence of a stationary distribution. 
In this paper we are concerned with two-dimensional SRBMs. Throughput this paper, we assume $R$ is a $\mathcal{P}$ matrix and $(R, \mu)$ satisfy (1.5). Therefore, such an SRBM has a unique stationary distribution. We are interested in the asymptotic tail behavior of the stationary distribution. Let $Z(\infty) \equiv$ $\left(Z_{1}(\infty), Z_{2}(\infty)\right)$ be a random vector that has the stationary distribution of the SRBM. Let $c \in \mathbb{R}_{+}^{2}$ be a directional vector, i.e., a nonnegative vector in $\mathbb{R}^{2}$ such that $\|c\| \equiv \sqrt{\langle c, c\rangle}=1$, where $\langle x, y\rangle$ is the inner product of vectors $x$ and $y$. We are interested in the asymptotic tail behavior of $\mathbb{P}\{\langle c, Z(\infty)\rangle \geq x\}$ as $x \rightarrow \infty$. For a given direction $c$, if there exists a function $f_{c}(x)$ such that

$$
\lim _{x \rightarrow \infty} \frac{\mathbb{P}(\langle c, Z(\infty)\rangle \geq x)}{f_{c}(x)}=1,
$$

then the function $\mathbb{P}(\langle c, Z(\infty)\rangle \geq x)$ is said to have exact asymptotic $f_{c}(x)$. Our major interest is to compute exact asymptotics in any given direction $c$ from the primitive data $(\Sigma, \mu, R)$. In this paper we will prove that, for each $c \in \mathbb{R}_{+}^{2}, f_{c}(x)$ can be taken to be

$$
f_{c}(x)=b x^{\kappa_{c}} e^{-\alpha_{c} x},
$$

for some constant $b>0$. That is,

$$
\mathbb{P}\{\langle c, Z(\infty)\rangle \geq x\}=b x^{\kappa_{c}} e^{-\alpha_{c} x}+o\left(x^{\kappa_{c}} e^{-\alpha_{c} x}\right) \quad \text { as } x \rightarrow \infty .
$$

The exponent $\alpha_{c}>0$ is known as the decay rate. The decay rate $\alpha_{c}$ and the constant $\kappa_{c}$ can be computed explicitly from the primitive data, and $\kappa_{c}$ must take one of the values $-3 / 2,-1 / 2,0$, or 1 . The complete results are stated in Section 2.

Although our major interest is in the exact asymptotics of the tail probability, for many cases we have actually obtained the exact asymptotic for the density of the random variable $\langle c, Z(\infty)\rangle$. In these cases, it will be proven that for each direction $c$, the density $p_{c}(x)$ exists, $p_{c}(x)$ is continuous in $x$ on $[0, \infty)$, and

$$
p_{c}(x)=\alpha_{c} b x^{\kappa_{c}} e^{-\alpha_{c} x}+o\left(x^{\kappa_{c}} e^{-\alpha_{c} x}\right) \quad \text { as } x \rightarrow \infty,
$$

with the same $b, \kappa_{c}$ and $\alpha_{c}$ as in (1.7). In these cases, we first establish (1.8) and then obtain (1.7) from (1.8) as shown in Lemma D.5. In other cases, we are not able to establish (1.8) and will work with the tail probabilities directly.

To get the exact asymptotics, we use the moment generating function of the random variable $\langle c, Z(\infty)\rangle$. Let

$$
\psi_{c}(\lambda)=\mathbb{E}\left(e^{\lambda\langle c, Z(\infty)\rangle}\right) .
$$


Intuitively, the decay rate in (1.6) should be

$$
\sup \left\{\lambda \geq 0: \psi_{c}(\lambda)<\infty\right\}
$$

(This will be proved as a consequence of our Theorem 2.1.) Equivalently, the decay rate is the first singular point $\psi_{c}(z)$ on the real axis when $\psi_{c}(z)$ is viewed as a complex function of $z$. Let

$$
\varphi\left(\theta_{1}, \theta_{2}\right)=\mathbb{E}\left(e^{\langle\theta, Z(\infty)\rangle}\right)
$$

be the two-dimensional moment generating function of

$$
Z(\infty)=\left(Z_{1}(\infty), Z_{2}(\infty)\right)
$$

Since $\psi_{c}(\lambda)=\varphi(\lambda c)$ for $\lambda \in \mathbb{R}$, the singularity of $\varphi(z c)$ is used to determine the decay rate for each direction $c$. It turns out the singularity of $\varphi(z c)$ allows us to apply a complex inversion technique to get the exact asymptotics.

To find the singularities of moment generating functions, one tries to derive closed form expressions of these functions. This is the approach used in [20]. That paper studied a tandem queue whose input is driven by a Lévy process that does not have negative jumps; this input process includes Brownian motion as a special case. However, exact asymptotic results there have not been fully proved yet (see Section 1 of [26] for some more discussions). For a two-dimensional reflecting random walk on the lattice with skip-free transitions, the book [8] (see also [16]) derived certain expressions for the generating function of the stationary distribution from a certain stationary equation that is analogous to our (2.3). Their techniques either use analytic extensions on Riemann surfaces or reduce the problem to the Riemann boundary value problem. These techniques may be useful to our problem in this paper, but we have not explored them here.

A recent paper [26] pioneered another analytic approach for a special case of SRBM. That SRBM arises from a similar tandem queue as in $[10,19]$ but with an intermediate input, for which an explicit form is only partially available for the moment generating function of the stationary distribution. The authors first find the convergence domain of the moment generating function, namely,

$$
\mathcal{D}=\text { interior of }\left\{\theta \in \mathbb{R}^{2}: \varphi(\theta)<\infty\right\} .
$$

From the convergence domain, it is relatively easy to find the singularities of $\varphi(z c)$ for each direction $c$. In this paper we take this analytic approach and show its full potential. As in [26], we consider some boundary moment 
generating functions that capture the reflections on the boundary faces. Unlike [26], we need to carefully study a relationship governing these moment generating functions. In this paper we do not assume any a priori information on the stationary distribution, whereas in [26] the marginal stationary distribution corresponding to the first node of the tandem queue is known. This forces us to seek a precise relationship among these moment generating functions (Lemma 4.1). This relationship is critical for us to characterize the convergence domain in Theorem 2.1.

Once the convergence domain is obtained, we employ analytic function theory to arrive at our main results, Theorems 2.2 and 2.3. Interestingly, it turns out that we can go beyond these results for some cases, obtaining a refinement of the exact asymptotics in (1.7). For example, the refinement can take the form

$$
\begin{aligned}
\mathbb{P}\{\langle c, Z(\infty)\rangle \geq & x\}=b_{c} x^{\kappa_{c}} e^{-\alpha_{c} x}+b_{d} x^{\kappa_{d}} e^{-\alpha_{d} x} \\
& +o\left(\min \left(x^{\kappa_{c}} e^{-\alpha_{c} x}, x^{\kappa_{d}} e^{-\alpha_{d} x}\right)\right) \quad \text { as } x \rightarrow \infty,
\end{aligned}
$$

where $0<\alpha_{c} \leq \alpha_{d}$ and $b_{c}, b_{d}, \kappa_{c}$ and $\kappa_{d}$ are some constants. We will briefly discuss this type of refinement in Section 8.

Determining exact asymptotics for two-dimensional SRBMs has been a difficult problem. Harrison and Williams [14] proved that when $\Sigma$ and $R$ satisfy the so-called skew symmetry condition, the stationary distribution of a $d$-dimensional SRBM has a product-form, each marginal being exponential. As a consequence, when the skew symmetry condition is satisfied, the tail asymptotic function $f_{c}(x)$ has the form $x^{\kappa} e^{-\alpha_{c} x}$, where $\kappa$ takes one of the integers in $0,1, \ldots, d-1$. Dieker and Moriarty [4] proved that when $d=2$ and a certain condition on $\Sigma$ and $R$ is satisfied, the two-dimensional stationary density is a finite sum of exponentials. Thus, the exact asymptotic in any direction $c$ is known. For an SRBM arising from a tandem queue, Harrison [10] derives an explicit form for the two-dimensional stationary density. In this case, the exact asymptotic can also be computed; this is carried out in [19]. Except for these special cases and the one studied in [26], the exact asymptotics for two-dimensional SRBMs are not known. A part of the present results have recently been conjectured by Miyazawa and Kobayashi [25], which also includes conjectures for SRBMs in $d \geq 3$ dimensions.

The analytic approach that is fully explored in this paper is general and should be applicable to discrete time reflecting random walks as well, as long as they are "skip free". When a random walk is not skip free, additional difficulties will show up. In such a case, the Markov additive approach (see, for example, [9]) will likely play a major role, although our analytic approach is still relevant; see $[17,18]$. 
Our results are closely related to the large deviations rate function $I(v)$ for $v \in \mathbb{R}_{+}^{2}$. The rate function $I(v)$ is defined as a lower semi-continuous function that satisfies

$$
\begin{aligned}
& \limsup _{u \rightarrow \infty} \frac{1}{u} \log \mathbb{P}(Z(\infty) \in u B) \leq-\inf _{v \in \bar{B}} I(v), \\
& \liminf _{u \rightarrow \infty} \frac{1}{u} \log \mathbb{P}(Z(\infty) \in u B) \geq-\inf _{v \in B^{\circ}} I(v)
\end{aligned}
$$

for any measurable $B \subset \mathbb{R}_{+}^{2}$, where $\bar{B}$ and $B^{\circ}$ are closure and interior of $B$. When (1.11) and (1.12) are satisfied for a positive recurrent SRBM, it is said that the large deviations principle (LDP) holds with rate function $I(v)$. The LDP is verified by Majewski $[21,22]$ for an SRBM when $R$ is an $\mathcal{M}$ matrix and $R^{-1} \mu<0$. When the latter two conditions are satisfied, the rate function $I(v)$ is characterized as a solution to a variational problem. This $\mathcal{M}$-matrix condition can be relaxed (see, e.g., [6]), but there is no LDP established in the literature when $R$ is a completely- $\mathcal{S}$ matrix. Despite the lack of such an LDP, $[1,11]$ studied the corresponding variational problem and derived an implicit characterization of its solution. Denoting

$$
B_{c}=\left\{x \in \mathbb{R}_{+}^{2} ;\langle c, x\rangle \geq 1\right\},
$$

as a consequence of our results, we have that the limit

$$
-\lim _{u \rightarrow \infty} \frac{1}{u} \log \mathbb{P}\left(Z(\infty) \in u B_{c}\right)
$$

exists and equals to the constant $\alpha_{c}$. Thus, we have verified the large deviations limit for $B_{c}$, and the decay rate $\alpha_{c}$ is also referred to as a rough asymptotic or logarithmic asymptotic. More discussion on LDP will be presented in Section 8.

In Section 2, we introduce various geometric objects that are associated with an SRBM. For an SRBM that has a stationary distribution, we classify it into one of the three categories, I, II and III, based on some properties of these geometric objects. The characterization of the convergence domain $\mathcal{D}$ is stated in Theorem 2.1. The domain has a geometric description that uses the fixed point equations (2.8) and (2.9). Theorem 2.2 states exact asymptotic results for SRBMs in Category I, and Theorem 2.3 states exact asymptotic results for SRBMs in Category II. The results and proofs for Category III are omitted because it is symmetric to Category II. Section 3 gives a constructive procedure to solve the fixed point equations. This procedure is critical for us to iteratively identify parts of the convergence domain. Section 4 studies a key relationship among moment generating functions. This relationship and 
the iterative procedure in Section 3 allow us to identify the extreme points of the convergence domain $\mathcal{D}$ in Section 5. Section 6 presents some complex analysis preliminaries to the proofs of our main results. Section 7 devotes to the proofs of Theorems 2.1-2.3. Section 8 presents some concluding remarks.

2. Geometric properties and the main results. In this paper we consider a two-dimensional $\operatorname{SRBM} Z$ with data $(\Sigma, \mu, R)$. Setting

$$
R=\left(\begin{array}{ll}
r_{11} & r_{12} \\
r_{21} & r_{22}
\end{array}\right)
$$

throughout the paper except for Lemma 2.1, we assume that $R$ is a $\mathcal{P}$-matrix and $(R, \mu)$ satisfy (1.5); namely,

$$
\begin{aligned}
& r_{11}>0, \quad r_{22}>0, \quad r_{11} r_{22}-r_{12} r_{21}>0, \\
& r_{22} \mu_{1}-r_{12} \mu_{2}<0, \quad \text { and } \quad r_{11} \mu_{2}-r_{21} \mu_{1}<0 .
\end{aligned}
$$

A $\mathcal{P}$-matrix is a completely- $\mathcal{S}$ matrix; see [2]. Thus, it follows from [29] that, under condition (2.1), the SRBM exists and is unique in distribution. Together, conditions (2.1) and (2.2) are necessary and sufficient for the twodimensional SRBM to have a stationary distribution $[15,11]$. When it exists, the stationary distribution is unique. As before, we use $Z(\infty)$ to denote a two-dimensional random vector that has the stationary distribution.

As discussed in Section 1, the convergence domain (1) of the two-dimensional moment generating function $\varphi(\theta)$ defined in (1.9) is of primary importance in determining the asymptotic tail of $\mathbb{P}\{\langle c, Z(\infty)\rangle \geq u\}$ as $u \rightarrow \infty$. It turns out that the moment generating function $\varphi(\theta)$ is closely related to two boundary moment generating functions that we now define. For that, we first introduce two boundary measures. It follows from Proposition 3 of [3] that each component of $\mathbb{E}_{\pi}(Y(1))$ is finite, where $\mathbb{E}_{\pi}(\cdot)$ denotes the conditional expectation given that $Z(0)$ follows the stationary distribution $\pi$. For a Borel set $A \subset \mathbb{R}_{+}^{2}$, define

$$
\nu_{i}(A)=\mathbb{E}_{\pi} \int_{0}^{1} 1_{\{Z(u) \in A\}} d Y_{i}(u), \quad i=1,2 .
$$

Clearly, $\nu_{i}$ defines a finite measure on $\mathbb{R}_{+}^{2}$, which has a support on boundary $\left\{x \in \mathbb{R}_{+}^{2}: x_{i}=0\right\}$.

Let $\varphi_{i}$ be the moment generating function for $\nu_{i}$; namely,

$$
\begin{aligned}
& \varphi_{1}\left(\theta_{2}\right)=\int_{\mathbb{R}_{+}^{2}} e^{\theta_{2} x_{2}} \nu_{1}(d x)=\mathbb{E}_{\pi} \int_{0}^{1} e^{\theta_{2} Z_{2}(u)} d Y_{1}(u), \\
& \varphi_{2}\left(\theta_{1}\right)=\int_{\mathbb{R}_{+}^{2}} e^{\theta_{1} x_{1}} \nu_{2}(d x)=\mathbb{E}_{\pi} \int_{0}^{1} e^{\theta_{1} Z_{1}(u)} d Y_{2}(u) .
\end{aligned}
$$




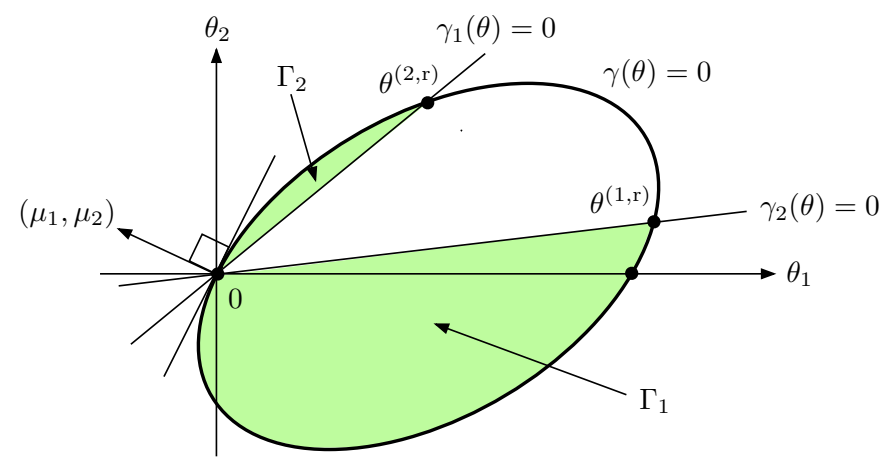

FIG 1. An ellipse for $\mu_{1}<0$ and $\mu_{2}>0$ : the ellipse $\gamma(\theta)=0$ intersects ray $\gamma_{1}(\theta)=0$ at $\theta^{(2, r)}$ and ray $\gamma_{2}(\theta)=0$ at $\theta^{(1, \mathrm{r})}$. Its tangent at the origin is orthogonal to $\mu=\left(\mu_{1}, \mu_{2}\right)$. Condition (2.2) means that the angle formed by vector $\mu$ and ray $\gamma_{k}(\theta)=0$ is more than $\pi / 2, k=1,2$. The shaded regions $\Gamma_{1}$ and $\Gamma_{2}$ are open sets defined in (2.7).

The pair $\varphi_{1}$ and $\varphi_{2}$ are referred to as the boundary moment generating functions.

We will prove the following facts in Proposition 4.1. For any $\theta=\left(\theta_{1}, \theta_{2}\right) \in$ $\mathbb{R}^{2}$ with $\varphi(\theta)<\infty$, we have that $\varphi_{1}\left(\theta_{2}\right)<\infty$ and $\varphi_{1}\left(\theta_{2}\right)<\infty$. Furthermore, the following key relationship among moment generating functions holds:

$$
\gamma(\theta) \varphi(\theta)=\gamma_{1}(\theta) \varphi_{1}\left(\theta_{2}\right)+\gamma_{2}(\theta) \varphi_{2}\left(\theta_{1}\right)
$$

where

$$
\begin{aligned}
& \gamma(\theta)=-\langle\theta, \mu\rangle-\frac{1}{2}\langle\theta, \Sigma \theta\rangle, \\
& \gamma_{1}(\theta)=r_{11} \theta_{1}+r_{21} \theta_{2}=\left\langle R^{1}, \theta\right\rangle, \quad \gamma_{2}(\theta)=r_{12} \theta_{1}+r_{22} \theta_{2}=\left\langle R^{2}, \theta\right\rangle,
\end{aligned}
$$

and $R^{k}$ is again the $k$ th column of $R$. Now we define some geometric objects that will play an important role for us to fully explore the key relationship (2.3) to characterize the convergence domain $\mathcal{D}$ defined in (1). Because $\Sigma$ is nonsingular, $\gamma(\theta)=0$ defines an ellipse $\partial \Gamma$ that passes through the origin. We use $\Gamma$ to denote the interior of the ellipse; namely,

$$
\Gamma=\left\{\theta \in \mathbb{R}^{2}: \gamma(\theta)>0\right\} .
$$

Define $\bar{\Gamma}$ to be the closure of $\Gamma$. From the definition of $\partial \Gamma, \mu=\left(\mu_{1}, \mu_{2}\right)$ is orthogonal to the tangent of the ellipse at the origin; see Figure 1. It is clear that $\gamma_{k}(\theta)=0$ is a line passing through the origin, $k=1,2$. For future purpose, we assign a direction for each line. For the line $\gamma_{1}(\theta)=0$, the direction is $\left(-r_{21}, r_{11}\right)$. For the line $\gamma_{2}(\theta)=0$, the direction is $\left(r_{22},-r_{12}\right)$. Each directional line is called a ray. We use the terms line and ray interchangeably. 
LEMmA 2.1. Assume that $r_{11}>0$ and $r_{22}>0$. Then, condition (2.2) holds true if and only if for each $k=1,2$ line $\gamma_{k}(\theta)=0$ intersects the ellipse $\gamma(\theta)=0$ at a point $\theta^{(3-k, \mathrm{r})}$ with $\theta_{1}^{(1, \mathrm{r})}>0$ and $\theta_{2}^{(2, \mathrm{r})}>0$. Furthermore, if (2.1) and (2.2) are satisfied, then either one of $\mu_{1}$ or $\mu_{2}$ is negative.

Proof. Let $v^{(1)}=\left(-r_{21}, r_{11}\right)$ and $v^{(2)}=\left(r_{22},-r_{12}\right)$. Then, $t v^{(1)}$ with variable $t>0$ represents ray 1 with the 2 nd coordinate to be positive, and $t v^{(2)}$ represents ray 2 with the 1 st coordinate to be positive.

Since vector $\mu \equiv\left(\mu_{1}, \mu_{2}\right)$ is orthogonal to the tangent of the ellipse $\gamma(\theta)=$ 0 at the origin and directed to the outside of ellipse, the conditions:

$$
\left\langle v^{(1)}, \mu\right\rangle<0, \quad\left\langle v^{(2)}, \mu\right\rangle<0
$$

are equivalent to that ray 1 intersects the ellipse at a point $\theta$ with $\theta_{2}>0$ and ray 2 intersects the ellipse at a point $\theta$ with $\theta_{1}>0$. Clearly, (2.4) is identical with (2.2). Thus, the first claim is proved.

Suppose both of $\mu_{1}$ and $\mu_{2}$ are nonnegative under (2.1) and (2.2). Then, both of $r_{12}$ and $r_{21}$ must be positive by (2.2). Multiplying the left and the right inequalities of (2.2) by $r_{11}$ and $r_{12}$, respectively, and then adding them together, we have

$$
\left(r_{22} r_{11}-r_{12} r_{21}\right) \mu_{1}<0 \text {. }
$$

But this is impossible because of (2.1). Hence, the second claim is proved.

We use $\theta^{(1, \mathrm{r})} \neq 0$ to denote the intersecting point of the ray $\gamma_{2}(\theta)=0$ and the ellipse $\gamma(\theta)=0$, and similarly use $\theta^{(2, \mathrm{r})} \neq 0$ to denote the intersecting point of the ray $\gamma_{1}(\theta)=0$ and the ellipse $\gamma(\theta)=0$. Here $r$ is mnemonic for ray. The unconventional index scheme for $\theta^{(k, \mathrm{r})}$ will be made clear in the next lemma: it derives from the fact that $\theta^{(1, r)}$ is close to the $\theta_{1}$ axis and $\theta^{(2, \mathrm{r})}$ is close to the $\theta_{2}$ axis.

Lemma 2.2. Let $\beta_{k}^{(\mathrm{r})} \in[-\pi, \pi]$ be the angle of ray $\gamma_{k}(\theta)=0$, measured counter clockwise starting from the $\theta_{1}$ axis. Then,

$$
-\frac{\pi}{2}<\beta_{2}^{(\mathrm{r})}<-\frac{\pi}{2}, \quad 0<\beta_{1}^{(\mathrm{r})}<\pi
$$

and

$$
\beta_{2}^{(\mathrm{r})}<\beta_{1}^{(\mathrm{r})}
$$

that is, ray $\gamma_{1}(\theta)=0$ is "above" ray $\gamma_{2}(\theta)=0$. 
Proof. Condition (2.5) is immediate from Lemma 2.1 because $r_{11}>0$ and $r_{22}>0$ are always assumed. If $\beta_{2}^{(\mathrm{r})} \in(-\pi / 2,0]$ or $\beta_{1}^{(\mathrm{r})} \in[\pi / 2, \pi)$, clearly (2.6) holds. Now we assume that $0<\beta_{1}^{(\mathrm{r})}, \beta_{2}^{(\mathrm{r})}<\pi / 2$. Because $R^{1}=$ $\left(r_{11}, r_{21}\right)^{\prime}$ is orthogonal to $\gamma_{1}(\theta)=0$, we have $r_{21}<0$. Similarly, we have $r_{12}<0$. Thus line $\gamma_{1}(\theta)=0$ has slope $-r_{11} / r_{21}$ and line $\gamma_{2}(\theta)$ has slope $-r_{12} / r_{22}$. Condition (2.1) implies that $r_{11} r_{22}>r_{12} r_{21}$ or

$$
-\frac{r_{11}}{r_{21}}>-\frac{r_{12}}{r_{22}}
$$

which implies (2.6).

Define open sets

$$
\begin{aligned}
& \Gamma_{1}=\left\{\theta \in \mathbb{R}^{2}: \gamma(\theta)>0, \gamma_{2}(\theta)<0\right\}, \\
& \Gamma_{2}=\left\{\theta \in \mathbb{R}^{2}: \gamma(\theta)>0, \gamma_{1}(\theta)<0\right\} .
\end{aligned}
$$

Clearly, they are nonempty. To abuse notation slightly, we define

$$
\partial \Gamma_{1}=\left\{\theta \in \partial \Gamma: \gamma_{2}(\theta) \leq 0\right\}, \quad \partial \Gamma_{2}=\left\{\theta \in \partial \Gamma: \gamma_{1}(\theta) \leq 0\right\} .
$$

Then $\partial \Gamma_{1}$ is the portion of boundary $\partial \Gamma$ that is below line $\gamma_{2}(\theta)=0$. Similarly, $\partial \Gamma_{2}$ is the portion of boundary $\partial \Gamma$ that is above line $\gamma_{1}(\theta)=0$.

The following pair of fixed points $\left(\tau_{1}, \tau_{2}\right)$ plays a critical role in this paper:

$$
\begin{aligned}
& \tau_{1}=\max \left\{\theta_{1}:\left(\theta_{1}, \theta_{2}\right) \in \partial \Gamma_{1}, \theta_{2} \leq \tau_{2}\right\}, \\
& \tau_{2}=\max \left\{\theta_{2}:\left(\theta_{1}, \theta_{2}\right) \in \partial \Gamma_{2}, \theta_{1} \leq \tau_{1}\right\} .
\end{aligned}
$$

To characterize the solution $\left(\tau_{1}, \tau_{2}\right)$ to the fixed point equations $(2.8)$ and (2.9), we classify the SRBM data $(\Sigma, \mu, R)$ into three categories. For this, define $\theta^{(1, \Gamma)}=\operatorname{argmax}_{\left(\theta_{1}, \theta_{2}\right) \in \partial \Gamma_{1}} \theta_{1}$ to be the right-most point on $\partial \Gamma_{1}$ and $\theta^{(2, \Gamma)}=\operatorname{argmax}_{\left(\theta_{1}, \theta_{2}\right) \in \partial \Gamma_{1}} \theta_{2}$ to be the highest point on $\partial \Gamma_{2}$. One can verify that

$\theta^{(1, \Gamma)}=\left\{\begin{array}{ll}\theta^{(1, \mathrm{r})} & \text { if } \theta^{(1, \max )} \notin \partial \Gamma_{1}, \\ \theta^{(1, \max )} & \text { if } \theta^{(1, \max )} \in \partial \Gamma_{1},\end{array} \quad \theta^{(2, \Gamma)}= \begin{cases}\theta^{(2, \mathrm{r})} & \text { if } \theta^{(2, \max )} \notin \partial \Gamma_{2}, \\ \theta^{(2, \max )} & \text { if } \theta^{(2, \max )} \in \partial \Gamma_{2},\end{cases}\right.$

where

$$
\theta^{(1, \max )}=\operatorname{argmax}_{\left(\theta, \theta_{2}\right) \in \partial \Gamma} \theta_{1} \quad \text { and } \quad \theta^{(2, \max )}=\operatorname{argmax}_{\left(\theta, \theta_{2}\right) \in \partial \Gamma} \theta_{2}
$$

are the right-most point and the highest point, respectively, on $\partial \Gamma$. Depending on the relative positions of $\theta^{(1, \Gamma)}$ and $\theta^{(2, \Gamma)}$, we introduce the following three categories (see [24]): 

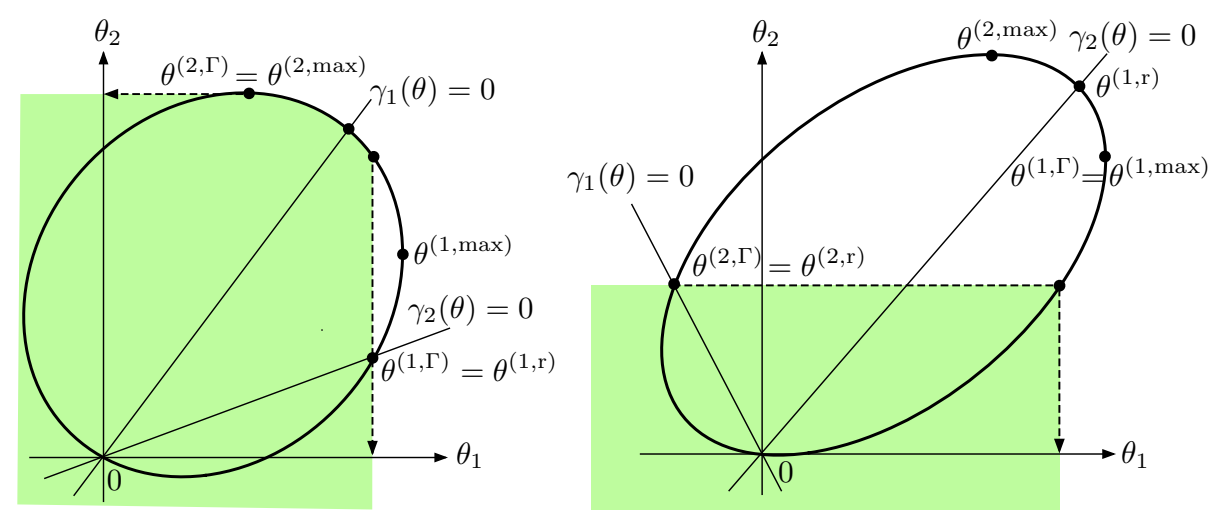

FIG 2. Convergence domains for Category I and Category II
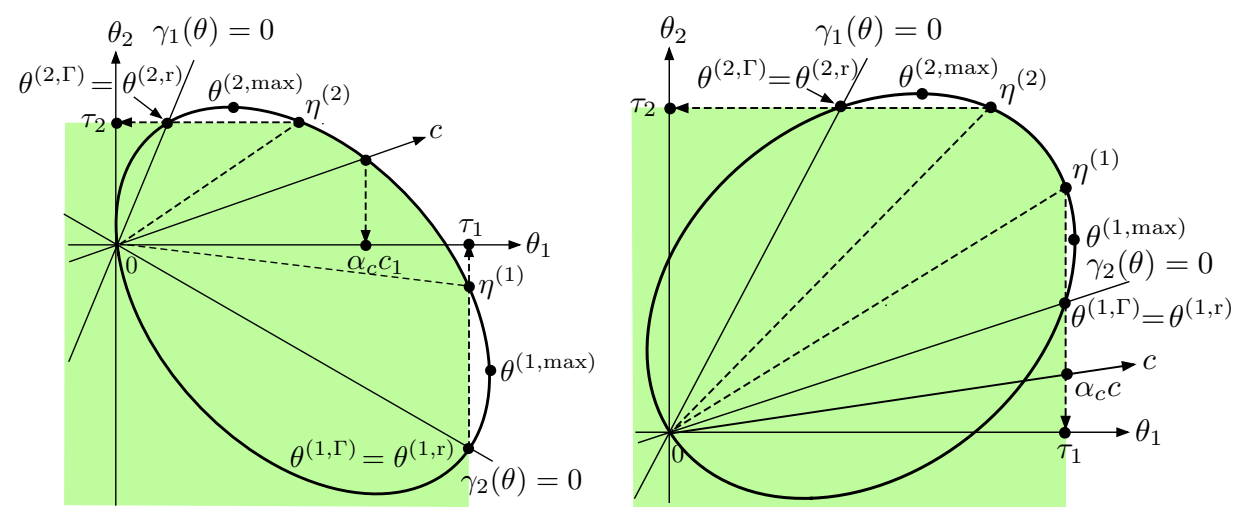

FIG 3. Two cases in Category I when $\tau_{1}<\theta_{1}^{(1, \max )}$

Category I: $\quad \theta_{1}^{(2, \Gamma)}<\theta_{1}^{(1, \Gamma)}$ and $\theta_{2}^{(1, \Gamma)}<\theta_{2}^{(2, \Gamma)}$,

Category II: $\quad \theta^{(2, \Gamma)} \leq \theta^{(1, \Gamma)}$,

Category III: $\quad \theta^{(1, \Gamma)} \leq \theta^{(2, \Gamma)}$.

Figure 2 illustrates Categories I and II. Because $\theta^{(1, \Gamma)}=\theta^{(2, \Gamma)}$ cannot happen, these three categories are mutually exclusive. We note that it is impossible to have the case where $\theta_{1}^{(2, \Gamma)}>\theta_{1}^{(1, \Gamma)}$ and $\theta_{2}^{(1, \Gamma)}>\theta_{2}^{(2, \Gamma)}$. This fact is proved in Lemma B.1 of Appendix B. Thus, these three categories indeed cover all SRBM data $(\Sigma, \mu, R)$. The following lemma characterizes the fixed point solution $\tau=\left(\tau_{1}, \tau_{2}\right)$; see Figures 3-6 for illustration of the characterization. This important lemma will be proved in Section 3 . 

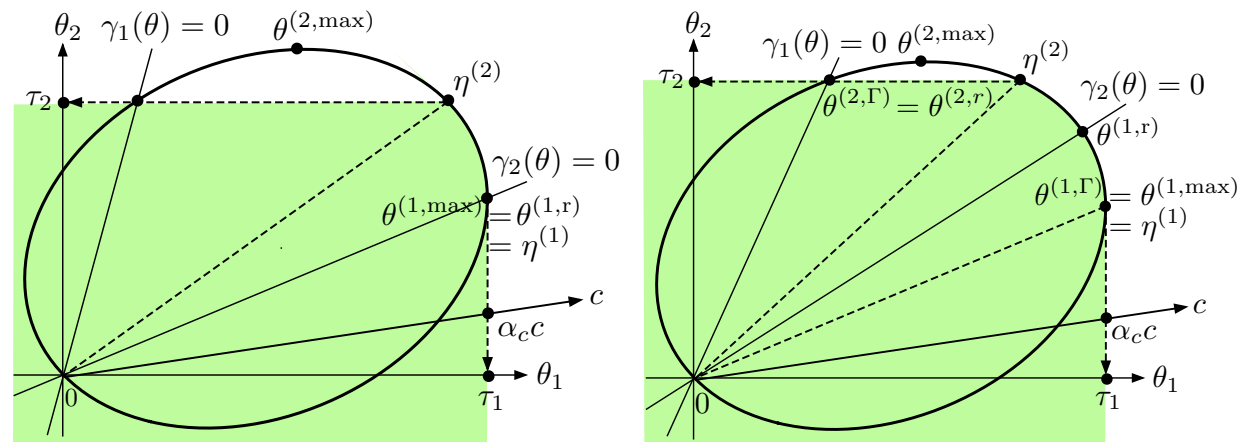

FIG 4. Two cases in Category I when $\tau_{1}=\theta_{1}^{(1, \max )}$

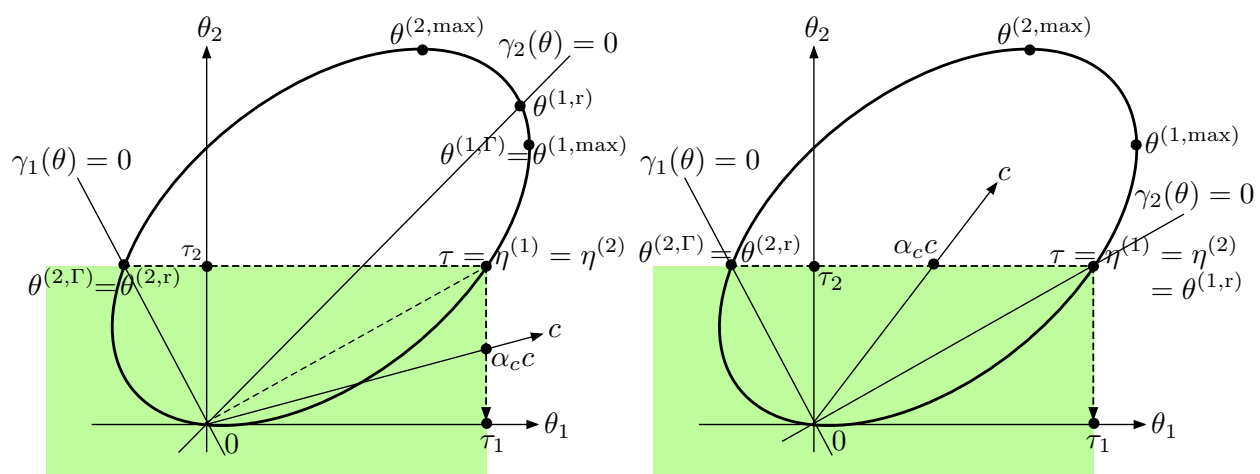

FIG 5. Two cases in Category II when $\tau_{1}<\theta_{1}^{(1, \max )}$

Lemma 2.3. There exists a unique solution $\tau=\left(\tau_{1}, \tau_{2}\right)$ to the fixed point equation (2.8) and (2.9). The solution $\tau$ is given by

$$
\tau= \begin{cases}\left(\theta_{1}^{(1, \Gamma)}, \theta_{2}^{(2, \Gamma)}\right) & \text { for Category I, } \\ \left(\bar{f}_{1}\left(\theta_{2}^{(2, \mathrm{r})}\right), \theta_{2}^{(2, \mathrm{r})}\right) & \text { for Category II, } \\ \left(\theta_{1}^{(1, \mathrm{r})}, \bar{f}_{2}\left(\theta_{1}^{(1, \mathrm{r})}\right)\right) & \text { for Category III. }\end{cases}
$$

Here, $\bar{f}_{2}$ is the function that represents the upper half of the ellipse $\partial \Gamma$, and similarly $\bar{f}_{1}$ is the function that represents the right half of the ellipse $\partial \Gamma$. For future purposes, we also let $f_{2}$ be the function that represents the lower half of the ellipse $\partial \Gamma$ and $\underline{f}_{1}$ be the function that represents the left half of the ellipse $\partial \Gamma$. Explicit expression for $\underline{f}_{2}$ is given in (6.2) in Section 6 . Expressions for other functions are given similarly. 

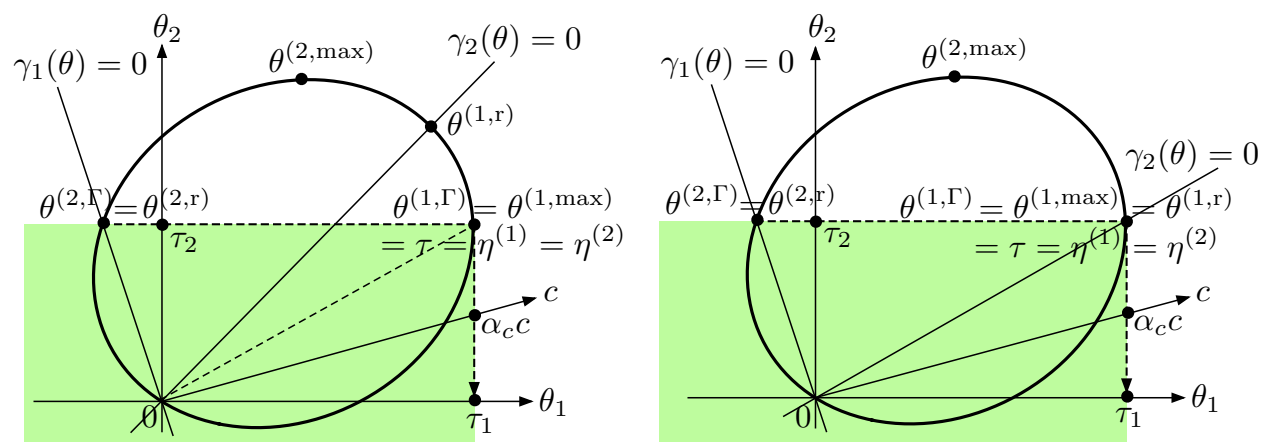

FIG 6. Two cases in Category II when $\tau_{1}=\theta_{1}^{(1, \max )}$

Setting

$$
\Gamma_{\max }=\left\{\theta \in \mathbb{R}^{2}: \theta<\tilde{\theta} \text { for some } \tilde{\theta} \text { such that } \gamma(\tilde{\theta})>0\right\},
$$

we have the following theorem characterizing of the convergence domain $\mathcal{D}$.

Theorem 2.1. Assume that conditions (2.1) and (2.2) hold. Then,

$$
\mathcal{D}=\Gamma_{\max }^{(\tau)} \equiv \Gamma_{\max } \cap\left\{\theta=\left(\theta_{1}, \theta_{2}\right) \in \mathbb{R}^{2}: \theta_{1}<\tau_{1} \text { and } \theta_{2}<\tau_{2}\right\} .
$$

It follows from Theorem 2.1 that when $\tau \in \bar{\Gamma}$, the convergence domain $\mathcal{D}$ is an (infinite) rectangle that has two pieces of boundary: $\theta_{1}=\tau_{1}$ and $\theta_{2}=\tau_{2}$. When $\tau$ is outside $\bar{\Gamma}$, the boundary $\partial \mathcal{D}$ consists of three pieces (Figures 3-4); they are $\theta_{1}=\tau_{1}, \theta_{2}=\tau_{2}$, and the part of the ellipse $\partial \Gamma$ between two points $\eta^{(1)}$ and $\eta^{(2)}$, where

$$
\eta^{(1)}=\left(\tau_{1}, \bar{f}_{2}\left(\tau_{1}\right)\right) \text { and } \eta^{(2)}=\left(\bar{f}_{1}\left(\tau_{2}\right), \tau_{2}\right) .
$$

For Categories II and III, $\tau \in \partial \Gamma$ must hold (Figures 5-6). It is possible that $\tau \in \Gamma$ for Category I (see Figure 10 in Section 6 for an example). As a convention, when $\tau \in \bar{\Gamma}$, we set

$$
\eta^{(1)}=\eta^{(2)}=\tau
$$

We now give the tail probability asymptotic of the convex combination

$$
c_{1} Z_{1}(\infty)+c_{2} Z_{2}(\infty)=\langle c, Z(\infty)\rangle
$$

for each direction vector $c \in \mathbb{R}_{+}^{2}$. For a point $x \neq 0$ in $\mathbb{R}^{2}$, by "line $x$ " we simply mean the line passing through the origin and $x$. Recall that $\alpha_{c}$ is 
used to denote the exponent in the exact asymptotic (1.6). Following the discussion in Section 1 and (1), $\alpha_{c}$ should be given by

$$
\alpha_{c}=\alpha>0 \text { such that } \alpha c \in \partial \mathcal{D} \cap \mathbb{R}_{+}^{2} .
$$

Indeed, throughout this paper, $\alpha_{c}$ is defined through (2.13). To compute $\alpha_{c}$, the intersection of the line $c$ with $\partial \mathcal{D}$ is important. In particular, it is helpful to see in which part of the boundary $\partial \mathcal{D} \cap \mathbb{R}_{+}^{2}$ this intersection is located. For this, let $\beta_{k}$ be the angle of line $\eta^{(k)}$, measured counter clockwise starting from $\theta_{1}$ axis, and let $\beta$ be the angle of line $c$. Point $c$ is said to be below line $\eta^{(k)}$ if $\beta<\beta_{k}$, and above line $\eta^{(k)}$ if $\beta>\beta_{k}$. To give an analytic expression for $\alpha_{c}$, let $z_{c}$ be the nonzero solution of $\gamma(z c)=0$. Then, $\alpha_{c}$ of (2.13) is given by

$$
\alpha_{c}= \begin{cases}\frac{\tau_{1}}{c_{1}}, & 0 \leq \beta<\beta_{1} \\ z_{c}, & \beta_{1} \leq \beta \leq \beta_{2} \\ \frac{\tau_{2}}{c_{2}}, & \beta_{2}<\beta \leq \frac{1}{2} \pi\end{cases}
$$

We first consider Category I. Recall the definition of $\eta^{(1)}$ and $\eta^{(2)}$ in (2.11) and (2.12). By Lemma 2.3, condition $\eta^{(1)} \neq \theta^{(1, \max )}$ is equivalent to condition $\tau_{1}<\theta_{1}^{(1, \max )}$, which is further equivalent to $\theta^{(1, \max )} \notin \partial \Gamma_{1}$.

THEOREM 2.2. Assume that conditions (2.1) and (2.2) hold and that the $S R B M$ data is in Category I. Let $c \in \mathbb{R}_{+}^{2}$ be a direction. Then, $\mathbb{P}(\langle c, Z(\infty)\rangle>$ $x)$ has the exact asymptotic $b f_{c}(x)$ with some constant $b>0$ and $f_{c}(x)$ being given below.

(a) When $c$ is below line $\eta^{(1)}$, i.e., $0 \leq \beta<\beta_{1}$,

$$
f_{c}(x)= \begin{cases}e^{-\alpha_{c} x} & \text { if } \eta^{(1)} \neq \theta^{(1, \max )}, \\ x^{-1 / 2} e^{-\alpha_{c} x} & \text { if } \eta^{(1)}=\theta^{(1, \max )}=\theta^{(1, \mathrm{r})} \\ x^{-3 / 2} e^{-\alpha_{c} x} & \text { if } \eta^{(1)}=\theta^{(1, \max )} \neq \theta^{(1, \mathrm{r})} .\end{cases}
$$

(b1) When $c$ is on line $\eta^{(1)} \neq \eta^{(2)}$, i.e., $\beta=\beta_{1}<\beta_{2}$,

$$
f_{c}(x)= \begin{cases}x e^{-\alpha_{c} x}, & \text { if } \eta^{(1)} \neq \theta^{(1, \max )} \\ e^{-\alpha_{c} x}, & \text { if } \eta^{(1)}=\theta^{(1, \max )}=\theta^{(1, \mathrm{r})}, \\ x^{-1 / 2} e^{-\alpha_{c} x}, & \text { if } \eta^{(1)}=\theta^{(1, \max )} \neq \theta^{(1, \mathrm{r})} .\end{cases}
$$

(b2) When $c$ is on line $\eta^{(1)}=\eta^{(2)}$, i.e., $\beta=\beta_{1}=\beta_{2}$,

$$
f_{c}(x)= \begin{cases}x e^{-\alpha_{c} x}, & \text { if } \tau \in \partial \Gamma, \\ e^{-\alpha_{c} x}, & \text { if } \tau \in \text { the interior of } \Gamma .\end{cases}
$$


(b3) When $c$ is above line $\eta^{(1)}$ and below line $\eta^{(2)}$, i.e., $\beta_{1}<\beta<\beta_{2}$,

$$
f_{c}(x)=e^{-\alpha_{c} x}
$$

(b4) When $c$ is on line $\eta^{(2)}$, the case is symmetric to (b1).

(c) When $c$ is above line $\eta^{(2)}$, the case is symmetric to (a).

REMARK 2.1. $\eta^{(1)}$ can not be above $\eta^{(2)}$ by their definitions. If $\beta_{1}<0$, then case (a) can not occur. Similarly, if $\beta_{2}>\frac{\pi}{2}$, then case (c) can not occur.

For Categories II and III, we only consider Category II because of their symmetry. In Category II, $\tau_{2}=\theta_{2}^{(2, \mathrm{r})}, \tau_{1}=\bar{f}_{1}\left(\tau_{2}\right), \eta^{(1)}=\eta^{(2)}=\tau=\left(\tau_{1}, \tau_{2}\right)$.

TheOrem 2.3. Assume that conditions (2.1) and (2.2) hold and the $S R B M$ data is in Category II. Let $c \in \mathbb{R}_{+}^{2}$ be a direction. Then, $\mathbb{P}(\langle c, Z\rangle>x)$ has the exact asymptotic $b f_{c}(x)$ with some constant $b>0$ and $f_{c}(x)$ being given below.

(a) When $c$ is below line $\tau\left(=\eta^{(1)}\right)$, i.e., $0 \leq \beta<\beta_{1}$,

$$
f_{c}(x)= \begin{cases}e^{-\alpha_{c} x}, & \text { if } \tau \neq \theta^{(1, \mathrm{r})} \text { and } \tau \neq \theta^{(1, \max )} \\ & \text { or if } \tau=\theta^{(1, \max )}=\theta^{(1, \mathrm{r})}, \\ x e^{-\alpha_{c} x}, & \text { if } \tau=\theta^{(1, \mathrm{r})} \neq \theta^{(1, \max )}, \\ x^{-1 / 2} e^{-\alpha_{c} x}, & \text { if } \tau=\theta^{(1, \max )} \neq \theta^{(1, \mathrm{r})} .\end{cases}
$$

(b) When $c$ is on line $\tau$, i.e., $\beta=\beta_{1}$,

$$
f_{c}(x)=x e^{-\alpha_{c} x} .
$$

(c) When $c$ is above line $\tau$, i.e, $\beta_{1}<\beta \leq \pi / 2$,

$$
f_{c}(x)=e^{-\alpha_{c} x}
$$

Theorem 2.1 will be proved in Section 7.1. Theorem 2.2 will be proved in Section 7.2, and Theorem 2.3 will be proved in Section 7.3.

3. Solution to the fixed point equations. Lemma 2.3 in Section 2 is an important lemma that establishes the existence and uniqueness of the solution $\tau$ to the fixed point equations. In this section, we prove this lemma. We separate the proof into two lemmas that are given below.

LEMMA 3.1. If there is a solution $\tau=\left(\tau_{1}, \tau_{2}\right) \neq 0$ to fixed point equations (2.8) and (2.9), $\tau$ must be given by (2.10). 
Proof. Let $\tau=\left(\tau_{1}, \tau_{2}\right)$ be a fixed point satisfying (2.8) and (2.9). We now show that $\tau$ must be given by formula (2.10). In the following argument, the two figures in Figure 2 are helpful. It follows from (2.8), (2.9) and the definition of $\theta^{(k, \Gamma)}$ that $\tau_{k} \leq \theta_{k}^{(k, \Gamma)}$ for $k=1,2$. One can verify that $\left(\tau_{1}, \underline{f}_{2}\left(\tau_{1}\right)\right) \in \partial \Gamma_{1}$ and $\left(\underline{f}_{1}\left(\tau_{2}\right), \tau_{2}\right) \in \partial \Gamma_{2}$. Thus, by (2.8) and (2.9) we have $\underline{f}_{2}\left(\tau_{1}\right) \leq \tau_{2}$ and $\underline{f}_{1}\left(\tau_{2}\right) \leq \tau_{1}$.

Assume first that $\tau_{1}<\theta_{1}^{(1, \Gamma)}$. We claim $\underline{f}_{2}\left(\tau_{1}\right)=\tau_{2}$. To see this, suppose that $\underline{f}_{2}\left(\tau_{1}\right)<\tau_{2}$. Then one can find a $\delta>0$ small enough such that $\tau_{1}+\delta<$ $\theta_{1}^{(1, \Gamma)}$ and $\underline{f}_{2}\left(\tau_{1}+\delta\right)<\tau_{2}$. The former inequality implies that $\left(\tau_{1}+\delta, \underline{f}_{2}\left(\tau_{1}+\right.\right.$ $\delta)) \in \partial \Gamma_{1}$. This, together with the fact that $\underline{f}_{2}\left(\tau_{1}+\delta\right)<\tau_{2}$, contradicts with the definition of $\tau_{1}$ in (2.8). Therefore, we have proved that $\tau_{1}<\theta_{1}^{(1, \Gamma)}$ implies that $\underline{f}_{2}\left(\tau_{1}\right)=\tau_{2}$. Similarly, we can prove that $\tau_{2}<\theta_{2}^{(2, \Gamma)}$ implies that $\underline{f}_{1}\left(\tau_{2}\right)=\tau_{1}$. Now we claim that $\tau_{1}<\theta_{1}^{(1, \Gamma)}$ and $\tau_{2}<\theta_{2}^{(2, \Gamma)}$ cannot happen simultaneously. Otherwise, $\underline{f}_{1}\left(\tau_{2}\right)=\tau_{1}$ and $\underline{f}_{2}\left(\tau_{1}\right)=\tau_{2}$, and therefore

$$
\left(\underline{f}_{1}\left(\tau_{2}\right), \tau_{2}\right)=\left(\tau_{1}, \underline{f}_{2}\left(\tau_{1}\right)\right) \in \partial \Gamma_{1} \cap \partial \Gamma_{2} \cap \mathbb{R}_{+}^{2} .
$$

However, this is possible only for $\tau_{1}=\tau_{2}=0$ because $\bar{\Gamma}_{1} \cap \bar{\Gamma}_{2} \cap \mathbb{R}_{+}^{2}=\{0\}$ by Lemma 2.2. Now $\tau=0$ contradicts the assumption that $\tau \neq 0$ and thus the claim is proved.

Hence, $\tau_{1}<\theta_{1}^{(1, \Gamma)}$ implies that $\underline{f}_{2}\left(\tau_{1}\right)=\tau_{2}=\theta_{2}^{(2, \Gamma)}$. In this case, it is impossible to have $\theta_{2}^{(1, \Gamma)} \leq \theta_{2}^{(2, \Gamma)}$ since the latter implies $\tau_{1}=\theta_{1}^{(1, \Gamma)}$ by (2.8). Thus, we have $\theta_{2}^{(1, \Gamma)}>\theta_{2}^{(2, \Gamma)}$. On the other hand, $\tau_{2}=\theta_{2}^{(2, \Gamma)}$ implies $\underline{f}_{1}\left(\tau_{2}\right)=\theta_{1}^{(2, \Gamma)}$, so

$$
\theta_{1}^{(2, \Gamma)}=\underline{f}_{1}\left(\tau_{2}\right) \leq \tau_{1}<\theta_{1}^{(1, \Gamma)} .
$$

Hence, we have $\theta^{(2, \Gamma)}<\theta^{(1, \Gamma)}$. Thus, $\theta_{2}^{(2, \Gamma)}<\theta_{2}^{(1, \Gamma)} \leq \theta_{2}^{(2, \max )}$, and therefore $\theta_{2}^{(2, \Gamma)}$ must be $\theta_{2}^{(2, \mathrm{r})}$. Thus, $\tau_{2}=\theta_{2}^{(2, \mathrm{r})}$. Because, $\left(\tau_{1}, \tau_{2}\right) \in \partial \Gamma, \tau_{1}$ must be either $\underline{f}_{1}\left(\tau_{2}\right)$ or $\bar{f}_{1}\left(\tau_{2}\right)$. The former is impossible because it leads to (3.1). Therefore, we have proved that if $\tau_{1}<\theta_{1}^{(1, \Gamma)}$, we must have Category II; furthermore, we have $\tau_{2}=\theta_{2}^{(2, \mathrm{r})}$ and $\tau_{1}=\bar{f}_{1}\left(\tau_{2}\right)$. Thus, we have proved that when $\tau_{1}<\theta_{1}^{(1, \Gamma)}, \tau$ is given in (2.10) for Category II. Similarly, we can prove that when $\tau_{2}<\theta_{2}^{(2, \Gamma)}, \tau$ is given in (2.10) for Category III.

The remaining possibility is that $\tau_{1}=\theta_{1}^{(1, \Gamma)}$ and $\tau_{2}=\theta_{2}^{(2, \Gamma)}$. We have $\theta_{1}^{(2, \Gamma)}=\underline{f}_{1}\left(\tau_{2}\right) \leq \tau_{1}=\theta_{1}^{(1, \Gamma)}$ and $\theta_{2}^{(1, \Gamma)}=\underline{f}_{2}\left(\tau_{1}\right) \leq \tau_{2}=\theta_{2}^{(2, \Gamma)}$. Since we can not simultaneously have $\underline{f}_{1}\left(\tau_{2}\right)=\tau_{1}$ and $\underline{f}_{2}\left(\tau_{1}\right)=\tau_{2}$, either $\theta_{1}^{(2, \Gamma)}<\tau_{1}$ or 
$\theta_{2}^{(1, \Gamma)}<\tau_{2}$ holds. Thus $\tau$ is given in (2.10) for Category I if $\theta_{1}^{(2, \Gamma)}<\theta_{1}^{(1, \Gamma)}$ and $\theta_{2}^{(1, \Gamma)}<\theta_{2}^{(2, \Gamma)}$. Otherwise, $\tau$ is given in (2.10) for Category II or Category III if $\theta_{2}^{(1, \Gamma)}=\theta_{2}^{(2, \Gamma)}$ or $\theta_{1}^{(2, \Gamma)}=\theta_{1}^{(1, \Gamma)}$ holds, respectively.

The following lemma is similar to Lemma 3.4 of [17] (see also Corollary 4.1 of [24]), but a proof in our setting is simpler.

Lemma 3.2. There is a nonnegative solution $\tau=\left(\tau_{1}, \tau_{2}\right) \neq 0$ to fixed point equations (2.8) and (2.9).

Proof. Let $\tau^{(0)}=(0,0)$. For $n \geq 1$ define $\tau_{1}^{(n)}$ and $\tau_{2}^{(n)}$ recursively via

$$
\begin{aligned}
& \tau_{1}^{(n)}=\max \left\{\theta_{1}:\left(\theta_{1}, \theta_{2}\right) \in \partial \Gamma_{1}, \quad \theta_{2} \leq \tau_{2}^{(n-1)}\right\}, \\
& \tau_{2}^{(n)}=\max \left\{\theta_{2}:\left(\theta_{1}, \theta_{2}\right) \in \partial \Gamma_{2}, \quad \theta_{1} \leq \tau_{1}^{(n-1)}\right\} .
\end{aligned}
$$

One can check that (3.2) and (3.3) are equivalent to the following

$$
\begin{aligned}
& \tau_{1}^{(n)}=\max \left\{\theta_{1} \in\left[0, \theta_{1}^{(1, \Gamma)}\right], \quad \underline{f}_{2}\left(\theta_{1}\right) \leq \tau_{2}^{(n-1)}\right\}, \\
& \tau_{2}^{(n)}=\max \left\{\theta_{2} \in\left[0, \theta_{2}^{(2, \Gamma)}\right] \quad \underline{f}_{1}\left(\theta_{2}\right) \leq \tau_{1}^{(n-1)}\right\} .
\end{aligned}
$$

We now use induction to show that

$$
\begin{aligned}
& \tau_{k}^{(n-1)} \leq \tau_{k}^{(n)} \leq \theta_{k}^{(k, \Gamma,)} \quad k=1,2 \\
& \underline{f}_{2}\left(\tau_{1}^{(n)}\right) \leq \tau_{2}^{(n)}, \quad \text { and } \quad \underline{f}_{1}\left(\tau_{2}^{(n)}\right) \leq \tau_{1}^{(n)} .
\end{aligned}
$$

For $n=1$, clearly (3.6) holds. By the definition of $\tau_{1}^{(1)}$, we have $f_{2}\left(\tau_{1}^{(1)}\right) \leq$ $0 \leq \tau_{1}^{(1)}$. Similarly, we have $f_{1}\left(\tau_{2}^{(1)}\right) \leq 0 \leq \tau_{2}^{(1)}$. Thus, (3.7) holds for $n=1$. Suppose that (3.6) and (3.7) hold for $n$. We would like to show that (3.6) and (3.7) hold for $n+1$. Because $0 \leq \tau_{1}^{(n)} \leq \theta_{1}^{(\Gamma, 1)}$ and $\underline{f}_{2}\left(\tau_{1}^{(n)}\right) \leq \tau_{2}^{(n)}$ by the induction assumption, it follows from definition (3.4) that $\tau_{1}^{(n)} \leq$ $\tau_{1}^{(n+1)} \leq \theta_{1}^{(1, \Gamma)}$. Similarly, we have $\tau_{2}^{(n)} \leq \tau_{2}^{(n+1)} \leq \theta_{2}^{(2, \Gamma)}$. Thus, we have proved (3.6) for $n+1$. Now, by the definition of $\tau_{1}^{(n+1)}$ and $\tau_{2}^{(n+1)}$, we have $\underline{f}_{2}\left(\tau_{1}^{(n+1)}\right) \leq \tau_{2}^{(n)} \leq \tau_{2}^{(n+1)}$ and $\underline{f}_{1}\left(\tau_{2}^{(n+1)}\right) \leq \tau_{1}^{(n)} \leq \tau_{1}^{(n+1)}$, proving (3.7) for $n+1$.

By (3.6), the two sequences $\left\{\tau_{1}^{(n)}: n \geq 0\right\}$ and $\left\{\tau_{1}^{(n)}: n \geq 0\right\}$ are nondecreasing and bounded. Thus

$$
\tau_{k}=\lim _{n \rightarrow \infty} \tau_{k}^{(n)}
$$


is well defined. We now prove that

$$
\tau_{1}=\max \left\{\theta_{1} \in\left[0, \theta_{1}^{(1, \Gamma)}\right], \quad \underline{f}_{2}\left(\theta_{1}\right) \leq \tau_{2}\right\} .
$$

Let $\tau_{1}^{*}=\max \left\{\theta_{1} \in\left[0, \theta_{1}^{(1, \Gamma)}\right], \quad \underline{f}_{2}\left(\theta_{1}\right) \leq \tau_{2}\right\}$. It suffices to prove that $\tau_{1}=\tau_{1}^{*}$. Since $\underline{f}_{2}\left(\theta_{1}\right)$ is decreasing in $\left[\theta_{1}^{(1, \min )}, \theta_{1}^{(2, \mathrm{~min})}\right]$ and increasing in $\left[\theta_{1}^{(2, \min )}, \theta_{1}^{(1, \max )}\right]$, we have

$$
\tau_{1}^{*}=\max \left\{\theta_{1} \in\left[\theta_{1}^{(2, \min )}, \theta_{1}^{(1, \Gamma)}\right], \quad \underline{f}_{2}\left(\theta_{1}\right) \leq \tau_{2}\right\} .
$$

If $\underline{f}_{2}\left(\tau_{1}\right)=\tau_{2}$, by the monotonicity of $\underline{f}_{2}, \tau_{1}^{*}=\tau_{1}$. Assume that $\underline{f}_{2}\left(\tau_{1}\right)<\tau_{2}$. By the continuity of $\underline{f}_{2}$, there exists an $N>0$ such that for $n \geq N$,

$$
\underline{f}_{2}\left(\tau_{1}^{(n)}\right)<\tau_{2}^{(n-1)}
$$

Since $\tau_{1}^{(n)}$ satisfies (3.4), we have $\tau_{1}^{(n)}=\theta_{1}^{(1, \Gamma)}$. Thus, $\underline{f}_{2}\left(\theta_{1}^{(1, \Gamma)}\right)<\tau_{2}^{(n-1)}$ for $n \geq N$. Letting $n \rightarrow \infty$, we have $\tau_{1}=\theta_{1}^{(1, \Gamma)}$ and $\underline{f}_{2}\left(\theta_{1}^{(1, \Gamma)}\right) \leq \tau_{2}$. The latter implies that $\tau_{1}^{*}=\theta_{1}^{(1, \Gamma)}$. Thus, we have $\tau_{1}=\tau_{1}^{*}$. We can prove similarly that

$$
\tau_{2}=\max \left\{\theta_{2} \in\left[0, \theta_{2}^{(2, \Gamma)}\right], \quad \underline{f}_{1}\left(\theta_{2}\right) \leq \tau_{1}\right\} .
$$

Therefore, we have proved that $\tau_{1}$ and $\tau_{2}$ satisfy (2.8) and (2.9).

It remains to show that $\tau=\left(\tau_{1}, \tau_{2}\right) \neq(0,0)$. For this, it suffices to prove that $\tau^{(1)} \neq(0,0)$. Recall that

$$
\begin{aligned}
& \tau_{1}^{(1)}=\max \left\{\theta_{1}:\left(\theta_{1}, \theta_{2}\right) \in \partial \Gamma_{1}, \quad \theta_{2} \leq 0\right\}, \\
& \tau_{2}^{(1)}=\max \left\{\theta_{2}:\left(\theta_{1}, \theta_{2}\right) \in \partial \Gamma_{2}, \quad \theta_{1} \leq 0\right\} .
\end{aligned}
$$

Since vector $\left(\mu_{1}, \mu_{2}\right)$ is orthogonal to the tangent of the ellipse $\partial \Gamma$ at the origin and at least one of $\mu_{1}$ and $\mu_{2}$ is negative because of Lemma 2.1, the ellipse $\partial \Gamma$ intersects at least one of the two regions $\left\{\left(\theta_{1}, \theta_{2}\right) \in \mathbb{R}^{2}: \theta_{1}<\right.$ $\left.0, \theta_{2}>0\right\}$ and $\left\{\left(\theta_{1}, \theta_{2}\right) \in \mathbb{R}^{2}: \theta_{1}>0, \theta_{2}<0\right\}$. This implies that at least one of $\tau_{1}^{(1)}$ and $\tau_{2}^{(1)}$ must be positive. This concludes $\tau^{(1)} \neq 0$.

4. A key relationship among generating functions. In this section, we prove the key relationship (2.3) among the moment generating functions. The following lemma proves the relationship under several sets of conditions. This lemma is the key to the proofs of Theorems 2.1-2.3. Recall that $\Gamma_{1}$ and $\Gamma_{2}$ are defined in (2.7). 
Lemma 4.1. Assume that conditions (2.1) and (2.2) hold. (a) For each $\theta \in \mathbb{R}^{2}$ with $\varphi(\theta)<\infty, \varphi_{1}\left(\theta_{2}\right)<\infty$ and $\varphi_{2}\left(\theta_{1}\right)<\infty$, the key relationship (2.3) holds. (b) For each $\theta \in \mathbb{R}^{2}, \varphi(\theta)<\infty$ implies that $\varphi_{1}\left(\theta_{2}\right)<\infty$ and $\varphi_{2}\left(\theta_{1}\right)<\infty$. (c) For each $\theta \in \Gamma, \varphi_{1}\left(\theta_{2}\right)<\infty$ and $\varphi_{2}\left(\theta_{1}\right)<\infty$ imply that $\varphi(\theta)<\infty$. (d) For each $\theta \in \Gamma_{1}, \varphi_{1}\left(\theta_{2}\right)<\infty$ implies that $\varphi(\theta)<\infty$. Similarly, for each $\theta \in \Gamma_{2}, \varphi_{2}\left(\theta_{1}\right)<\infty$ implies that $\varphi(\theta)<\infty$.

We will present the proof of this lemma later in this section. The tool for the proof is the basic adjoint relationship (4.1) below that governs the stationary distribution $\pi$ and the corresponding boundary measures $\nu_{1}$ and $\nu_{2}$. To state the basic adjoint relationship, let $C_{b}^{2}\left(\mathbb{R}_{+}^{2}\right)$ be the set of twice continuously differentiable functions $f$ on $\mathbb{R}_{+}^{2}$ such that $f$ and its first- and second-order derivatives are bounded. For each $f \in C_{b}^{2}\left(\mathbb{R}_{+}^{2}\right)$, define

$$
\begin{aligned}
& L f(x)=\frac{1}{2} \sum_{i=1}^{2} \sum_{j=1}^{2} \Sigma_{i j} \frac{\partial^{2} f}{\partial x_{i} \partial x_{j}}(x)+\sum_{i=1}^{2} \mu_{i} \frac{\partial f}{\partial x_{i}}(x), \\
& D_{1} f(x)=r_{11} \frac{\partial f}{\partial x_{1}}(x)+r_{21} \frac{\partial f}{\partial x_{2}}(x)=\left\langle R^{1}, \nabla f(x)\right\rangle, \\
& D_{2} f(x)=r_{12} \frac{\partial f}{\partial x_{1}}(x)+r_{22} \frac{\partial f}{\partial x_{2}}(x)=\left\langle R^{2}, \nabla f(x)\right\rangle .
\end{aligned}
$$

Then the basic adjoint relationship takes the following form:

$$
\int_{\mathbb{R}_{+}^{2}} L f(x) \pi(d x)+\sum_{i=1}^{2} \int_{\mathbb{R}_{+}^{2}} D_{i} f(x) \nu_{i}(d x)=0 \quad \text { for each } f \in C_{b}^{2}\left(\mathbb{R}_{+}^{2}\right) .
$$

The basic adjoint relationship (4.1) is now standard in the SRBM literature. It was first proved in [13] for an SRBM in $\mathbb{R}_{+}^{d}$ for any integer $d \geq 1$ when $R$ is an $\mathcal{M}$ matrix. Extension to a general SRBM, when its stationary distribution exists, can be found, for example, in [3].

Proof of Lemma 4.1. (a) Assume that $\varphi(\theta)<\infty, \varphi_{1}\left(\theta_{2}\right)<\infty$ and $\varphi_{2}\left(\theta_{1}\right)<\infty$. Define

$$
f\left(x_{1}, x_{2}\right)=e^{\theta_{1} x_{1}+\theta_{2} x_{2}} \quad \text { for } x \in \mathbb{R}_{+}^{2} .
$$

This $f$ is generally not in $C_{b}^{2}\left(\mathbb{R}_{+}^{2}\right)$, and thus (4.1) can not be applied directly. To overcome this difficulty, we construct a sequence of functions $\left\{f_{n}\right\} \subset$ $C_{b}^{2}\left(\mathbb{R}_{+}^{2}\right)$ to approximate $f$. To this end, for each positive integer $n$, define 
function $g_{n}$ as

$$
g_{n}(s)= \begin{cases}1, & s \leq n, \\ 1-\frac{1}{2}(s-n)^{2}, & n<s \leq n+1, \\ \frac{1}{2}(n+2-s)^{2}, & n+1<s \leq n+2, \\ 0, & s>n+2 .\end{cases}
$$

Then, $g_{n}$ is continuously differentiable on $\mathbb{R}$. We then define $h_{n}$ as

$$
h_{n}(u)= \begin{cases}\int_{0}^{u} g_{n}(s) d s, & u \geq 0 \\ u, & u<0 .\end{cases}
$$

Clearly, for each fixed $n, h_{n}(u)$ is twice continuously differentiable. Furthermore, for each $u \in \mathbb{R}_{+}, h_{n}(u)$ and $g_{n}(u)$ are monotone in $n$, and

$$
\lim _{n \rightarrow \infty} h_{n}(u)=u, \quad \lim _{n \rightarrow \infty} g_{n}(u)=1 .
$$

For each $n$, define

$$
f_{n}(x)=e^{h_{n}(\langle\theta, x\rangle)} .
$$

Because $h_{n}(u)$ is a constant for $u>n+2, f_{n} \in C_{b}^{2}\left(\mathbb{R}_{+}\right)$. Clearly $f_{n} \rightarrow f$ monotonously as $n \rightarrow \infty$. Plugging $f_{n}$ into (4.1), one has

$$
\begin{aligned}
& \frac{1}{2} \sum_{i=1}^{2} \sum_{j=1}^{2} \Sigma_{i j} \theta_{i} \theta_{j} \int_{\mathbb{R}_{+}^{2}}\left(g_{n}^{2}(\langle\theta, x\rangle)+g_{n}^{\prime}(\langle\theta, x\rangle)\right) f_{n}(x) \pi(d x) \\
& \quad+\sum_{i=1}^{2} \mu_{i} \theta_{i} \int_{\mathbb{R}_{+}^{2}} g_{n}(\langle\theta, x\rangle) f_{n}(x) \pi(d x) \\
& \quad+\left(r_{11} \theta_{1}+r_{21} \theta_{2}\right) \int_{\mathbb{R}_{+}^{2}} g_{n}\left(\theta_{2} x_{2}\right) f_{n}\left(0, x_{2}\right) \nu_{1}(d x) \\
& \quad+\left(r_{22} \theta_{2}+r_{12} \theta_{1}\right) \int_{\mathbb{R}_{+}^{2}} g_{n}\left(\theta_{1} x_{1}\right) f_{n}\left(x_{1}, 0\right) \nu_{2}(d x)=0 .
\end{aligned}
$$

For each $n$, one can verify that $-1 \leq g_{n}^{\prime}(s) \leq 0$ for all $s \in \mathbb{R}_{+}$and $f_{n}\left(x_{1}, x_{2}\right) \leq f\left(x_{1}, x_{2}\right)$ for all $\left(x_{1}, x_{2}\right) \in \mathbb{R}_{+}^{2}$. Because $\varphi(\theta)<\infty$ and $\lim _{n \rightarrow \infty} g_{n}^{\prime}(s)=0$ for each $s \in \mathbb{R}_{+}$, by dominated convergence theorem,

$$
\lim _{n \rightarrow \infty} \int_{\mathbb{R}_{+}^{2}} g_{n}^{\prime}(\langle\theta, x\rangle) f_{n}(x) \pi(d x)=0 .
$$

Taking limit on both sides of (4.3) as $n \rightarrow \infty$ and using monotone convergence theorem and (4.4), one has

$$
-\gamma(\theta) \varphi(\theta)+\gamma_{1}(\theta) \varphi_{1}\left(\theta_{2}\right)+\gamma_{2}(\theta) \varphi_{2}\left(\theta_{1}\right)=0,
$$

proving (2.3). 
(b) Assume $\varphi(\theta)<\infty$. We first show that $\varphi_{2}\left(\theta_{1}\right)<\infty$. If $\theta_{1} \leq 0$, the conclusion is trivial. Now we assume $\theta_{1}>0$. Without loss of generality, we assume that

$$
\theta_{2}<-\theta_{1} \frac{\left|r_{12}\right|}{r_{22}}
$$

From inequality (4.5), we have $\gamma_{2}(\theta)<0$ and $\varphi_{1}\left(\theta_{2}\right)<\infty$. Letting $n \rightarrow \infty$ in both sides of (4.3) as in the proof of part (a), we conclude the finiteness of $\varphi_{2}\left(\theta_{1}\right)$. The proof for $\varphi_{1}\left(\theta_{2}\right)<\infty$ is similar.

(c) Assume $\theta \in \Gamma, \varphi_{1}\left(\theta_{2}\right)<\infty$ and $\varphi_{2}\left(\theta_{1}\right)<\infty$. We would like to show that $\varphi(\theta)<\infty$. For this, we again use (4.3). Applying the facts that $0 \leq g_{n}(\langle\theta, x\rangle) \leq 1,\langle\theta, \Sigma \theta\rangle \geq 0$ and $g_{n}^{\prime}(\langle\theta, x\rangle) \leq 0$ to (4.3) yields

$$
\begin{aligned}
& \left(\frac{1}{2}\langle\theta, \Sigma \theta\rangle+\langle\mu, \theta\rangle\right) \int_{\mathbb{R}_{+}^{2}} g_{n}(\langle\theta, x\rangle) f_{n}(x) \pi(d x) \\
& \quad+\gamma_{1}(\theta) \int_{\mathbb{R}_{+}^{2}} g_{n}\left(\theta_{2} x_{2}\right) f_{n}\left(0, x_{2}\right) \nu_{1}(d x) \\
& \quad+\gamma_{2}(\theta) \int_{\mathbb{R}_{+}^{2}} g_{n}\left(\theta_{1} x_{1}\right) f_{n}\left(x_{1}, 0\right) \nu_{2}(d x) \geq 0 .
\end{aligned}
$$

Since $\frac{1}{2}\langle\theta, \Sigma \theta\rangle+\langle\mu, \theta\rangle=-\gamma(\theta)<0$, the monotone convergence theorem yields

$$
\gamma(\theta) \varphi(\theta) \leq \gamma_{1}(\theta) \varphi_{1}\left(\theta_{2}\right)+\gamma_{2}(\theta) \varphi_{2}\left(\theta_{1}\right)<\infty .
$$

This completes the proof since $\gamma(\theta)>0$.

(d) Assume $\theta \in \Gamma_{1}$ and $\varphi_{1}\left(\theta_{2}\right)<\infty$. We would like to show that $\varphi(\theta)<$ $\infty$. For this, we again use (4.3). Applying the facts that $\gamma_{2}(\theta)<0,0 \leq$ $g_{n}(\langle\theta, x\rangle) \leq 1,\langle\theta, \Sigma \theta\rangle \geq 0$ and $g_{n}^{\prime}(\langle\theta, x\rangle) \leq 0$ to (4.3) yields

$$
\begin{array}{r}
\left(\frac{1}{2}\langle\theta, \Sigma \theta\rangle+\langle\mu, \theta\rangle\right) \int_{\mathbb{R}_{+}^{2}} g_{n}(\langle\theta, x\rangle) f_{n}(x) \pi(d x) \\
\quad+\gamma_{1}(\theta) \int_{\mathbb{R}_{+}^{2}} g_{n}\left(\theta_{2} x_{2}\right) f_{n}\left(0, x_{2}\right) \nu_{1}(d x) \geq 0 .
\end{array}
$$

Again using the fact that $\frac{1}{2}\langle\theta, \Sigma \theta\rangle+\langle\mu, \theta\rangle=-\gamma(\theta)<0$ and the monotone convergence theorem, we have

$$
\gamma(\theta) \varphi(\theta) \leq \gamma_{1}(\theta) \varphi_{1}\left(\theta_{2}\right)<\infty .
$$

This completes the proof since $\gamma(\theta)>0$ and $\varphi\left(\theta_{2}\right)<\infty$. Similarly, for each $\theta \in \Gamma_{2}$ with $\varphi_{2}\left(\theta_{1}\right)<\infty$, we can prove $\varphi(\theta)<\infty$. 
5. Extreme points of the convergence domain. This section is a preliminary to the proof of Theorem 2.1. In this section, we identify extreme points of the convergence domain $\mathcal{D}$ defined in (1). For this, we establish two lemmas, Lemmas 5.1 and 5.2. A consequence of Lemma 5.1 and part (c) of Lemma 4.1 is that $\Gamma_{\max }^{(\tau)} \subset \mathcal{D}$, where $\Gamma_{\max }^{(\tau)}$ is defined in $(2.1)$. To prove Theorem 2.1, we need to show the converse $\mathcal{D} \subset \Gamma_{\text {max }}^{(\tau)}$. Lemma 5.2 shows a partial converse. We can not fully establish this converse in this section. To establish converse $\mathcal{D} \subset \Gamma_{\max }^{(\tau)}$, we need to use complex variable functions and their analytic extensions as discussed in Section 6.

Lemma 5.1. Condition $\theta_{1}<\tau_{1}$ implies $\varphi_{2}\left(\theta_{1}\right)<\infty$, and condition $\theta_{2}<$ $\tau_{2}$ implies $\varphi_{1}\left(\theta_{2}\right)<\infty$.

Proof. We use Lemma 4.1 to iteratively expand a confirmed region on which $\varphi(\theta)<\infty$. Recall two sequences $\left\{\tau_{1}^{(n)}: n \geq 0\right\}$ and $\left\{\tau_{2}^{(n)}: n \geq 0\right\}$ defined in the proof of Lemma 3.2 in Section 3. We use induction to prove that, for each $n \geq 0$, condition $\theta_{1}<\tau_{1}^{(n)}$ implies $\varphi_{2}\left(\theta_{1}\right)<\infty$, and condition $\tau_{2}<\tau_{2}^{(n)}$ implies $\varphi_{1}\left(\theta_{2}\right)<\infty$. Since $\tau_{1}^{(0)}=\tau_{2}^{(0)}=0$, the conclusion holds for $n=0$ because $\varphi_{k}(0)=\nu_{k}\left(\mathbb{R}_{+}^{2}\right)<\infty$. Assume that condition $\theta_{1}<\tau_{1}^{(n-1)}$ implies $\varphi_{2}\left(\theta_{1}\right)<\infty$, and condition $\theta_{2}<\tau_{2}^{(n-1)}$ implies $\varphi_{1}\left(\theta_{2}\right)<\infty$. We first assume $\theta_{2}<\tau_{2}^{(n)}$. If $\tau_{2}^{(n)}=0, \varphi_{1}\left(\theta_{2}\right)<0$ clearly holds. Assume that $\tau_{2}^{(n)}>0$. Because

$$
\underline{f}_{1}\left(\tau_{2}^{(n)}\right) \leq \tau_{1}^{(n-1)} \text { and } \quad \theta_{2}<\tau_{2}^{(n)}
$$

we can choose some $\tilde{\theta}_{2}$ such that $\theta_{2}<\tilde{\theta}_{2}<\tau_{2}^{(n)}$ and $\underline{f}_{1}\left(\tilde{\theta}_{2}\right)<\tau_{1}^{(n-1)}$. Pick $\delta>0$ small enough so that $\tilde{\theta}_{1} \equiv \delta+\underline{f}_{1}\left(\tilde{\theta}_{2}\right)<\tau_{1}^{(n-1)}$. Then $\tilde{\theta}=\left(\tilde{\theta}_{1}, \tilde{\theta}_{2}\right) \in$ $\Gamma_{2}^{(n-1)}$, where $\Gamma_{2}^{(n-1)}=\Gamma_{2} \cap\left\{\theta_{1}<\tau_{1}^{(n-1)}\right\}$. By part (d) of Lemma 4.1, $\varphi(\tilde{\theta})<\infty$ because $\tilde{\theta} \in \Gamma_{2}^{(n-1)}$ and $\varphi_{2}\left(\tilde{\theta}_{1}\right)<\infty$. Therefore, $\varphi(\tilde{\theta})<\infty$, which implies that $\varphi_{1}\left(\tilde{\theta}_{2}\right)<\infty$ by part (a) of Lemma 4 .1. Because $\theta_{2}<\tilde{\theta}_{2}$, we have $\varphi_{1}\left(\theta_{2}\right)<\infty$. We can prove similarly that $\theta_{1}<\tau_{1}^{(n)}$ implies $\varphi_{2}\left(\theta_{1}\right)<\infty$. This completes the induction argument. Now the conclusion of part (a) follows from (3.8), Lemmas 3.1 and 3.2.

In the following lemma, $\theta>\theta^{(1, r)}$ means that $\theta_{1}>\theta_{1}^{(1, \mathrm{r})}$ and $\theta_{2}>\theta_{2}^{(1, \mathrm{r})}$.

LemmA 5.2. (a) If $\tau_{1}=\theta_{1}^{(1, \mathrm{r})}$, then $\theta>\theta^{(1, \mathrm{r})}$ implies $\varphi(\theta)=\infty$. Similarly, if $\tau_{2}=\theta_{2}^{(2, \mathrm{r})}$, then $\theta>\theta^{(2, \mathrm{r})}$ implies $\varphi(\theta)=\infty$. (b) If $\tau_{1}=$ 


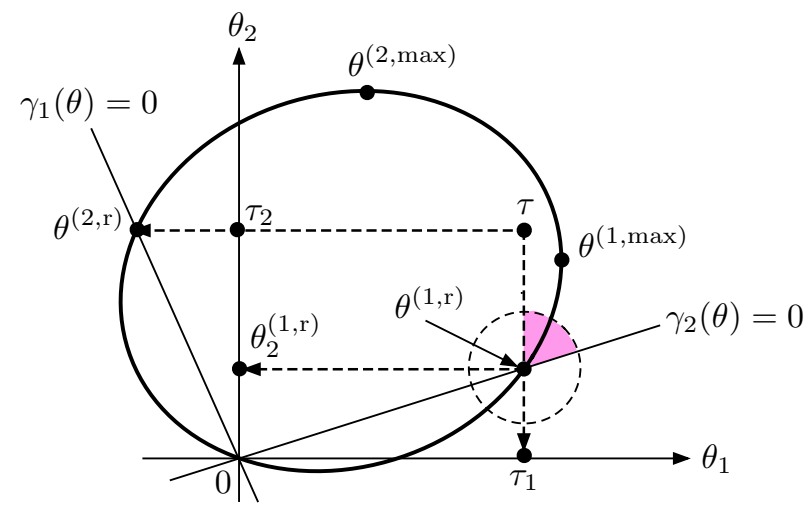

FIG 7. The area for $\theta^{(1, \mathrm{r})}<\eta, \eta_{2}<\tau_{2},\left\|\eta-\theta^{(1, \mathrm{r})}\right\|<\epsilon, \gamma_{1}(\eta)>0, \gamma_{2}(\eta)>0$

$\theta_{1}^{(1, \mathrm{r})}<\theta_{1}^{(1, \max )}$ and $\theta_{2}^{(1, \mathrm{r})}<\tau_{2}$, then $\varphi_{2}\left(\theta_{1}\right)=\infty$ for $\theta_{1}>\tau_{1}$. Similarly, if $\tau_{2}=\theta_{2}^{(2, \mathrm{r})}<\theta_{2}^{(2, \max )}$ and $\theta_{1}^{(2, \mathrm{r})}<\tau_{1}$, then $\varphi_{1}\left(\theta_{2}\right)=\infty$ for $\theta_{2}>\tau_{2}$.

Proof. (a) By the symmetry, it is sufficient to prove $\varphi(\theta)=\infty$ for $\theta>$ $\theta^{(1, \mathrm{r})}$ when $\tau_{1}=\theta_{1}^{(1, \mathrm{r})}$. Because $\gamma\left(\theta^{(1, \mathrm{r})}\right)=0, \gamma_{2}\left(\theta^{(1, \mathrm{r})}\right)=0$, and $\gamma_{1}\left(\theta^{(1, \mathrm{r})}\right)>0$, for each $\epsilon>0$ we can find an $\eta \in \mathbb{R}^{2}$ such that

$$
\left\|\eta-\theta^{(1, \mathrm{r})}\right\|<\epsilon, \quad \gamma(\eta)<0, \quad \gamma_{1}(\eta)>0, \quad \gamma_{2}(\eta)>0,
$$

where, for a $x \in \mathbb{R}^{2},\|x\|$ denotes the Euclidean norm of $x$. Suppose that $\varphi(\eta)<\infty$. Then, $\varphi_{1}\left(\eta_{2}\right)<\infty$ and $\varphi_{2}\left(\eta_{1}\right)<\infty$ by part (b) of Lemma 4.1 for $\eta$. Thus, the key relationship (2.3) holds, but its left side is negative while its right side is positive. This is a contradiction. Thus, we must have $\varphi(\eta)=\infty$, which implies $\varphi(\theta)=\infty$ for any $\theta>\eta$. Since $\epsilon$ can be an arbitrary small positive number, we have proved (a).

(b) Assume $\tau_{1}=\theta_{1}^{(1, \mathrm{r})}<\theta_{1}^{(1, \max )}$ and $\theta_{2}^{(1, \mathrm{r})}<\tau_{2}$. For each $\epsilon>0$, we can find a point $\eta \in \Gamma$ such that $\eta>\theta^{(1, \mathrm{r})}, \eta_{2}<\tau_{2}$ and

$$
\left\|\eta-\theta^{(1, \mathrm{r})}\right\|<\epsilon, \quad \gamma_{1}(\eta)>0, \quad \gamma_{2}(\eta)>0 .
$$

See Figure 7. From Lemma 5.1, $\varphi_{1}\left(\eta_{2}\right)<\infty$. Now we claim $\varphi_{2}\left(\eta_{1}\right)=\infty$. To see this, suppose on the contrary that $\varphi_{2}\left(\eta_{1}\right)<\infty$. Then part (c) of Lemma 4.1 implies $\varphi(\eta)<\infty$, but this contradicts part (a) of this lemma because $\eta>\theta^{(1, \mathrm{r})}$. Thus, we have $\varphi_{2}\left(\eta_{1}\right)=\infty$. Because $\epsilon$ can be an arbitrary positive small number, we concludes $\varphi_{2}\left(\theta_{1}\right)=\infty$ for $\theta_{1}>\theta_{1}^{(1, \mathrm{r})}=\tau_{1}$. The remaining case can be proved analogously by symmetry. 
In the case when $\tau \in \Gamma$ (see Figure 10), Lemma 5.2 gives a complete converse. Therefore, Lemmas 5.1 and 5.2 give a complete proof of Theorem 2.1 when $\tau \in \Gamma$; namely,

$$
\mathcal{D}=\left\{\theta \in \mathbb{R}^{2}: \theta<\tau\right\} .
$$

In general, to establish the complete converse $\mathcal{D} \subset \Gamma_{\max }^{(\tau)}$, in addition to Lemma 5.2, we need to show that $\varphi_{2}\left(\theta_{1}\right)=\infty$ when $\theta_{1}>\tau_{1}$ in the case of $\tau_{1}=\theta_{1}^{(1, \max )}$ or $\tau_{1}=\bar{f}_{1}\left(\tau_{2}\right)$ and that $\varphi_{1}\left(\theta_{2}\right)=\infty$ when $\theta_{2}>\tau_{2}$ in the case of $\tau_{2}=\theta_{2}^{(2, \max )}$ or $\tau_{2}=\bar{f}_{2}\left(\tau_{1}\right)$. We also need to verify that, if $\theta>\tau$ and $\theta \notin \Gamma_{\max }$, then $\varphi(\theta)=\infty$. These cases will be covered in Section 7.1 after the discussion of complex variable functions and their analytic extensions in the next section.

6. Analytic extensions and singular points. Recall that a complex function $g(z)$ is said to be analytic at $z_{0} \in \mathbb{C}$ if there exists a sequence $\left\{a_{n}\right\} \subset \mathbb{C}$ and some $\epsilon>0$ such that

$$
\sum_{n=0}^{\infty} a_{n}\left(z-z_{0}\right)^{n}
$$

is absolutely convergent and is equal to $g(z)$ for each $z \in \mathbb{C}$ with $\left|z-z_{0}\right|<\epsilon$. A point $z \in \mathbb{C}$ is said to be a singular point of a (complex variable) function if the function is not analytic at $z$. A moment generating function for a nonnegative random variable can be considered as a function of complex variable $z \in \mathbb{C}$ that is analytic, at least when the real part $\Re z$ of $z$ is negative. We use complex variable functions to identify the convergence domain $\mathcal{D}$ through their singular points. To this end, the following lemma is useful although it is elementary. The lemma corresponds with Pringsheim's theorem for a generating function (e.g., see Theorem 17.13 in Volume 1 of [23]).

Lemma 6.1. Let $g(\lambda)=\int_{0}^{\infty} e^{\lambda x} d F(x)$ be the moment generating function of a probability distribution $F$ on $\mathbb{R}_{+}$with real variable $\lambda$. Define the convergence parameter of $g$ as

$$
c_{p}(g)=\sup \{\lambda \geq 0 ; g(\lambda)<\infty\} .
$$

Then, the complex variable function $g(z)$ is analytic on $\left\{z \in \mathbb{C} ; \Re z<c_{p}(g)\right\}$ and is singular at $z=c_{p}(g)$.

Remark 6.1. For a complex function $g: \mathbb{C} \rightarrow \mathbb{C}$ that is singular at $z=z_{0} \in \mathbb{C}, g\left(z_{0}\right)$ may be finite at $z_{0}$, but Lemma 6.1 shows that, if $g(z)$ is a moment generating function, then $g(x)=\infty$ for $x \in\left(\Re z_{0}, \infty\right)$.

The following corollary is immediate from this lemma. 
Corollary 6.1. Under the same notation of Lemma 6.1, we have the following two facts.

(a) If $g$ is analytically extendable from $\{z \in \mathbb{C} ; \Re z<0\}$ to an open set including a real segment $\left[0, \lambda_{1}\right]$ for some $\lambda_{1}>0$, then $g\left(\lambda_{1}\right)<\infty$.

(b) If $g(z)$ is singular at $z=\lambda_{0}$ for some real number $\lambda_{0}$, then $\lambda_{0} \geq$ $c_{p}(g)$.

From Lemmas 5.1 and 6.1 and (c) of Lemma 4.1, we have the following lemma.

Lemma 6.2. The complex function $\varphi_{1}(z)$ is analytic for $\Re z<\tau_{1}$, the complex function $\varphi_{2}(z)$ is analytic for $\Re z<\tau_{2}$.

The remaining of this section is to prove that $\varphi_{k}(z)$ is singular at $z=\tau_{k}$ and to determine the nature of singularity at $z=\tau_{k}, k=1,2$. For that, we are going to relate $\varphi_{1}(z)$ and $\varphi_{2}(z)$ through (2.3). This motivates us to study the roots of $\gamma(\theta)=0$. For that, it is convenient to have function representations for different segments of the ellipse $\partial \Gamma$, which is defined by the equation $\gamma\left(\theta_{1}, \theta_{2}\right)=0$ for $\left(\theta_{1}, \theta_{2}\right) \in \mathbb{R}^{2}$. Recall that $\underline{f}_{2}\left(\theta_{1}\right)$ represents the lower half of the ellipse $\partial \Gamma$ and $f_{1}\left(\theta_{2}\right)$ represents the left half of the ellipse $\partial \Gamma$. As it will be clear from (6.9) and (6.8) below that $\underline{f}_{1}$ and $\underline{f}_{2}$ play key roles for finding the singularities of $\varphi_{1}(z)$ and $\varphi_{2}(z)$. Recall again that $\theta^{(1, \max )}$ is the right-most point on $\partial \Gamma$ and $\theta^{(2, \max )}$ is the highest point on $\partial \Gamma$. Define $\theta^{(1, \mathrm{~min})}$ to be the left-most point on $\partial \Gamma$ and $\theta^{(2, \mathrm{~min})}$ to be lowest point on $\partial \Gamma$. Then, $\underline{f}_{2}:\left[\theta_{1}^{(1, \min )}, \theta_{1}^{(1, \max )}\right] \rightarrow\left[\theta_{2}^{(2, \min )}, \theta_{2}^{(2, \max )}\right]$ is well defined, and is given by formula

$$
\begin{aligned}
\underline{f}_{2}\left(\theta_{1}\right)= & \frac{1}{\Sigma_{22}}\left(-\left(\mu_{2}+\Sigma_{12} \theta_{1}\right)\right. \\
& \left.-\sqrt{\left(\mu_{2}+\Sigma_{12} \theta_{1}\right)^{2}-\Sigma_{22}\left(\Sigma_{11} \theta_{1}^{2}+2 \mu_{1} \theta_{1}\right)}\right)
\end{aligned}
$$

for $\theta_{1} \in\left[\theta_{1}^{(1, \min )}, \theta_{1}^{(1, \max )}\right]$. A formula for $\underline{f}_{1}\left(\theta_{2}\right)$ can be written down similarly.

The following lemma says that $f_{2}(z)$ has an analytic extension. Recall that, for a multi-valued function $\overline{f(z)}$ on $\mathbb{C}$, a point $z_{0} \in \mathbb{C}$ is said to be a branch point of $f$ if there exists a neighborhood such that an arbitrary closed continuous curve around $z_{0}$ in the neighborhood carries each branch of $f$ to another branch of $f$ (see Section 11 in Volume I of [23] for its precise definition). For example, $f(z)=\left(z-z_{0}\right)^{1 / n}$ with integer $n \geq 2$ is an $n$-valued function with branch point $z_{0}$. The integer $n$ is referred to as the multiplicity of the branch point. Obviously, each branch of this function is analytic on 
$\mathcal{G}_{\delta}\left(z_{0}\right)$, where, for $\delta \in[0, \pi / 2)$,

$$
\mathcal{G}_{\delta}\left(z_{0}\right)=\left\{z \in \mathbb{C}: z \neq z_{0}, \quad\left|\arg \left(z-z_{0}\right)\right|>\delta\right\},
$$

and $\arg z \in(-\pi, \pi)$ is the principal part of the argument of a complex number $z$.

Since $\underline{f}_{2}(z)$ is multivalued for complex number $z \in \mathbb{C}$, we take its branch such that $\underline{f}_{2}(z)=\underline{f}_{2}(\Re z)$ for $z \in\left(\theta_{1}^{(1, \min )}, \theta_{1}^{(1, \max )}\right)$. We use the same notation $\underline{f}_{2}(z)$ for this branch throughout the paper.

LEMma 6.3. $\underline{f}_{2}$ is analytic on the whole complex plain except for the two half real lines $\left(-\infty, \theta_{1}^{(1, \min )}\right)$ and $\left(\theta_{1}^{(1, \max )}, \infty\right)$, and has branch points at $z=\theta_{1}^{(1, \min )}$ and $\theta_{1}^{(1, \max )}$, each with multiplicity two. Furthermore,

$$
\begin{array}{ll}
\Re \underline{f}_{2}(z) \leq \underline{f}_{2}(\Re z), \quad \Re z \in\left(\theta_{1}^{(1, \min )}, \theta_{1}^{(1, \max )}\right), \\
\Re \underline{f}_{2}(z) \leq \theta_{2}^{(1, \max )}, \quad z \in \mathcal{G}_{\delta}\left(\theta_{1}^{(1, \max )}\right) \cap\left\{u \in \mathbb{C} ; \Re u>\theta_{1}^{(1, \min )}\right\},
\end{array}
$$

for some $\delta \in\left[0, \frac{\pi}{2}\right)$.

Proof. Because $\theta_{1}^{(1, \min )}$ and $\theta_{1}^{(1, \max )}$ are two roots of the quadratic equation

$$
\left(\mu_{2}+\Sigma_{12} \eta_{1}\right)^{2}-\Sigma_{22}\left(\Sigma_{11} \eta_{1}^{2}+2 \mu_{1} \eta_{1}\right)=0,
$$

the quadratic function must be equal to

$$
\operatorname{det}(\Sigma)\left(\eta_{1}-\theta_{1}^{(1, \min )}\right)\left(\theta^{(1, \max )}-\eta_{1}\right)
$$

where $\operatorname{det}(\Sigma)=\Sigma_{11} \Sigma_{22}-\Sigma_{12}^{2}$. Thus the complex variable version of $\underline{f}_{2}$ in (6.2) must have the following representation:

$$
\underline{f}_{2}(z)=-\frac{\mu_{2}+\Sigma_{12} z}{\Sigma_{22}}-\frac{\sqrt{\operatorname{det}(\Sigma)}}{\Sigma_{22}} \sqrt{\left(z-\theta_{1}^{(1, \min )}\right)\left(\theta_{1}^{(1, \max )}-z\right)} .
$$

Let

$$
g(z)=\sqrt{\left(z-\theta_{1}^{(1, \min )}\right)\left(\theta_{1}^{(1, \max )}-z\right)} .
$$

Then, from our choice of the branch of $\underline{f}_{2}, g(z)$ can be written as

$$
\sqrt{\left|\left(\theta_{1}^{(1, \max )}-z\right)\left(z-\theta_{1}^{(1, \min )}\right)\right|} \exp \left(i \frac{\omega_{-}(z)+\omega_{+}(z)}{2}\right),
$$

where $\omega_{+}(z)=\arg \left(z-\theta_{1}^{(1, \min )}\right)$ and $\omega_{-}(z)=\arg \left(\theta_{1}^{(1, \max )}-z\right)$ (see Figure 8$)$. 


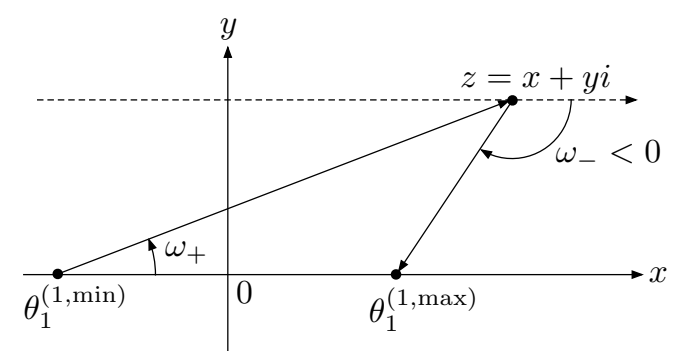

FIG 8. $\omega_{+}(z)=\arg \left(z-\theta_{1}^{(1, \min )}\right)>0$ and $\omega_{-}(z)=\arg \left(\theta_{1}^{(1, \max )}-z\right)<0$

Thus, $\underline{f}_{2}$ is analytic except for the two half lines, and has two branch points at $\theta_{1}^{(1, \min )}$ and $\theta_{1}^{(1, \max )}$ as claimed. Note that $\omega_{-}(z), \omega_{+}(z) \in(-\pi, \pi)$ and their signs are different if $z$ is not on the real line. Hence, $-\frac{\pi}{2}<$ $\frac{\omega_{-}(z)+\omega_{+}(z)}{2}<\frac{\pi}{2}$. This and the fact that

$$
\Re g(z)=\sqrt{\left|\left(\theta_{1}^{(1, \max )}-z\right)\left(z-\theta_{1}^{(1, \min )}\right)\right|} \cos \left(\frac{\omega_{-}(z)+\omega_{+}(z)}{2}\right)
$$

imply $\Re g(z)>0$ for $z$ not on the two half lines.

We now prove (6.2). For this, it suffices to prove that for any $z \in \mathbb{C}$ with $\Re z \in\left(\theta_{1}^{(1, \min )}, \theta_{1}^{(1, \max )}\right), \Re g(z) \geq g(\Re z)$.

For $z \in \mathbb{C}$ satisfying $\Re z \in\left(\theta_{1}^{(1, \min )}, \theta_{1}^{(1, \max )}\right), \omega_{-}(z), \omega_{+}(z) \in\left(-\frac{\pi}{2}, \frac{\pi}{2}\right)$, so we have $\cos \omega_{+}(z) \geq 0$ and $\cos \omega_{-}(z) \geq 0$. Hence,

$$
\begin{aligned}
& \left(\theta_{1}^{(1, \max )}-\Re z\right)\left(\Re z-\theta_{1}^{(1, \text { min })}\right)= \\
& \quad\left|\left(\theta_{1}^{(1, \max )}-z\right)\left(z-\theta_{1}^{(1, \min )}\right)\right| \cos \omega_{-}(z) \cos \omega_{+}(z) \geq 0 .
\end{aligned}
$$

Thus, $\Re g(z) \geq g(\Re z)$ is obtained if we show that

$$
\left(\cos \frac{\omega_{+}(z)+\omega_{-}(z)}{2}\right)^{2} \geq \cos \omega_{-}(z) \cos \omega_{+}(z) .
$$

This indeed holds because

$$
\begin{aligned}
\left(\cos \frac{\omega_{+}(z)+\omega_{-}(z)}{2}\right)^{2}=\frac{1}{2}\left(1+\cos \left(\omega_{-}(z)+\omega_{+}(z)\right)\right) \\
=\frac{1}{2}\left(1+\cos \omega_{-}(z) \cos \omega_{+}(z)-\sin \omega_{-}(z) \sin \omega_{+}(z)\right) \\
=\cos \omega_{-}(z) \cos \omega_{+}(z) \\
\quad+\frac{1}{2}\left(1-\left(\cos \omega_{-}(z) \cos \omega_{+}(z)+\sin \omega_{-}(z) \sin \omega_{+}(z)\right)\right) \\
=\quad \cos \omega_{-}(z) \cos \omega_{+}(z) \\
\quad+\frac{1}{2}\left(1-\cos \left(\omega_{-}(z)-\omega_{+}(z)\right)\right) \geq \cos \omega_{-}(z) \cos \omega_{+}(z) .
\end{aligned}
$$


It remains to prove (6.3). From (6.4) and (6.5), we have

$$
\begin{aligned}
\underline{f}_{2}(z & \left.+\theta_{1}^{(1, \max )}\right)-\theta_{2}^{(1, \max )}=-\frac{\Sigma_{12} z}{\Sigma_{22}} \\
& -\frac{\sqrt{\operatorname{det}(\Sigma)}}{\Sigma_{22}} \sqrt{|z|\left|z+\bar{\theta}_{1}\right|} \exp \left(i \frac{\arg (-z)+\arg \left(z+\bar{\theta}_{1}\right)}{2}\right)
\end{aligned}
$$

where $\bar{\theta}_{1}=\theta_{1}^{(1, \max )}-\theta_{1}^{(1, \min )}$. Let $z \in \mathbb{C}$ satisfy $\Re z \geq 0$ and $z$ is not real. Then, $z$ can be expressed as $z=r e^{i \omega_{0}}$ with $r>0$ and $\omega_{0} \in\left[-\frac{\pi}{2}, \frac{\pi}{2}\right] \backslash\{0\}$. Similarly, let $z+\bar{\theta}_{1}=s e^{i \omega_{1}}$ with $\omega_{1} \in\left[-\frac{\pi}{2}, \frac{\pi}{2}\right] \backslash\{0\}$. Clearly, $r<s$. Since $z$ and $z+\bar{\theta}_{1}$ have the same imaginary component, $\omega_{0}$ and $\omega_{1}$ have the same sign. Hence, it is sufficient to consider for $\omega_{0}, \omega_{1}>0$ to prove the right side of (6.6) to be less than zero. In this case, it is easy to see that $\arg (-z)=\omega_{0}-\pi$ and $\omega_{1}<\omega_{0}$. Using these notation, it follows from (6.6) that

$$
\begin{aligned}
\Re \underline{f}_{2}(z & \left.+\theta_{1}^{(1, \max )}\right)-\theta_{2}^{(1, \max )} \\
& =-\frac{\Sigma_{12}}{\Sigma_{22}} r \cos \omega_{0}-\frac{\sqrt{\operatorname{det}(\Sigma)}}{\Sigma_{22}} \sqrt{r s} \cos \left(\frac{\omega_{0}-\pi+\omega_{1}}{2}\right) \\
& =-\frac{\Sigma_{12}}{\Sigma_{22}} r \cos \omega_{0}-\frac{\sqrt{\operatorname{det}(\Sigma)}}{\Sigma_{22}} \sqrt{r s} \sin \left(\frac{\omega_{0}+\omega_{1}}{2}\right) \\
& \leq-\frac{r}{\Sigma_{22}}\left(\Sigma_{12} \cos \omega_{0}+\sqrt{\operatorname{det}(\Sigma)} \sqrt{\frac{s}{r}} \sin \left(\frac{\omega_{0}}{2}\right)\right) \\
& \leq-\frac{r}{\Sigma_{22}}\left(\Sigma_{12} \cos \omega_{0}+\sqrt{\operatorname{det}(\Sigma)} \sin \left(\frac{\omega_{0}}{2}\right)\right)
\end{aligned}
$$

If $\Sigma_{12} \geq 0$, then the last term of this inequality is negative, and therefore (6.6) is negative. Hence, (6.3) holds for $\delta=0$. Otherwise, we choose $\delta \in$ $\left(0, \frac{\pi}{2}\right)$ such that

$$
\frac{\sin \left(\frac{\delta}{2}\right)}{\cos \delta}>\frac{\sqrt{\operatorname{det}(\Sigma)}}{\left|\Sigma_{12}\right|},
$$

which is possible because its left side goes to infinity as $\delta \uparrow \frac{\pi}{2}$. Then, for $\omega_{0}>\delta$, we have, by the monotonicity of sine and cosine functions on the interval $\left[0, \frac{\pi}{2}\right]$,

$$
\frac{\sin \left(\frac{\omega_{0}}{2}\right)}{\cos \omega_{0}}>\frac{\sin \left(\frac{\delta}{2}\right)}{\cos \delta}>\frac{\sqrt{\operatorname{det}(\Sigma)}}{\left|\Sigma_{12}\right|}
$$

This implies that the right side of (6.7) is negative. Thus, combining this result with (6.2), we obtain (6.3). This completes the proof. 
From Lemma 6.2, $\varphi_{1}(z)$ is analytic in $\Re z<\tau_{2}$ and $\varphi_{2}(z)$ is analytic in $\Re z<\tau_{1}$. Clearly $f_{1}(z)$ has an analytic extension that is similar to the one in Lemma 6.3 for $f_{2}(z)$. Using $f_{1}(z)$ and $f_{2}(z)$, we now consider analytic extensions for $\varphi_{1}(z)$ and $\varphi_{2}(z)$. Note that $\underline{f}_{1}\left(\tau_{2}\right) \leq \tau_{1}$ and $\underline{f}_{2}\left(\tau_{1}\right) \leq \tau_{2}$. In the case when the inequalities are strict, the following lemma shows that $\varphi_{1}(z)$ and $\varphi_{2}(z)$ can be extended analytically to strictly larger domains.

Lemma 6.4. (a) The complex variable function $\gamma_{2}\left(z, \underline{f}_{2}(z)\right)$ is analytic on

$$
\mathbb{C} \backslash\left(-\infty, \theta_{1}^{(1, \min )}\right] \cup\left[\theta_{1}^{(1, \max )}, \infty\right) .
$$

(b) $\varphi_{2}$ is analytically extendable for $z \in \mathbb{C} \backslash\left(-\infty, \theta_{1}^{(1, \min )}\right] \cup\left[\theta_{1}^{(1, \max )}, \infty\right)$ satisfying $\gamma_{2}\left(z, \underline{f}_{2}(z)\right) \neq 0$ and $\Re \underline{f}_{2}(z)<\tau_{2}$, and

$$
\varphi_{2}(z)=-\frac{\gamma_{1}\left(z, \underline{f}_{2}(z)\right) \varphi_{1}\left(\underline{f}_{2}(z)\right)}{\gamma_{2}\left(z, \underline{f}_{2}(z)\right)} .
$$

Similarly, $\varphi_{1}$ is analytically extendable for $z \in \mathbb{C} \backslash\left(-\infty, \theta_{2}^{(2, \min )}\right] \cup\left[\theta_{2}^{(2, \max )}, \infty\right)$ satisfying $\Re \underline{f}_{1}(z)<\tau_{1}$ and $\gamma_{1}\left(\underline{f}_{1}(z), z\right) \neq 0$, and has the expression:

$$
\varphi_{1}(z)=-\frac{\gamma_{2}\left(\underline{f}_{1}(z), z\right) \varphi_{2}\left(\underline{f}_{1}(z)\right)}{\gamma_{1}\left(\underline{f}_{1}(z), z\right)} .
$$

Proof. Whenever $f_{2}(z)$ is analytic at a point $z$, so is $\gamma_{2}\left(z, f_{2}(z)\right)$. Thus, we have (a). To prove (b), we first prove (6.8). We claim that for $0<\Re z<\tau_{1}$,

$$
\gamma_{2}\left(z, \underline{f}_{2}(z)\right) \varphi_{2}(z)=-\gamma_{1}\left(z, \underline{f}_{2}(z)\right) \varphi_{1}\left(\underline{f}_{2}(z)\right) .
$$

To see this, observe that both sides of (6.10) is analytic in $0<\Re z<\tau_{1}$ because $\underline{f}_{2}$ is analytic in $\mathbb{C} \backslash\left(\left(-\infty, \theta_{1}^{(1, \min )}\right] \cup\left[\theta_{1}^{(1, \max )}, \infty\right)\right), \varphi_{1}(z)$ is analytic in $\Re z<\tau_{2}, \varphi_{2}(z)$ is analytic in $\Re z<\tau_{1}, \underline{f}_{2}\left(\theta_{1}\right) \leq \tau_{2}$ for $\theta_{1} \in\left[0, \tau_{1}\right]$, and (6.2) holds. For each $\theta_{1} \in\left(0, \tau_{1}\right)$, we have $\underline{f}_{2}^{2}\left(\theta_{1}\right)<\tau_{2}$. Choose $\epsilon>0$ small enough such that $\underline{f}_{2}\left(\theta_{1}\right)+\epsilon<\tau_{2}$ and $\left(\theta_{1}, \underline{f}_{2}\left(\theta_{1}\right)+\epsilon\right) \in \Gamma$. By part (c) of Lemma 4.1, $\varphi(\theta)<\infty$ for $\theta=\left(\theta_{1}, f_{2}\left(\theta_{1}\right)+\bar{\epsilon}\right)$. It follows that $\varphi(\theta)<\infty$ for $\theta=\left(\theta_{1}, \underline{f}_{2}\left(\theta_{1}\right)\right)$. Since $\gamma(\theta)=0$ for $\bar{\theta}=\left(\theta_{1}, \underline{f}_{2}\left(\theta_{1}\right)\right)$, it follows from (2.3) that (6.10) holds for $\theta_{1} \in\left(0, \tau_{1}\right)$. By unique analytic extension, (6.10) holds for $0<\Re z<\tau_{1}$, proving the claim. Thus, (6.8) holds for $0<\Re z<\tau_{1}$ satisfying $\gamma_{2}\left(z, \underline{f}_{2}(z)\right) \neq 0$. Because the left side of (6.10) is analytic in $\Re z<\tau_{1}$ and the right side of $(6.10)$ is analytic for $z \in \mathbb{C} \backslash\left(-\infty, \theta_{2}^{(2, \min )}\right] \cup\left[\theta_{2}^{(2, \max )}, \infty\right)$ satisfying $\Re \underline{f}_{2}(z)<\tau_{2}$ and $\gamma_{2}\left(z, \underline{f}_{2}(z)\right) \neq 0$, we have (6.8) for the specified region. The proof of (6.9) is analogous. 


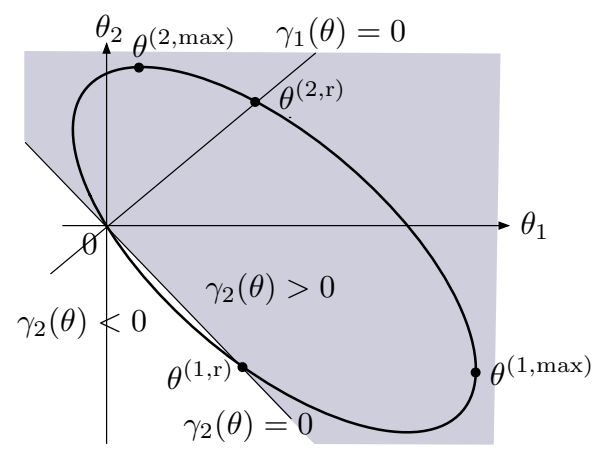

FIG 9. An example in which point $\theta^{(1, \mathrm{r})}$ is on the lower half of the ellipse, but $\theta_{2}^{(1, \mathrm{r})}>$ $\theta_{2}^{(1, \max )}$

Representations (6.8) and (6.9) play a key role in determining the singularities of $\varphi_{1}(z)$ and $\varphi_{2}(z)$. The following lemma tells when the denominators in (6.8) and (6.9) are zero.

LEMMA 6.5. (a) $z=0$ is a root of $\gamma_{2}\left(z, \underline{f}_{2}(z)\right)=0$. It has another root, which is equal to $z=\theta_{1}^{(1, \mathrm{r})}$, if and only if $\gamma_{2}\left(\theta^{(1, \max )}\right) \geq 0$. (b) Assume that $\gamma_{2}\left(\theta^{(1, \max )}\right)>0$. Let

$$
\bar{\gamma}_{2}(z)= \begin{cases}\gamma_{2}\left(z, \underline{f}_{2}(z)\right) /\left(\theta_{1}^{(1, \mathrm{r})}-z\right), & \text { if } z \neq \theta_{1}^{(1, \mathrm{r})} \\ \gamma_{2}\left(1, \underline{f}_{2}^{\prime}\left(\theta_{1}^{(1, \mathrm{r})}\right)\right), & \text { if } z=\theta_{1}^{(1, \mathrm{r})}\end{cases}
$$

Then $\bar{\gamma}_{2}$ is analytic at $z=\theta_{1}^{(1, \mathrm{r})}$. (c) $\bar{\gamma}_{2}\left(\theta_{1}^{(1, \mathrm{r})}\right) \neq 0$. Thus, $1 / \gamma_{2}\left(z, \underline{f}_{2}(z)\right)$ has a simple pole at $z=\theta_{1}^{(1, \mathrm{r})}$ when $\gamma_{2}\left(\theta^{(1, \max )}\right)>0$. Analogous results also hold for $\gamma_{1}\left(\underline{f}_{1}(z), z\right)$.

Proof. To prove (a), note that each root of $\gamma_{2}\left(z, \underline{f}_{2}(z)\right)=0$ must be a root of a quadratic equation with real coefficients. Thus, there are at most two roots for $\gamma_{2}\left(z, \underline{f}_{2}(z)\right)=0$. Since $z=0$ is a solution, the other solution must also be real. Note that $\gamma_{2}\left(\theta^{(1, \max )}\right) \geq 0$ if and only if the point $\theta^{(1, \mathrm{r})}$ is on the lower half of the ellipse; see Figure 9 for an illustration. Thus, when $\gamma_{2}\left(\theta^{(1, \max )}\right) \geq 0, \theta_{1}^{(1, \mathrm{r})}$ is the other root. When $\gamma_{2}\left(\theta^{(1, \max )}\right)<0$, the line $\gamma_{2}(\theta)=0$ does not intersect with the lower half of the ellipse $\partial \Gamma$ except at the origin. Thus, $\gamma_{2}\left(z, f_{2}(z)\right)=0$ has no other root. Part (a) yields part (b) because of part (a) of Lemma 6.4. Part (c) holds because the tangent of the ellipse at $\theta^{(1, r)}$ cannot be orthogonal to line $\gamma_{2}(\theta)=0$ when $\theta^{(1, \mathrm{r})} \neq \theta^{(1, \max )}$. 
The following lemma classifies the singularity of $\varphi_{2}(z)$ at $z=\tau_{1}$ when $\tau_{1}<\theta_{1}^{(1, \max )}$. For an $x>0$ and $z_{0} \in \mathbb{C}$, we denote the ball in $\mathbb{C}$ that has center $z_{0}$ and radius $x$ by $B_{x}\left(z_{0}\right)$, that is, $B_{x}\left(z_{0}\right)=\left\{z \in \mathbb{C}:\left|z-z_{0}\right|<x\right\}$.

Lemma 6.6. Assume that $\tau_{1}<\theta_{1}^{(1, \max )}$. (a) In Categories I and III, $\tau_{1}=\theta_{1}^{(1, \mathrm{r})}$ and $\varphi_{2}(z)$ has a simple pole at $z=\tau_{1}$. (b) In Category II, $\varphi_{2}(z)$ has a simple pole at $z=\tau_{1}$ if $\tau_{1} \neq \theta_{1}^{(1, \mathrm{r})}$. It has a double pole at $z=\tau_{1}$ if $\tau_{1}=\theta_{1}^{(1, \mathrm{r})}$. (c) There exists an $\epsilon>0$ such that $\varphi_{2}(z)$ is analytic for $\Re z \leq \tau_{1}+\epsilon$ except for $z=\tau_{1}$ and for each $a>0$

$$
\sup _{\substack{z \notin B a\left(\tau_{1}\right) \\ \Re z \leq \tau_{1}+\epsilon}}\left|\varphi_{2}(z)\right|<\infty .
$$

Proof. Assume $\tau_{1}<\theta_{1}^{(1, \max )}$. We first prove (a) and (c) for Categories I and III. In this case, $\tau_{1}=\theta_{1}^{(1, \mathrm{r})}$ and $\gamma_{2}\left(\theta^{(1, \max )}\right) \geq 0$. By Lemma 6.4, $\varphi_{2}(z)$ has representation (6.8). The numerator of (6.8) is analytic on on $\mathbb{C} \backslash\left(-\infty, \theta_{1}^{(1, \min )}\right]$ as long as $\Re \underline{f}_{2}(z)<\tau_{2}$. The latter condition is satisfied if $\theta_{1}^{(1, \min )}<\Re z<\theta_{1}^{(1, \max )}$ and

$$
\underline{f}_{2}(\Re z)<\tau_{2},
$$

because of (6.2).

When $\tau_{2} \geq \theta_{2}^{(1, \max )}$, condition (6.12) is satisfied if $0 \leq \Re z<\theta_{1}^{(1, \max )}$, and we choose $\epsilon>0$ to satisfy

$$
\tau_{1}+2 \epsilon=\theta_{1}^{(1, \max )}
$$

When $\tau_{2}<\theta_{2}^{(1, \max )}$, condition (6.12) is satisfied if $0 \leq \Re z<\bar{f}_{1}\left(\tau_{2}\right)$ (see, Figure 10 for a case in Category I). Now we argue that

$$
\tau_{1}<\bar{f}_{1}\left(\tau_{2}\right) \text { when } \tau_{2}<\theta_{2}^{(1, \max )} \text { and } \tau_{1}<\theta_{1}^{(1, \max )} .
$$

To prove (6.13) we note that $\bar{f}_{1}(x)$ is strictly increasing in $\left[\theta_{2}^{2, \min }, \theta_{2}^{(1, \max )}\right]$. Thus, when $\tau_{1}<\theta_{1}^{(2, \text { min })}$, we have $\bar{f}_{1}\left(\tau_{2}\right) \geq \bar{f}_{1}\left(\theta_{2}^{(2, \min )}\right)=\theta_{1}^{(2, \min )}>\tau_{1}$. It remains to prove $(6.13)$ when $\tau_{1} \geq \theta_{1}^{(2, \min )}$ and $\tau_{2}<\theta_{2}^{(1, \max )}$. Assume that $\theta_{1}^{(2, \min )} \leq \tau_{1}<\theta_{1}^{(1, \max )}$ and $\tau_{2}<\theta_{2}^{(1, \max )}$. In this case, $\bar{f}_{1}\left(\underline{f}_{2}\left(\tau_{1}\right)\right)=\tau_{1}$. Because $\theta_{2}^{2 \text {,min }} \leq \underline{f}_{2}\left(\tau_{1}\right) \leq \tau_{2} \leq \theta_{2}^{(1, \max )}$, by the increasing property of function $\bar{f}_{1}$, we have that condition $\tau_{1}<\bar{f}_{1}\left(\tau_{2}\right)$ is equivalent to $\underline{f}_{2}\left(\tau_{1}\right)<\tau_{2}$. The latter condition is satisfied for Category I when $\tau_{1}<\theta_{1}^{(1, \max )}$. Condition 


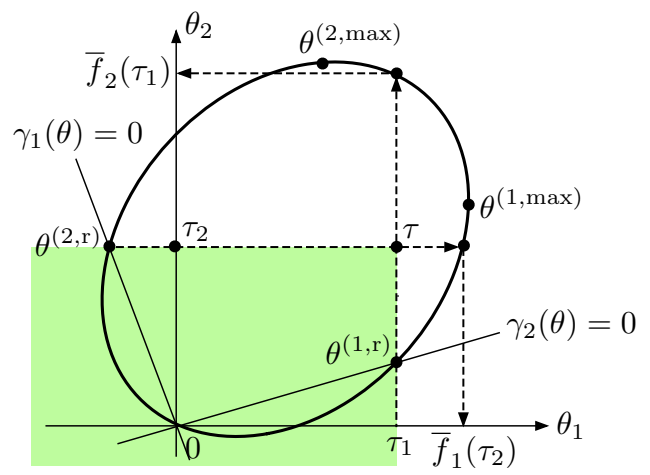

FIG 10. A case in Category I: $\varphi_{2}(z)$ has a simple pole at $\tau_{1}$ and has the second singularity at $\bar{f}_{1}\left(\tau_{2}\right) \in\left(\tau_{1}, \theta_{1}^{(1, \max )}\right)$

$\underline{f}_{2}\left(\tau_{1}\right)<\tau_{2}$ is also satisfied for Category III, because $\tau_{2}=\bar{f}_{2}\left(\tau_{1}\right)>\underline{f}_{2}\left(\tau_{1}\right)$ when $\theta_{1}^{(1, \min )}<\tau_{1}<\theta_{1}^{(1, \max )}$. Thus, we have proved (6.13). Therefore, when $\tau_{2}<\theta_{2}^{(1, \max )}$, we can choose $\epsilon>0$ to satisfy

$$
\tau_{1}+2 \epsilon=\bar{f}_{1}\left(\tau_{2}\right)
$$

In either case, with our choices of $\epsilon>0,(6.2)$ implies that the numerator of (6.8) is analytic on $0 \leq \Re z<\tau_{1}+2 \epsilon$. Since $\gamma_{2}\left(\theta^{(1, \max )}\right)>0$ in either Category I or III, by Lemma $6.5, \gamma_{2}\left(z, \underline{f}_{2}(z)\right)=\bar{\gamma}_{2}(z)\left(\tau_{1}-z\right)$, where $\bar{\gamma}_{2}(z)$ is analytic in $0<\Re z<\tau_{1}+2 \epsilon$ and $\bar{\gamma}_{2}(\bar{z}) \neq 0$ in the region. It follows from (6.8) that $\varphi_{2}(z)$ is analytic on $\Re z<\tau_{1}+2 \epsilon$ except at $z=\tau_{1}$ and it has a simple pole at $z=\tau_{1}$. Because $\underline{f}_{2}\left(\tau_{1}+\epsilon\right)<\tau_{2}$, we have

$$
\varphi_{1}\left(\underline{f}_{2}\left(\tau_{1}+\epsilon\right)\right)<\infty
$$

Also, by Lemma 6.5, for any $a>0$,

$$
\sup _{\substack{z \notin B a\left(\tau_{1}\right) \\ 0 \leq \Re z \leq \tau_{1}+\epsilon}}\left|\frac{\gamma_{1}\left(z, \underline{f}_{2}(z)\right)}{\gamma_{2}\left(z, \underline{f}_{2}(z)\right)}\right|<\infty .
$$

The bound (6.11) follows from (6.8), (6.14), and (6.15).

Now we prove (b) and (c) for Category II. In this case,

$$
\tau_{2}=\theta_{2}^{(2, \mathrm{r})} \leq \min \left(\theta_{2}^{(1, \mathrm{r})}, \theta_{2}^{(1, \max )}\right) \quad \text { and } \quad \tau_{1}=\bar{f}_{1}\left(\tau_{2}\right) .
$$

Because $0 \leq \tau_{1}<\theta_{1}^{(1, \max )}$ by assumption, $\underline{f}_{2}(z)$ is analytic at $z=\tau_{1}$ and $\underline{f}_{2}\left(\tau_{1}\right)=\tau_{2} \leq \theta_{2}^{(1, \max )}$. One can check that $\underline{f}_{2}^{\prime}\left(\tau_{1}\right) \neq 0$ because of $\tau_{1} \neq$ 


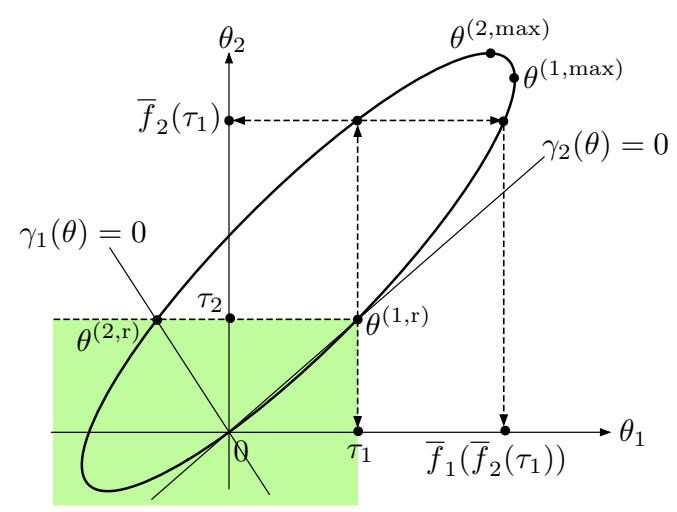

FIG 11. A case in Category II: $\varphi_{2}(z)$ has a pole, either simple or double, at $\tau_{1}$ and has the second singularity at $\bar{f}_{1}\left(\bar{f}_{2}\left(\tau_{1}\right)\right) \in\left(\tau_{1}, \theta_{1}^{(1, \max )}\right)$

$\theta_{1}^{(2, \mathrm{~min})}$. The latter follows from the fact that $\tau_{1}=\theta_{1}^{(2, \mathrm{~min})}$ implies $\tau_{2}=$ $\underline{f}_{2}\left(\tau_{1}\right)=\theta_{2}^{(2, \min )}<0$, which is impossible. By parts (a) and (c) of Lemma 6.7, $\bar{\varphi}_{1}(z)$ has a simple pole at $z=\tau_{2}$. Thus, $\varphi_{1}\left(\underline{f}_{2}(z)\right)$ has a simple pole at $z=\tau_{1}$. By $(6.8)$ and Lemma $6.5, \varphi_{2}(z)$ has a simple pole at $z=\tau_{1}$ if $\tau_{1} \neq \theta_{1}^{(1 . r)}$ and a double pole if $\tau_{1}=\theta_{1}^{(1, \mathrm{r})}$.

It remains to prove that (6.11) holds for each $a>0$. Since $\underline{f}_{2}\left(\tau_{1}\right)=\tau_{2}$ and $\tau_{1}<\theta_{1}^{(1, \max )}$, we can choose an $\epsilon_{1}>0$ small enough such that $\underline{f}_{2}\left(\tau_{1}+\epsilon_{1}\right)<$ $\tau_{2}+\epsilon$, where $\epsilon$ is the constant in (6.7) in Lemma 6.7 below, where parts (a) and (c) of Lemma 6.7 can be proved in exactly the same way for proving parts (a) and (c) of this lemma; the proof for parts (a) and (c) of this lemma has been completed earlier. Because $\sup _{\Re z \leq 0}\left|\varphi_{2}(z)\right|<\infty$, it suffices to prove

$$
\sup _{\substack{z \notin B_{a}\left(\tau_{1}\right) \\ 0 \leq \Re z \leq \tau_{1}+\epsilon_{1}}}\left|\varphi_{2}(z)\right|<\infty
$$

for each $a>0$. Because

$$
\lim _{\theta_{1}^{(1, \min )} \leq \Re \mid \rightarrow \infty}\left|\underline{f}_{2}(z)\right|=\infty
$$

and $\underline{f}_{2}(z)-\tau_{2} \neq 0$ for $z \notin B_{a}\left(\tau_{1}\right)$ with $\theta_{1}^{(1, \min )} \leq \Re z \leq \theta_{1}^{(1, \max )}$, there exists an $a_{1}>0$ such that

$$
\left|\underline{f}_{2}(z)-\tau_{2}\right| \geq a_{1}
$$


for $z \notin B_{a}\left(\tau_{1}\right)$ with $\theta_{1}^{(1, \min )} \leq \Re z \leq \theta_{1}^{(1, \max )}$. It follows from (6.16) and (6.2) that

$$
\sup _{\substack{z \notin B_{a}\left(\tau_{1}\right) \\ 0 \leq \Re z \leq \tau_{1}+\epsilon_{1}}}\left|\varphi_{1}\left(\underline{f}_{2}(z)\right)\right| \leq \sup _{\substack{z \notin B_{1}\left(\tau_{2}\right) \\ 0 \leq \Re z \leq \tau_{2}+\epsilon}}\left|\varphi_{1}(z)\right|<\infty,
$$

where the last inequality follows from (6.7). When $\tau_{1}=\theta_{1}^{(1, \mathrm{r})}$, bound (6.11) with $\epsilon=\epsilon_{1}$ follows from (6.8), (6.15), and (6.17). When $\tau_{1}<\theta_{1}^{(1, \mathrm{r})}$, choose $\epsilon_{1}>0$ small enough so that $\tau_{1}+\epsilon_{1}<\theta_{1}^{(1, \mathrm{r})}$ in addition to $\underline{f}_{2}\left(\tau_{1}+\epsilon_{1}\right) \leq \tau_{2}+\epsilon$. Then, by Lemma 6.5,

$$
\sup _{0 \leq \Re z \leq \tau_{1}+\epsilon_{1}}\left|\frac{\gamma_{1}\left(z, \underline{f}_{2}(z)\right)}{\gamma_{2}\left(z, \underline{f}_{2}(z)\right)}\right|<\infty .
$$

Thus, when $\tau_{1}<\theta_{1}^{(1, \mathrm{r})}$ bounds (6.18) and (6.17) imply (6.11) with $\epsilon=\epsilon_{1}$. When $\tau_{1}>\theta_{1}^{(1, \mathrm{r})}$, Lemma 6.5 implies that

$$
\sup _{\theta_{1}^{(1, \min )} \leq \Re z \leq \theta_{1}^{(1, \max )}}\left|\frac{\gamma_{1}\left(z, \underline{f}_{2}(z)\right)}{\gamma_{2}\left(z, \underline{f}_{2}(z)\right)}\right|<\infty .
$$

This time, bounds (6.19) and (6.17) imply (6.11) with $\epsilon=\epsilon_{1}$. This completes the proof of the lemma.

Similarly, the following lemma classify the singularity of $\varphi_{1}(z)$ at $z=\tau_{2}$ when $\tau_{2}<\theta_{2}^{(2, \max )}$. Its proof is analogous to the proof of Lemma 6.6, and is omitted.

Lemma 6.7. Assume that $\tau_{2}<\theta_{2}^{(2, \max )}$. (a) In Categories I and II, $\tau_{2}=\theta_{2}^{(2, \mathrm{r})}$ and $\varphi_{1}(z)$ has a simple pole at $z=\tau_{2}$. (b) In Category III, $\varphi_{1}(z)$ has a simple pole at $z=\tau_{2}$ if $\tau_{2} \neq \theta_{2}^{(2, r)}$. It has a double pole at $z=\tau_{2}$ if $\tau_{2}=\theta_{2}^{(2, \mathrm{r})}$. (c) There exists an $\epsilon>0$ such that for each $a>0$,

$$
\sup _{\substack{z \notin B a\left(\tau_{2}\right) \\ \Re z \leq \tau_{2}+\epsilon}}\left|\varphi_{1}(z)\right|<\infty .
$$

The following lemma shows that $\varphi_{2}(z)$ is singular at $\tau_{1}$ when $\tau_{1}=\theta_{1}^{(1, \max )}$.

Lemma 6.8. Assume that $\tau_{1}=\theta_{1}^{(1, \max )}$. (a) $\varphi_{2}(z)$ is analytic in $\mathcal{G}_{\delta}\left(\theta_{1}^{(1, \max )}\right)$, where $\delta \in[0, \pi / 2)$ is chosen in Lemma 6.3. (b) For each $a>0$,

$$
\sup _{\substack{z \in \mathcal{G}_{\delta}\left(\theta_{1}^{(1, \max )}\right) \\ z \notin B_{a}\left(\theta_{1}^{(1, \mathrm{r})}\right)}}\left|\varphi_{2}(z)\right|<\infty .
$$


(c) In Category I, if $\theta^{(1, \mathrm{r})}=\theta^{(1, \max )}$, then

$$
\lim _{\substack{z \rightarrow \theta_{1}^{(1, \max )}, z \in \mathcal{G}_{\delta}\left(\theta_{1}^{(1, \max )}\right)}}\left(\theta_{1}^{(1, \max )}-z\right)^{\frac{1}{2}} \varphi_{2}(z) \neq 0,
$$

and, if $\theta^{(1, \mathrm{r})} \neq \theta^{(1, \max )}$, then

$$
\lim _{\substack{z \rightarrow \theta_{1}^{(1, \max )} \\ z \in \mathcal{G}_{\delta}\left(\theta_{1}^{(1, \max )}\right)}} \frac{\varphi_{2}(z)-\varphi_{2}\left(\theta_{1}^{(1, \max )}\right)}{\left(\theta_{1}^{(1, \max )}-z\right)^{\frac{1}{2}}} \neq 0 .
$$

(d) In Category II, if $\theta^{(1, \mathrm{r})}=\theta^{(1, \max )}$, then

$$
\lim _{\substack{z \rightarrow \theta_{1}^{(1, \max )} \\ z \in \mathcal{G}_{\delta}\left(\theta_{1}^{(1, \max )}\right)}}\left(\theta_{1}^{(1, \max )}-z\right) \varphi_{2}(z) \neq 0,
$$

and, if $\theta^{(1, \mathrm{r})} \neq \theta^{(1, \max )}$, then

$$
\lim _{\substack{z \rightarrow \theta_{1}^{(1, \max )} \\ z \in \mathcal{G}_{\delta}\left(\theta_{1}^{(1, \max )}\right)}}\left(\theta_{1}^{(1, \max )}-z\right)^{\frac{1}{2}}\left(\varphi_{2}(z)-\varphi_{2}\left(\theta_{1}^{(1, \max )}\right)\right) \neq 0 .
$$

REMARK 6.2. Limits in (6.20)-(6.23) show that $\varphi_{2}(z)$ must be singular at $z=\theta_{1}^{(1, \max )}$. Limits (6.20), (6.21) (6.23) suggest a branch-type singularity at $z=\theta_{1}^{(1, \max )}$, whereas limit (6.22) suggests a pole-type singularity at $z=\theta_{1}^{(1, \max )}$. We have not attempted to characterize the exact nature of the singularity at $z=\theta_{1}^{(1, \max )}$ for each case.

Proof. Assume that $\tau_{1}=\theta_{1}^{(1, \max )}$. We first prove that $\varphi_{2}(z)$ is analytic on $z \in \mathcal{G}_{\delta}\left(\tau_{1}\right)$, where $\delta \in[0, \pi / 2)$ is the constant in Lemma 6.3. Because $\varphi_{2}(z)$ is always analytic for $\Re z<\tau_{1}$ and $\tau_{1}>0>\theta_{1}^{(1, \min )}$, it is sufficient to prove $\varphi_{2}(z)$ is analytic for $z \in \mathcal{G}_{\delta}\left(\theta_{1}^{(1, \max )}\right) \cap\left\{z \in \mathbb{C}: \Re z>\theta_{1}^{(1, \mathrm{~min})}\right\}$. Because $\underline{f}_{2}(z)$ is analytic and $\Re f_{2}(z) \leq \theta_{2}^{(1, \max )}$ on $\mathcal{G}_{\delta}\left(\theta_{1}^{(1, \max )}\right) \cap\{z \in \mathbb{C}$ : $\left.\Re z>\theta_{1}^{(1, \text { min })}\right\}$ by Lemma $6.3, \varphi_{1}\left(\underline{f}_{2}(z)\right)$ is analytic on $\mathcal{G}_{\delta}\left(\theta_{1}^{(1, \max )}\right) \cap\{z \in \mathbb{C}:$ $\left.\Re z>\theta_{1}^{(1, \min )}\right\}$. Because $\tau_{1}=\theta_{1}^{(1, \max )}$, we have $\gamma_{2}\left(\theta^{(1, \max )}\right) \leq 0$. Therefore, by Lemma $6.5, \gamma_{2}(z, \underline{f}(z)) \neq 0$ on $\mathcal{G}_{\delta}\left(\theta_{1}^{(1, \max )}\right) \cap\left\{z: \Re z>\theta_{1}^{(1, \min )}\right\}$. Hence, the right side of $(6.8)$ is analytic on $\mathcal{G}_{\delta}\left(\theta_{1}^{(1, \max )}\right) \cap\left\{z \in \mathbb{C}: \Re z>\theta_{1}^{(1, \max )}\right\}$. The bound (6.8) follows from (6.15) and Lemma 6.3. 
We next prove (6.20) and (6.21). Indeed we now prove the following. If $\theta^{(1, \mathrm{r})}=\theta^{(1, \max )}$, then

$$
\lim _{\substack{z \rightarrow \theta_{1}^{(1, \max )}, z \in \mathcal{G}_{\delta}\left(\theta_{1}^{(1, \max )}\right)}}\left(\theta_{1}^{(1, \max )}-z\right)^{\frac{1}{2}} \varphi_{2}(z)=\frac{\Sigma_{22} \gamma_{1}\left(\theta^{(1, \max )}\right) \varphi_{1}\left(\theta_{2}^{(1, \max )}\right)}{r_{22} \sqrt{\operatorname{det}(\Sigma)\left(\theta_{1}^{(1, \max )}-\theta_{2}^{(2, \max )}\right)}}
$$

and, if $\theta^{(1, \mathrm{r})} \neq \theta^{(1, \max )}$, then

$$
\begin{aligned}
& \lim _{\substack{z \rightarrow \theta_{1}^{(1, \max )}, z \in \mathcal{G}_{\delta}\left(\theta_{1}^{(1, \max )}\right)}} \frac{\varphi_{2}(z)-\varphi_{2}\left(\theta_{1}^{(1, \max )}\right)}{\left(\theta_{1}^{(1, \max )}-z\right)^{\frac{1}{2}}} \\
& \quad=\frac{\gamma_{1}\left(\theta^{(1, \max )}\right)}{\gamma_{2}\left(\theta^{(1, \max )}\right) \Sigma_{22}} \varphi_{1}^{\prime}\left(\theta_{2}^{(1, \max )}\right) \sqrt{\operatorname{det}(\Sigma)\left(\theta_{1}^{(1, \max )}-\theta_{2}^{(2, \max )}\right) .}
\end{aligned}
$$

Here, $\varphi_{1}^{\prime}\left(\theta_{2}\right)$ is the derivative of $\varphi_{1}\left(\theta_{2}\right)$ and we have used the fact that $\varphi_{1}^{\prime}(z)$ is well defined for $z \in \mathbb{C}$ with $\Re z<\tau_{2}$, and $\varphi_{1}^{\prime}(z) \rightarrow \varphi_{1}^{\prime}\left(\theta_{2}^{(1, \max )}\right)$ as $z \rightarrow \theta_{2}^{(1, \max )}=\underline{f}_{2}\left(\theta_{1}^{(1, \max )}\right)$.

To prove $(6.24)$ and $(6.25)$, we first note that $\varphi_{1}\left(\theta_{2}^{(1, \max )}\right)<\infty$ by the assumption $\theta_{2}^{(1, \max )}<\tau_{2}$ and Lemma 5.1. Since $\underline{f}_{2}\left(\theta_{1}^{(1, \max )}\right)=\theta_{2}^{(1, \max )}$, it is easy to see from the definition of $\underline{f}_{2}$ that, for $z \in \mathcal{G}_{\delta}\left(\theta_{1}^{(1, \max )}\right) \cap\{z \in \mathbb{C} ; \Re z>$ $\left.\theta_{1}^{(1, \min )}\right\}$

$$
\begin{aligned}
& \underline{f}_{2}(z)-\theta_{2}^{(1, \max )}=\frac{\Sigma_{12}}{\Sigma_{22}}\left(\theta_{1}^{(1, \max )}-z\right)-\frac{\sqrt{\operatorname{det}(\Sigma)}}{\Sigma_{22}} \times \\
& \left|\left(\theta_{1}^{(1, \max )}-z\right)\left(z-\theta_{1}^{(1, \min )}\right)\right|^{\frac{1}{2}} \exp \left(i \frac{\omega_{-}(z)+\omega_{+}(z)}{2}\right),
\end{aligned}
$$

We also note that

$$
\begin{aligned}
\gamma_{2}\left(z, \underline{f}_{2}(z)\right) & =r_{12} z+r_{22} \underline{f}_{2}(z) \\
& =r_{12} z+r_{22} \bar{\theta}_{2}^{(1, \max )}+r_{22}\left(\underline{f}_{2}(z)-\theta_{2}^{(1, \max )}\right) \\
& =\gamma_{2}\left(z, \theta_{2}^{(1, \max )}\right)+r_{22}\left(\underline{f}_{2}(z)-\theta_{2}^{(1, \max )}\right) .
\end{aligned}
$$

Hence, if $\theta^{(1, \mathrm{r})}=\theta^{(1, \max )}$, then $\gamma_{2}\left(z, \theta_{2}^{(1, \max )}\right)=r_{12}\left(z-\theta_{1}^{(1, \max )}\right)$, which linearly vanishes as $z \rightarrow \theta_{1}^{(1, \max )}$. Therefore we have (6.24) from (6.8) and (6.26). If $\theta^{(1, \mathrm{r})} \neq \theta^{(1, \max )}$, then $\gamma_{2}\left(z, \theta_{2}^{(1, \max )}\right)$ converges to $\gamma_{2}\left(\theta^{(1, \max )}\right) \neq 0$ as $z \rightarrow \theta_{1}^{(1, \max )}$. Thus, we need to consider $\varphi_{2}(z)-\varphi_{2}\left(\theta_{1}^{(1, \max )}\right)$, which vanishes 
as $z \rightarrow \theta_{1}^{(1, \max )}$. In this case, we have

$$
\begin{aligned}
& \varphi_{2}(z)-\varphi_{2}\left(\theta_{1}^{(1, \max )}\right)=-\frac{\gamma_{1}\left(z, \underline{f}_{2}(z)\right) \varphi_{1}\left(\underline{f}_{2}(z)\right)}{\gamma_{2}\left(z, \underline{f}_{2}(z)\right)}+\frac{\gamma_{1}\left(\theta^{(1, \max )}\right) \varphi_{1}\left(\theta_{2}^{(1, \max )}\right)}{\gamma_{2}\left(\theta^{(1, \max )}\right)} \\
&=-\frac{1}{\gamma_{2}\left(z, \underline{f}_{2}(z)\right) \gamma_{2}\left(\theta^{(1, \max )}\right)}\left[\left(\gamma_{1}\left(z, \underline{f}_{2}(z)\right)-\gamma_{1}\left(\theta^{(1, \max )}\right)\right) \times\right. \\
& \varphi_{1}\left(\underline{f}_{2}(z)\right) \gamma_{2}\left(\theta^{(1, \max )}\right) \\
&+\gamma_{1}\left(\theta^{(1, \max )}\right)\left(\varphi_{1}\left(\underline{f}_{2}(z)\right)-\varphi_{1}\left(\theta_{2}^{(1, \max )}\right)\right) \gamma_{2}\left(\theta^{(1, \max )}\right) \\
&\left.-\gamma_{1}\left(\theta^{(1, \max )}\right) \varphi_{1}\left(\underline{f}_{2}\left(\theta_{1}^{(1, \max )}\right)\right)\left(\gamma_{2}\left(z, \underline{f}_{2}(z)\right)-\gamma_{2}\left(\theta^{(1, \max )}\right)\right)\right] .
\end{aligned}
$$

Thus,

$$
\begin{aligned}
& \lim _{\substack{z \rightarrow \theta_{1}^{(1, \max )}, z \in \mathcal{G}_{\delta}\left(\theta_{1}^{(1, \max )}\right)}} \frac{\varphi_{2}(z)-\varphi_{2}\left(\theta_{1}^{(1, \max )}\right)}{\left(\theta_{1}^{(1, \max )}-z\right)^{\frac{1}{2}}} \\
& =-\frac{\gamma_{1}\left(\theta^{(1, \max )}\right)}{\gamma_{2}\left(\theta^{(1, \max )}\right)} \lim _{\substack{z \rightarrow \theta_{1}^{(1, \max )}, z \in \mathcal{G}_{\delta}\left(\theta^{(1, \max )}\right)}} \frac{\varphi_{1}\left(\underline{f}_{2}(z)\right)-\varphi_{1}\left(\theta_{2}^{(1, \max )}\right)}{\left(\theta_{1}^{(1, \max )}-z\right)^{\frac{1}{2}}} \\
& =-\frac{\gamma_{1}\left(\theta^{(1, \max )}\right)}{\gamma_{2}\left(\theta^{(1, \max )}\right)} \varphi_{1}^{\prime}\left(\theta_{2}^{(1, \max )}\right) \lim _{\substack{z \rightarrow \theta_{1}^{(1, \max )} \\
z \in \mathcal{G}_{\delta}\left(\theta^{(1, \max )}\right)}} \frac{f_{2}(z)-\theta_{2}^{(1, \max )}}{\left(\theta_{1}^{(1, \max )}-z\right)^{\frac{1}{2}}} \\
& =\frac{\gamma_{1}\left(\theta^{(1, \max )}\right)}{\gamma_{2}\left(\theta^{(1, \max )}\right) \Sigma_{22}} \varphi_{1}^{\prime}\left(\theta_{2}^{(1, \max )}\right) \sqrt{\operatorname{det}(\Sigma)\left(\theta_{1}^{(1, \max )}-\theta_{2}^{(2, \max )}\right)} .
\end{aligned}
$$

Thus, we have obtained (6.25).

Now we prove (6.22) and (6.23). Assume Category II and $\tau_{1}=\theta_{1}^{(1, \max )}$. We actually prove the following. If $\theta^{(1, \mathrm{r})}=\theta^{(1, \max )}$, then

$$
\lim _{\substack{z \rightarrow \theta_{1}^{(1, \max )}, z \in \mathcal{G}_{\delta}\left(\theta_{1}^{(1, \max )}\right)}}\left(\theta_{1}^{(1, \max )}-z\right) \varphi_{2}(z)=\frac{\Sigma_{22}^{2} \gamma_{1}\left(\theta^{(1, \max )}\right) \gamma_{2}\left(\theta^{(2, \mathrm{r})}\right) \varphi_{2}\left(\theta_{1}^{(2, \mathrm{r})}\right)}{A \operatorname{det}(\Sigma)\left(\theta_{1}^{(1, \max )}-\theta_{2}^{(2, \max )}\right)}
$$

and if $\theta^{(1, \mathrm{r})} \neq \theta^{(1, \max )}$, then

$$
\begin{aligned}
\lim _{\substack{z \rightarrow \theta_{1}^{(1, \max )} \\
z \in \mathcal{G}_{\delta}\left(\theta_{1}^{(1, \max )}\right)}}\left(\theta_{1}^{(1, \max )}-z\right)^{\frac{1}{2}} \varphi_{2}(z) \\
=\frac{\Sigma_{22} \gamma_{1}\left(\theta^{(1, \max )}\right) \gamma_{2}\left(\theta^{(2, \mathrm{r})}\right) \varphi_{2}\left(\theta_{1}^{(2, \mathrm{r})}\right)}{A \gamma_{2}\left(\theta^{(1, \max )}\right) \sqrt{\operatorname{det}(\Sigma)\left(\theta_{1}^{(1, \max )}-\theta_{2}^{(2, \max )}\right)}}
\end{aligned}
$$


where

$$
A=r_{21}-\frac{\mu_{2}+\theta_{1}^{(2, \mathrm{r})} \Sigma_{12}+\theta_{2}^{(2, \mathrm{r})} \Sigma_{22}}{\mu_{1}+\theta_{2}^{(2, \mathrm{r})} \Sigma_{21}+\theta_{1}^{(2, \mathrm{r})} \Sigma_{11}} r_{11}
$$

To prove (6.27) and (6.28), we assume that $\tau_{1}=\theta_{1}^{(1, \max )}$. In this case, $\tau_{2}=\theta_{2}^{(1, \max )}=\underline{f}_{2}\left(\tau_{1}\right)$. By Category II assumption, $\left(\underline{f}_{1}\left(\tau_{2}\right), \tau_{2}\right)=\theta^{(2, \mathrm{r})}$. In particular,

$$
\underline{f}_{1}\left(\tau_{2}\right)=\underline{f}_{1}\left(\underline{f}_{2}\left(\tau_{1}\right)\right)=\theta_{1}^{(2, r)}<\theta_{1}^{(1, \max )}=\tau_{1} .
$$

Substituting (6.9) into (6.8), we have

$$
\varphi_{2}(z)=\frac{\gamma_{1}\left(z, \underline{f}_{2}(z)\right)}{\gamma_{2}\left(z, \underline{f}_{2}(z)\right)} \frac{\gamma_{2}\left(\underline{f}_{1}\left(\underline{f}_{2}(z)\right), \underline{f}_{2}(z)\right)}{\gamma_{1}\left(\underline{f}_{1}\left(\underline{f}_{2}(z)\right), \underline{f}_{2}(z)\right)} \varphi_{2}\left(\underline{f}_{1}\left(\underline{f}_{2}(z)\right)\right)
$$

for $\Re z<\tau_{1}$ as long as the denominators are nonzero. One can check that $\gamma_{2}\left(z, \underline{f}_{2}(z)\right) \neq 0$ and $\gamma_{1}\left(\underline{f}_{1}\left(\underline{f}_{2}(z)\right), \underline{f}_{2}(z)\right) \neq 0$ for $z \in \mathcal{G}_{\delta}\left(\tau_{1}\right) \cap\left\{\Re z \geq \tau_{1}\right\}$. It follows from (6.29) that $\varphi_{2}(z)$ can be analytically extendable to $z \in$ $\mathcal{G}_{\delta}\left(\tau_{1}\right) \cap\left\{\Re z \geq \tau_{1}\right\}$. Because $\left(\underline{f}_{1}\left(\underline{f}_{2}\left(\tau_{1}\right)\right), \underline{f}_{2}\left(\tau_{1}\right)\right)=\left(\underline{f}_{1}\left(\tau_{2}\right), \tau_{2}\right)=\theta^{(2, \mathrm{r})}$ and $\left(\tau_{1}, \underline{f}_{2}\left(\tau_{1}\right)\right)=\theta^{(1, \max )}=\theta^{(1, \mathrm{r})}$,

$$
\begin{array}{rlrl}
\gamma_{1}\left(\underline{f}_{1}\left(\tau_{2}\right), \tau_{2}\right) & =\gamma_{1}\left(\theta^{(2, \mathrm{r})}\right)=0, & \gamma_{2}\left(\underline{f}_{1}\left(\tau_{2}\right), \tau_{2}\right)=\gamma_{2}\left(\theta^{(2, \mathrm{r})}\right)>0, \\
\gamma_{2}\left(\tau_{1}, \underline{f}_{2}\left(\tau_{1}\right)\right)=\gamma_{2}\left(\theta^{(1, \mathrm{r})}\right)=0, & \gamma_{1}\left(\tau_{1}, \underline{f}_{2}\left(\tau_{1}\right)\right)=\gamma_{1}\left(\theta^{(1, \mathrm{r})}\right)>0 .
\end{array}
$$

Now we evaluate the limit in (6.27). Because

$$
\lim _{\substack{z \rightarrow \theta_{1}^{(1, \max )} \\ z \in \mathcal{G}_{\delta}\left(\theta_{1}^{(1, \max )}\right)}} \varphi_{2}\left(\underline{f}_{1}\left(\underline{f}_{2}(z)\right)\right)=\varphi_{2}\left(\theta_{1}^{(2, \mathrm{r})}\right)<\infty,
$$

it suffices to evaluate the limit

$$
\begin{aligned}
& \lim _{\substack{z \rightarrow \theta_{1}^{(1, \max )}, z \in \mathcal{G}_{\delta}\left(\theta_{1}^{(1, \max )}\right)}} \frac{\gamma_{2}\left(z, \underline{f}_{2}(z)\right) \gamma_{1}\left(\underline{f}_{1}\left(\underline{f}_{2}(z)\right), \underline{f}_{2}(z)\right)}{\left(\theta_{1}^{(1, \max )}-z\right)} \\
& =\lim _{\substack{z \rightarrow \theta_{1}^{(1, \max )}, z \in \mathcal{G}_{\delta}\left(\theta_{1}^{(1, \max )}\right)}} \frac{\gamma_{2}\left(z, \underline{f}_{2}(z)\right)}{\left(\theta_{1}^{(1, \max )}-z\right)^{1 / 2}} \lim _{\substack{z \rightarrow \theta_{1}^{(1, \max )}, z \in \mathcal{G}_{\delta}\left(\theta_{1}^{(1, \max )}\right)}} \frac{\gamma_{1}\left(\underline{f}_{1}\left(\underline{f}_{2}(z)\right), \underline{f}_{2}(z)\right)}{\left(\theta_{1}^{(1, \max )}-z\right)^{1 / 2}} .
\end{aligned}
$$

Because

$$
\gamma_{2}\left(z, \underline{f}_{2}(z)\right)=\left(z-\theta_{1}^{(1, \mathrm{r})}\right) r_{12}+\left(\underline{f}_{2}(z)-\theta_{2}^{(1, \mathrm{r})}\right) r_{22}
$$


we have

$$
\begin{aligned}
\lim _{\substack{z \rightarrow \theta_{1}^{(1, \max )}, z \in \mathcal{G}_{\delta}\left(\theta_{1}^{(1, \max )}\right)}} \frac{\gamma_{2}\left(z, \underline{f}_{2}(z)\right)}{\left(\theta_{1}^{(1, \max )}-z\right)^{1 / 2}} \\
=\lim _{\substack{z \rightarrow \theta_{1}^{(1, \max )}, z \in \mathcal{G}_{\delta}\left(\theta_{1}^{(1, \max )}\right)}} \frac{\left(z-\theta_{1}^{(1, \mathrm{r})}\right) r_{12}+\left(\underline{f}_{2}(z)-\theta_{2}^{(1, \mathrm{r})}\right) r_{22}}{\left(\theta_{1}^{(1, \max )}-z\right)^{1 / 2}} \\
=r_{22} \sqrt{\operatorname{det}(\Sigma)\left(\theta_{1}^{(1, \max )}-\theta_{2}^{(2, \max )}\right)} \neq 0 .
\end{aligned}
$$

Similarly, because

$$
\gamma_{1}\left(\underline{f}_{1}\left(\underline{f}_{2}(z)\right), \underline{f}_{2}(z)\right)=\left(\underline{f}_{1}\left(\underline{f}_{2}(z)\right)-\theta_{1}^{(2, \mathrm{r})}\right) r_{11}+\left(\underline{f}_{2}(z)-\theta_{2}^{(2, \mathrm{r})}\right) r_{21},
$$

we have

$$
\begin{aligned}
& \lim _{\substack{z \rightarrow \theta_{1}^{(1, \max )}, z \in \mathcal{G}_{\delta}\left(\theta_{1}^{(1, \max )}\right)}} \frac{\gamma_{1}\left(\underline{f}_{1}\left(\underline{f}_{2}(z)\right), \underline{f}_{2}(z)\right)}{\left(\theta_{1}^{(1, \max )}-z\right)^{\frac{1}{2}}} \\
& =\lim _{\substack{z \rightarrow \theta_{1}^{(1, \max )}, z \in \mathcal{G}_{\delta}\left(\theta_{1}^{(1, \max )}\right)}} \frac{\left(\underline{f}_{1}\left(\underline{f}_{2}(z)\right)-\theta_{1}^{(2, \mathrm{r})}\right) r_{11}+\left(\underline{f}_{2}(z)-\theta_{2}^{(2, \mathrm{r})}\right) r_{21}}{\left(\theta_{1}^{(1, \max )}-z\right)^{\frac{1}{2}}} \\
& =\lim _{\substack{z \rightarrow \theta_{1}^{(1, \max )}, z \in \mathcal{G}_{\delta}\left(\theta_{1}^{(1, \max )}\right)}} \frac{\left(\underline{f}_{1}^{\prime}\left(\tau_{2}\right) r_{11}+r_{21}\right)\left(\underline{f}_{2}(z)-\theta_{2}^{(2, \mathrm{r})}\right)}{\left(\theta_{1}^{(1, \max )}-z\right)^{\frac{1}{2}}} \\
& =\left(\underline{f}_{1}^{\prime}\left(\tau_{2}\right) r_{11}+r_{21}\right) \sqrt{\operatorname{det}(\Sigma)\left(\theta_{1}^{(1, \max )}-\theta_{2}^{(2, \max )}\right)},
\end{aligned}
$$

where

$$
r_{11} \underline{f}_{1}^{\prime}\left(\tau_{2}\right)+r_{21}=r_{21}-\frac{\mu_{2}+\theta_{1}^{(2, \mathrm{r})} \Sigma_{12}+\theta_{2}^{(2, \mathrm{r})} \Sigma_{22}}{\mu_{1}+\theta_{2}^{(2, \mathrm{r})} \Sigma_{21}+\theta_{1}^{(2, \mathrm{r})} \Sigma_{11}} r_{11},
$$

which cannot vanish because the line $\gamma_{2}(\theta)=0$ is not tangent to the ellipse $\gamma(\theta)=0$ at $\theta^{(2, \mathrm{r})}$. This proves $(6.27)$.

Now we assume that $\theta^{(1, \mathrm{r})} \neq \theta^{(1, \max )}$. In that case, $\left(\underline{f}_{1}\left(\underline{f}_{2}\left(\tau_{1}\right)\right), \underline{f}_{2}\left(\tau_{1}\right)\right)=$ $\left(\underline{f}_{1}\left(\tau_{2}\right), \tau_{2}\right)=\theta^{(2, \mathrm{r})}$, but $\left(\tau_{1}, \underline{f}_{2}\left(\tau_{1}\right)\right)=\theta^{(1, \max )} \neq \theta^{(1, \mathrm{r})}$, thus $\gamma_{2}\left(\tau_{1}, \underline{f}_{2}\left(\tau_{1}\right)\right) \neq$ 0 . Now (6.28) follows from the proof of (6.27). 
7. Proofs of the theorems. In this section, we prove Theorems 2.1, 2.2 and 2.3. The ideas of proofs have the same spirit as those in [26] (see also [17]), but we need more ideas. The proofs use the analytic extensions of complex variable functions developed in Section 6, complex inversion techniques in Appendix $\mathrm{C}$, and decompositions of moment generating functions in Appendix D.

7.1. The proof of Theorem 2.1. The next lemma partially characterizes the boundary of the domain $\mathcal{D}$.

Lemma 7.1. For any $\theta \in \mathbb{R}^{2}, \varphi(\theta)=\infty$ if either $\theta_{1}>\tau_{1}$ or $\theta_{2}>\tau_{2}$.

ProOF. By the symmetric role of the coordinates, we only need to prove that $\theta_{1}>\tau_{1}$ implies $\varphi(\theta)=\infty$. Since $\tau_{1} \leq \theta_{1}^{(1, \max )}$, either $\tau_{1}<\theta_{1}^{(1, \max )}$ or $\tau_{1}=\theta_{1}^{(1, \max )}$ holds. We separately consider these two cases. First consider the case where $\tau_{1}<\theta_{1}^{(1, \max )}$. In this case, $\tau_{1}=\theta_{1}^{(1, \mathrm{r})}$ for Category I or Category III, and $\tau_{1}=\underline{f}_{1}\left(\theta_{2}^{(2, \mathrm{r})}\right)$ for Category II. When $\tau_{1}=\theta_{1}^{(1, \mathrm{r})}$, we have $\varphi(\theta)=\infty$ for $\theta_{1}>\tau_{1}$, by Lemma 5.2. Otherwise, we have Category II. Since $\tau_{1}<\theta_{1}^{(1, \max )}, \varphi_{2}(z)$ has a pole at $z=\tau_{1}$ by Lemma 6.6. This implies $\varphi_{2}\left(\theta_{1}\right)=\infty$ for $\theta_{1}>\tau_{1}$, so $\varphi(\theta)=\infty$ for $\theta_{1}>\tau_{1}$ by (b) of Lemma 4.1.

We next consider the case where $\tau_{1}=\theta_{1}^{(1, \max )}$. By Lemma $6.8, \varphi_{2}(z)$ is singular at $z=\tau_{1}$. Hence, $\varphi_{2}\left(\theta_{1}\right)=\infty$ if $\theta_{1}>\tau_{1}$. This implies $\varphi(\theta)=\infty$ by (b) of Lemma 4.1 .

We are now ready to prove Theorem 2.1. By Lemma 5.1, $\varphi_{2}\left(\theta_{1}\right)<\infty$ and $\varphi_{2}\left(\theta_{1}\right)<\infty$ for $\theta<\tau$, and hence, $\varphi(\theta)<\infty$ for $\theta \in\left\{\eta \in \mathbb{R}^{2}: \eta<\tau\right\} \cap \Gamma_{\max }$ by part (c) of Lemma 4.1. We claim that $\varphi(\theta)=\infty$ for $\theta<\tau$ and $\theta \notin \bar{\Gamma}_{\text {max }}$ (see Figure 12), where $\bar{\Gamma}_{\max }$ is the closure of $\Gamma_{\max }$. If this claim holds true, we have Theorem 2.1 by Lemma 7.1.

We first note that, if $\tau \in \bar{\Gamma}$, there is no $\theta$ such that $\theta<\tau$ and $\theta \notin \bar{\Gamma}_{\text {max }}$. Hence, we can assume that $\tau \notin \bar{\Gamma}$. We then must be in Category I and $\eta_{1}^{(2)}<\eta_{1}^{(1)}$, where $\eta^{(k)}$ is defined in (2.11); see also Figure 12. Let

$$
\partial \Gamma^{+}=\left\{\left(\theta_{1}, \bar{f}_{2}\left(\theta_{1}\right)\right): \theta_{1} \in\left(\eta_{1}^{(2)}, \eta_{1}^{(1)}\right)\right\}
$$

be the segment of the ellipse $\partial \Gamma$ that is within both the upper half and the right half of the ellipse and is below $\tau$. In Figure $12, \partial \Gamma^{+}$is the piece of the ellipse that borders the shaded region. To prove that claim, it suffices to prove that for each relatively open neighborhood $B \subset \partial \Gamma^{+}$, there exists a $\theta \in B$ such that $\varphi(\theta)=\infty$ because $\tilde{\theta} \geq \theta$ implies $\varphi(\tilde{\theta})=\infty$. 


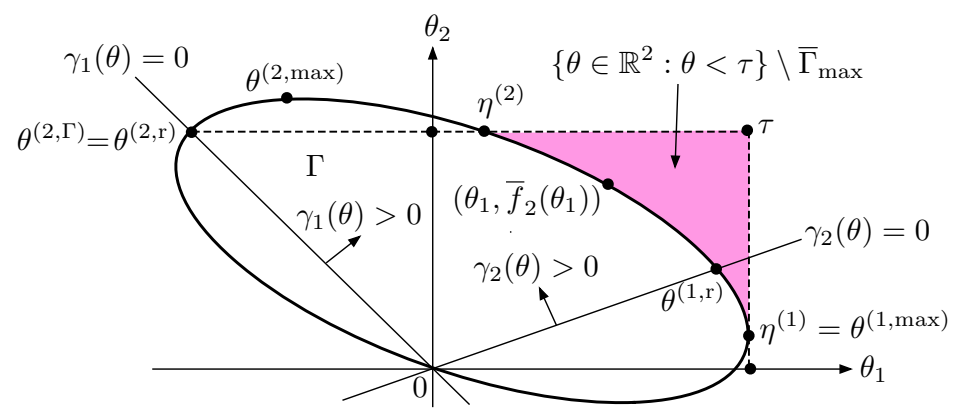

FIG 12. The area of $\left\{\theta \in \mathbb{R}^{2}: \theta<\tau\right\} \backslash \bar{\Gamma}_{\max }$

Assume on the contrary that there exists a relatively open neighborhood

$$
B=\left\{\left(\theta_{1}, \bar{f}_{2}\left(\theta_{1}\right)\right): \theta_{1} \in\left(b_{1}, b_{2}\right)\right\} \subset \partial \Gamma^{+}
$$

with $\eta_{1}^{(2)}<b_{1}<b_{2}<\eta_{1}^{(1)}$ such that $\varphi(\theta)<\infty$ for each $\theta \in B$. Then, it follows from (a) and (b) of Lemma 4.1 that

$$
\gamma_{2}\left(\theta_{1}, \bar{f}_{2}\left(\theta_{1}\right)\right) \varphi_{2}\left(\theta_{1}\right)=-\gamma_{1}\left(\theta_{1}, \bar{f}_{2}\left(\theta_{1}\right)\right) \varphi_{1}\left(\bar{f}_{2}\left(\theta_{1}\right)\right), \quad \theta_{1} \in\left(b_{1}, b_{2}\right) .
$$

We analytically expand the domain for (7.1). For this, we replace $\theta_{1}$ by complex number $z$, and consider

$$
\gamma_{2}\left(z, \bar{f}_{2}(z)\right) \varphi_{2}(z)=-\gamma_{1}\left(z, \bar{f}_{2}(z)\right) \varphi_{1}\left(\bar{f}_{2}(z)\right)
$$

for $z$ in some domain in $\mathbb{C}$. Using similar arguments in Lemma 6.3, we can see that $\bar{f}_{2}(z)$ is analytic for $\Re z \in\left(\theta_{1}^{(1, \min )}, \theta_{1}^{(1, \max )}\right)$. Because $\bar{f}_{2}\left(\theta_{1}\right)$ is strictly decreasing in $\left(\eta_{1}^{(2)}, \eta_{1}^{(1)}\right)$, we have $\bar{f}_{2}\left(\theta_{1}\right) \leq \tau_{2}$ for each $\theta_{1} \in\left(\eta_{1}^{(2)}, \theta_{1}^{(1, \max )}\right)$. Hence, for each sufficiently small $\epsilon>0, \Re \bar{f}_{2}(z)<\tau_{2}$ for $z \in G$, where

$$
G \equiv\left\{z=x+y i \in \mathbb{C}: x \in\left(\eta_{1}^{(2)}+\epsilon, \theta_{1}^{(1, \max )}-\epsilon\right), y \in(-\epsilon, \epsilon)\right\} .
$$

Therefore, the right side of (7.2) is analytic for $z \in G$. We assume $\epsilon>$ 0 is small enough so that $\left(b_{1}, b_{2}\right) \subset\left(\eta_{1}^{(2)}+\epsilon, \eta_{1}^{(1)}-\epsilon\right)$. By (7.1) and the analytic extension of complex functions, the left side of (7.2) is analytically extendable on $G$. This implies that (7.2) holds for $z=x+y i$ with $x \in$ $\left(\eta_{1}^{(2)}+\epsilon, \eta_{1}^{(1)}-\epsilon\right)$ and $y \in(-\epsilon, \epsilon)$ since $\varphi_{2}\left(\theta_{1}\right)<\infty$ for $\theta_{1}<\tau_{1}=\eta_{1}^{(1)}$. Furthermore, if $\gamma_{2}\left(z, \bar{f}_{2}(z)\right) \neq 0$ for all $z \in G$, then $\varphi_{2}(z)$ is analytic on $G$.

Now we argue that under condition (7.1) it must be true that $\tau_{1}=\theta_{1}^{(1, \max )}$ and $\tau_{2}=\theta_{2}^{(2, \max )}$. Suppose on the contrary that $\tau_{1}<\theta_{1}^{(1, \max )}$. Then the line 
$\gamma_{2}(\theta)=0$ intersects the lower half of the ellipse, and thus $\gamma_{2}\left(z, \bar{f}_{2}(z)\right) \neq 0$ for $z \in G$. Thus, $\varphi_{2}(z)$ is analytic on $G$, and in particular at $z=\theta_{1}^{(1, \max )}-2 \epsilon$, which is larger than $\eta_{1}^{(1)}=\tau_{1}$ for $\epsilon>0$ small enough, contradicting part (b) of Lemma 5.2. Thus we have proved that $\tau_{1}=\theta_{1}^{(1, \max )}$. Exchanging the role of $\theta_{1}$ and $\theta_{2}$, we also have $\tau_{2}=\theta_{2}^{(2, \max )}$. Because $\epsilon>0$ can be arbitrarily small, we have proved that (7.2) holds for $z=\theta_{1} \in\left(\theta_{1}^{(2, \max )}, \theta_{1}^{(1, \max )}\right)$. Also, by Lemma $2.2, \theta^{(1, \mathrm{r})}$ and $\theta^{(2, \mathrm{r})}$ must be on the upper ellipse curve from $\theta^{(1, \max )}$ to $\theta^{(2, \max )}$ in such a way that $\theta^{(2, \mathrm{r})}$ is above $\theta^{(1, \mathrm{r})}$, and therefore

$$
\theta_{1}^{(2, \max )} \leq \theta_{1}^{(2, \mathrm{r})}<\theta_{1}^{(1 \mathrm{r})} \leq \theta_{1}^{(1, \max )} .
$$

Thus, (7.2) holds for $z=\theta_{1} \in\left(\theta_{1}^{(2, r}, \theta_{1}^{(1, r}\right)$, contradicting the facts that

$\gamma_{1}\left(\theta_{1}, \bar{f}_{2}\left(\theta_{1}\right)\right)>0, \quad \gamma_{2}\left(\theta_{1}, \bar{f}_{2}\left(\theta_{1}\right)\right)>0, \quad \varphi_{1}\left(\bar{f}_{2}\left(\theta_{1}\right)\right)<\infty, \quad \varphi_{2}\left(\theta_{1}\right)<\infty$

for $\theta_{1} \in\left(\theta_{1}^{(2, \mathrm{r})}, \theta_{1}^{(1, \mathrm{r})}\right)$. This completes the proof of the theorem.

7.2. The proof of Theorem 2.2. We now start to prove Theorem 2.2. From (1.9), the definition of $\alpha_{c}$ in (2.13), Lemma 6.1, and Theorem 2.1, we know that the moment generating function $\psi_{c}(z)$ for $c_{1} Z_{1}+c_{2} Z_{2}$ is analytic for $\Re z<\alpha_{c}$ and is singular at $\alpha_{c}$. Again from Theorem 2.1,

$$
\alpha_{c} \leq \min \left(\tau_{1} / c_{1}, \tau_{2} / c_{2}\right) \text {. }
$$

Thus, $\varphi_{1}\left(c_{2} z\right), \varphi_{2}\left(c_{1} z\right)$ and $\varphi\left(c_{1} z, c_{2} z\right)$ are all finite for $z \in \mathbb{C}$ with $\Re z<\alpha_{c}$. Hence, from (2.3), we have

$$
\gamma(z c) \psi_{c}(z)=\gamma_{1}(z c) \varphi_{1}\left(c_{2} z\right)+\gamma_{2}(z c) \varphi_{2}\left(c_{1} z\right) \quad \text { for } \Re z<\alpha_{c} .
$$

Recall that $z_{c}$ is the nonzero root of $\gamma(z c)=0$. Let $\zeta_{c}(z)=-\left(\frac{1}{2}\langle c, \Sigma c\rangle z+\right.$ $\langle c, \mu\rangle)$. Then, $z_{c}$ is the root of $\zeta_{c}(z)=0$, and

$$
\frac{\gamma_{k}(z c)}{\gamma(z c)}=\frac{\gamma_{k}(c)}{\zeta_{c}(z)} \quad \text { for } z \in \mathbb{C} \backslash\left\{z_{c}, 0\right\}
$$

Therefore,

$$
\psi_{c}(z)=\frac{\gamma_{1}(c) \varphi_{1}\left(c_{2} z\right)+\gamma_{2}(c) \varphi_{2}\left(c_{1} z\right)}{\zeta_{c}(z)}
$$

for $\Re z<\alpha_{c}$ and $z \neq z_{c}$, where it can be checked that (7.3) is valid for $z=0$. The numerator in the right side of (7.3) is analytic for $\Re z<\min \left(\tau_{1} / c_{1}, \tau_{2} / c_{2}\right)$ because of Lemma 6.2. Because $\psi_{c}(z)$ is analytic for $\Re z<\alpha_{c}, z=z_{c}$ must 
be a removable singularity of the right side of (7.3) if $z_{c}<\alpha_{c}$. Hence, if $z_{c}<\alpha_{c}$, we have

$$
\gamma_{1}(c) \varphi_{1}\left(c_{2} z_{c}\right)+\gamma_{2}(c) \varphi_{2}\left(c_{1} z_{c}\right)=0
$$

and therefore

$$
\psi_{c}(z)=-\gamma_{1}(c) h_{1}(z)-\gamma_{2}(c) h_{2}(z), \quad \Re z<\alpha_{c},
$$

where

$$
h_{1}(z)=\frac{\varphi_{1}\left(c_{2} z_{c}\right)-\varphi_{1}\left(c_{2} z\right)}{\zeta_{c}(z)}, \quad h_{2}(z)=\frac{\varphi_{2}\left(c_{1} z_{c}\right)-\varphi_{2}\left(c_{1} z\right)}{\zeta_{c}(z)} .
$$

If $z_{c} \geq \alpha_{c}$, from (7.3), it is easy to see that the moment generating function $\psi_{c}(z)$ can be expressed as a linear combination of the form in (D.2) with $k=1$. If $z_{c}<\alpha_{c}$, from $(7.4), \psi_{c}(z)$ can be expressed as a linear combination of the form in (D.3). By Lemmas D.2 and D.3, each term in these two linear decompositions is the moment generating function of a continuous density since $\varphi_{1}$ and $\varphi_{2}$ are the moment generating functions of the measures $\nu_{1}$ and $\nu_{2}$, respectively, and both $\nu_{1}$ and $\nu_{2}$ have densities (see $\left.[3,13]\right)$. Thus, by Lemma D.1, the distribution of $\langle c, Z(\infty)\rangle$ also has a continuous density $p_{c}(x)$ on $[0, \infty)$.

In the following proof, for many cases we actually prove that the density $p_{c}(x)$ has an exact asymptotic, which implies that the exact asymptotic for the tail distribution

$$
q_{c}(x)=\mathbb{P}(\langle c, Z(\infty)\rangle \geq x), \quad x \geq 0
$$

by Lemma D.5. However, in some cases, we are not able to establish the exact asymptotic for density $p_{c}(x)$. In these cases, we work with the moment generating function $\bar{\psi}_{c}(z)$ of the tail probability $q_{c}(x)$ directly, where

$$
\bar{\psi}_{c}(z)=\int_{0}^{\infty} e^{z x} \mathbb{P}(\langle c, Z(\infty)\rangle \geq x) d x=\int_{0}^{\infty} e^{z x} q_{c}(x) d x .
$$

One can check that

$$
\bar{\psi}_{c}(z)=\frac{\psi_{c}(z)-1}{z} \quad \text { for } \Re z<\alpha_{c}
$$

and $z=0$ is a removable singularity of $\bar{\psi}_{c}(z)$.

(a) Assume that $0 \leq \beta<\beta_{1}$. In this case $\alpha_{c}=\tau_{1} / c_{1}$. We now show that

$$
\alpha_{c}=\frac{\tau_{1}}{c_{1}}<\frac{\tau_{2}}{c_{2}} .
$$


Inequality (7.6) clearly holds when $\tau=\eta^{(1)}=\eta^{(2)}$ because $\beta<\beta_{1}$. When $\beta<\beta_{1}<\beta_{2}$, we have

$$
\frac{\tau_{1}}{c_{1}}<\frac{\bar{f}_{2}\left(\tau_{1}\right)}{c_{2}}<\frac{\tau_{2}}{c_{2}}
$$

where the second inequality follows from $\bar{f}_{2}\left(\tau_{1}\right)<\tau_{2}$ because $\beta_{1}<\beta_{2}$. Therefore, we have proved (7.6). By (7.6) and Lemma 6.2, $\varphi_{1}\left(c_{2} z\right)$ is analytic at $\alpha_{c}$.

(a.i) Assume that $\tau_{1} \neq \theta_{1}^{(1, \max )}$. In this case, Category I implies that $\theta_{2}^{(1, \mathrm{r})}<\theta_{2}^{(1, \max )}$ and $\tau_{1}=\theta_{1}^{(1, \mathrm{r})}$. We first consider the case that $\alpha_{c} c=\theta^{(1, \mathrm{r})}$. In this case, $\gamma_{2}(c)=0$ and $\alpha_{c} c \in \partial \Gamma$. Hence $z_{c}=\alpha_{c}$ and $\zeta_{c}\left(z_{c}\right)=0$. By (7.3), we have

$$
\psi_{c}(z)=\frac{\gamma_{1}(c) \varphi_{1}\left(c_{2} z\right)}{\zeta_{c}(z)}
$$

Therefore, $\psi_{c}(z)$ has a simple pole at $z=z_{c}=\alpha_{c}$. By Lemma D.4, we conclude that $p_{c}(x)$ has exact asymptotic given by

$$
f_{c}(x)=e^{-\alpha_{c} x} \quad \text { for } x \geq 0 .
$$

Now consider the case that $\alpha_{c} c \neq \theta^{(1, \mathrm{r})}$. In this case, $\gamma_{2}(c) \neq 0$ and $\gamma\left(\alpha_{c} c\right) \neq 0$. The latter is equivalent to $\zeta_{c}\left(\alpha_{c}\right) \neq 0$. Therefore, $z_{c} \neq \alpha_{c}$ and $1 / \zeta_{c}(z)$ is analytic at $z=\alpha_{c}$. We now work with $\bar{\psi}_{c}(z)$ to obtain tail asymptotic for $q_{c}(x)$. By $(7.5), \bar{\psi}_{c}(z)$ is analytic on $\Re z<\alpha_{c}$. It follows from (7.3) and (7.5) that

$$
\bar{\psi}_{c}(z)=\frac{\gamma_{1}(c) \varphi_{1}\left(c_{2} z\right)}{z \zeta_{c}(z)}+\frac{\gamma_{2}(c) \varphi_{2}\left(c_{1} z\right)}{z \zeta_{c}(z)}-\frac{1}{z} \quad \text { for } \Re z<\alpha_{c}
$$

except at $z=0$ and $z=z_{c}$ if $z_{c}<\alpha_{c}$. Since $\bar{\psi}_{c}(z)$ is analytic at points $z=0$ and $z=z_{c}$ if $z_{c}<\alpha_{c}, z=0$ and $z=z_{c}$ if $z_{c}<\alpha_{c}$ must be removable singularities of the right side of (7.9). Since $\varphi_{1}\left(c_{2} z\right)$ is analytic at $z=\alpha_{c}$, it follows from Lemma 6.6 that there exists an $0<\epsilon \leq \epsilon_{0} / c_{1}$ such that $\varphi_{1}\left(c_{2} z\right)$ and $\varphi_{2}\left(c_{1} z\right)$ are analytic in $\Re z<\alpha_{c}+2 \epsilon$ except at $z=\alpha_{c}$, where $\epsilon_{0}>0$ is the constant in Lemma 6.6. Thus, $\bar{\psi}_{c}(z)$ is analytic in $\Re z<\alpha_{c}+2 \epsilon$ except at $z=\alpha_{c}$. Furthermore, Lemma 6.6 implies that $\bar{\psi}_{c}(z)$ has a simple pole at $z=\alpha_{c}$. Thus, $\bar{\psi}_{c}(z)$ satisfies condition (C1a) of Lemma C.1 with $m=1$ and $k_{0}=1$. Next, we verify that $\bar{\psi}_{c}(z)$ satisfies condition (C1c) of Lemma C.1, namely,

$$
\int_{-\infty}^{\infty} e^{-i y x} \bar{\psi}_{c}\left(\alpha_{c}+\epsilon+i y\right) d y \quad \text { converges uniformly }
$$


for $x>T$ some $T>0$. To prove (7.10), we separately consider three integrals, each corresponding to one of the three terms on the right side of (7.9). The last integral converges uniformly following the computation on page 237 of [5]. Because of (6.11) and (6.7), there are constants $a, b>0$ such that

$$
\left|\bar{\psi}_{c}(z)+1 / z\right| \leq a /|z|^{2} \quad \text { for }|\Im z| \geq b \text { and } \Re z \leq 0 \leq \alpha_{c}+\epsilon,
$$

where $\Im z$ is the imaginary part of $z$. Hence, similar to condition (C.4), the integrals of the first two terms on the right side of (7.9) uniformly converges. Thus, (C1c) of Lemma C.1 is verified while (C1b) of the same lemma is immediate from (7.11). By Lemma C.1, $q_{c}(x)$ has exact asymptotic that is given by (7.8).

Note that when $\alpha_{c} c \neq \theta^{(1, \mathrm{r})}$, we are unable to verify condition (7.10) for $\psi_{c}(z)$, and hence not able to obtain the exact asymptotic for $p_{c}(x)$.

(a.ii) Assume that $\tau_{1}=\theta_{1}^{(1, \max )}$. In this case we apply Lemma C.2 in addition to Lemma D.4 to get exact asymptotics. Now we examine the details. Because $\tau_{1}=\theta_{1}^{(1, \max )}$, we have $z_{c}<\alpha_{c}$ and hence $\zeta_{c}\left(\alpha_{c}\right)<0$. Thus, we can use the expression (7.4) with functions $h_{1}$ and $h_{2}$ of (7.2). Because $\varphi_{1}\left(c_{2} z\right)$ is analytic at $z=\alpha_{c}, h_{1}\left(\alpha_{c}+\epsilon\right)$ is finite, for some $\epsilon>0$. By Lemma D.3, $h_{1}$ is a moment generating function of a signed measure $\xi_{1}$. Because $h_{1}\left(\alpha_{c}+\epsilon\right)$ is finite, the tail of $\xi_{1}$ decays as

$$
\xi_{1}([x, \infty))=o\left(e^{-\left(\alpha_{c}+\epsilon\right) x}\right) \quad \text { as } x \rightarrow \infty .
$$

On the other hand, the function $h_{2}$ is singular at $z=\alpha_{c}$. The analytical behavior $h_{2}(z)$ around $z=\alpha_{c}$ is identical to that of $\varphi_{2}\left(c_{1} z\right)$. We apply Lemma 6.8 to find the analytical behavior of $\varphi_{2}\left(c_{1} z\right)$ at $\alpha_{c}$. We consider two separate cases.

We first consider the case that $\eta^{(1)}=\theta^{(1, \max )} \neq \theta^{(1, \mathrm{r})}$. By part (a) of Lemma $6.8, \varphi_{2}\left(c_{1} z\right)$ is analytic on $\mathcal{G}_{\delta}\left(\alpha_{c}\right)$. Thus $h_{2}(z)$ is analytic on $\mathcal{G}_{\delta}\left(\alpha_{c}\right)$, and therefore $h_{z}(z)$ satisfies condition (C2a) of Lemma C.2. From (6.25) of Lemma 6.8 and the fact that $c_{1} \alpha_{c}=\theta_{1}^{(1, \max )}$, it follows that, for some constant $K_{1}>0$,

$$
\lim _{\substack{z \rightarrow \alpha_{c} \\ z \in \mathcal{G}_{\delta}\left(\alpha_{c}\right)}} \frac{\varphi_{2}\left(c_{1} z\right)-\varphi_{2}\left(c_{1} \alpha_{c}\right)}{\left(\alpha_{c}-z\right)^{1 / 2}}=-K_{1} .
$$

Since

$h_{2}(z)-h_{2}\left(\alpha_{c}\right)=\frac{\varphi_{2}\left(c_{1} \alpha_{c}\right)-\varphi_{2}\left(c_{1} z\right)}{\zeta_{c}(z)}+\left(\varphi_{2}\left(c_{1} z_{c}\right)-\varphi_{2}\left(c_{1} \alpha_{c}\right)\right) \frac{\zeta_{c}\left(\alpha_{c}\right)-\zeta_{c}(z)}{\zeta_{c}\left(\alpha_{c}\right) \zeta_{c}(z)}$, 
we have

$$
\lim _{\substack{z \rightarrow \alpha_{c} \\ z \in \mathcal{G}_{\delta}\left(\alpha_{c}\right)}} \frac{h_{2}(z)-h_{2}\left(\alpha_{c}\right)}{\left(\alpha_{c}-z\right)^{1 / 2}}=\frac{K_{1}}{\zeta_{c}\left(\alpha_{c}\right)}<0 .
$$

Hence, $h_{2}(z)$ satisfies conditions (C2c). One can check that condition (C2b) is also satisfied for $h_{2}(z)$ because of (6.8). Thus, applying Lemma C.2 with $\lambda=-\frac{1}{2}$, one yields that the measure for $h_{2}$ has the exact tail asymptotic $x^{-3 / 2} \exp \left(-\alpha_{c} x\right)$. Since this dominates that of $\xi_{1}$ in $(7.12)$ and $\gamma_{2}(c)<0$, by Lemma D.1, $p_{c}(x)$ has exact asymptotic

$$
f_{c}(x)=x^{-3 / 2} \exp \left(-\alpha_{c} x\right) \quad \text { for } x>0 .
$$

We next consider the case $\eta^{(1)}=\theta^{(1, \max )}=\theta^{(1, \mathrm{r})}$. This case is exactly the same as the previous one except that, for some $K_{2}>0$,

$$
\lim _{\substack{z \rightarrow \alpha_{c} \\ z \in \mathcal{G}_{\delta}\left(\alpha_{c}\right)}}\left(\alpha_{c}-z\right)^{1 / 2} \varphi_{2}\left(c_{1} z\right)=K_{2}
$$

by Lemma 6.8. Since this implies

$$
\lim _{\substack{z \rightarrow \alpha_{c} \\ z \in \mathcal{G}_{\delta}\left(\alpha_{c}\right)}}\left(\alpha_{c}-z\right)^{1 / 2} h_{2}(z)=\frac{-K_{2}}{\zeta_{c}\left(\alpha_{c}\right)}>0,
$$

Lemma C.2 implies that the measure for $h_{2}$ has the exact tail asymptotic $x^{-1 / 2} \exp \left(-\alpha_{c} x\right)$. Hence, again by Lemma D.1 and (7.12), $p_{c}(x)$ has the exact asymptotic

$$
f_{c}(x)=x^{-1 / 2} \exp \left(-\alpha_{c} x\right) \quad \text { for } x>0 .
$$

(b1) Assume that $\beta=\beta_{1}<\beta_{2}$. In this case, either $\eta^{(1)} \neq \theta^{(1, \max )}, \eta^{(1)}=$ $\theta^{(1, \max )} \neq \theta^{(1, \mathrm{r})}$ or $\eta^{(1)}=\theta^{(1, \max )}=\theta^{(1, \mathrm{r})}$. Note that $\eta^{(1)}$ is above $\theta^{(1, \max )}$ and $\theta^{(1, \mathrm{r})}$ for $\eta^{(1)} \neq \theta^{(1, \max )}$. When $\alpha_{c} c=\eta^{(1)} \neq \theta^{(1, \max )}, \varphi_{2}(z)$ has a simple pole at $z=\tau_{1}$ and thus $\psi_{c}(z)$ has a double pole because $z_{c}=\alpha_{c}$. Therefore, by Lemmas 6.6 and C.1, $q_{c}(x)$ has the exact tail asymptotic $f_{c}(x)$ given by

$$
f_{c}(x)=x e^{-\alpha_{c} x} \quad \text { for } x \geq 0 .
$$

We now consider $\alpha_{c} c=\eta^{(1)}=\theta^{(1, \max )} \neq \theta^{(1, \mathrm{r})}$. In this case, $\zeta_{c}\left(\alpha_{c}\right)=0$ while $\gamma_{1}(c)>0$ and $\gamma_{2}(c)<0$. Since

$$
\zeta_{c}(z)=-\langle c, \Sigma c\rangle\left(z-\alpha_{c}\right),
$$


the same argument as in case (a.ii) when $0 \leq \beta<\beta_{1}$ shows that $h_{2}(z)$ is analytic in $\mathcal{G}_{\delta}\left(\alpha_{c}\right)$, condition $(\mathrm{C} 2 \mathrm{~b})$ is satisfied for $h_{2}(z)$, and

$$
\lim _{\substack{z \rightarrow \alpha_{c} \\ z \in \mathcal{G}_{\delta}\left(\alpha_{c}\right)}}\left(\alpha_{c}-z\right)^{1 / 2} h_{2}(z)=-\frac{\gamma_{2}(c)}{\langle c, \Sigma c\rangle} \lim _{\substack{z \rightarrow \alpha_{c} \\ z \in \mathcal{G}_{\delta}\left(\alpha_{c}\right)}} \frac{\varphi_{2}\left(c_{1} z\right)-\varphi_{2}\left(c_{1} \alpha_{c}\right)}{\left(\alpha_{c}-z\right)^{1 / 2}} .
$$

Applying Lemma C.2 with $\lambda=1 / 2$, we have that $p_{c}(x)$ has the exact tail asymptotic $f_{c}(x)$ given by $(7.14)$.

We consider the remaining case that $\alpha_{c} c=\eta^{(1)}=\theta^{(1, \max )}=\theta^{(1, \mathrm{r})}$. In this case, we have representation (7.7), and therefore $\psi_{c}(z)$ has a simple pole at $\alpha_{c}$. Hence, the proof is identical to case (a.i) when $\alpha_{c} c=\theta^{(1, \mathrm{r})}$, and $p_{c}(x)$ has exact asymptotic given by (7.8).

(b2) Assume $\beta=\beta_{1}=\beta_{2}$. In this case $\eta^{(1)}=\eta^{(2)}=\tau, \gamma_{2}(c)>0$ and $\gamma_{1}(c)>0$. We first claim $\tau_{1}<\theta_{1}^{(1, \max )}$. Otherwise, $\tau_{1}=\theta_{1}^{(1, \max )}$, which implies that $\tau_{2}=\theta_{2}^{(1, \max )}=\theta_{2}^{(1, \Gamma)}$ and we must have Category II, leading to a contradiction. Thus, $\tau_{1}<\theta_{1}^{(1, \max )}$ and hence $\varphi_{2}\left(c_{1} z\right)$ has a simple pole at $\alpha_{c}$ by Lemma 6.6. Similarly, we can prove that $\tau_{2}<\theta_{2}^{(2, \max )}$ and hence and $\varphi_{1}\left(c_{2} z\right)$ has a simple pole at $\alpha_{c}$ by Lemma 6.7 . We consider two cases separately. When $\alpha_{c} c \notin \partial \Gamma$, we have $\zeta_{c}\left(\alpha_{c}\right)>0$. It follows from (7.9) in part (a.i) of the proof of Theorem 2.2 that $\bar{\psi}_{c}(z)$ has a simple pole at $z=\alpha_{c}$. It follows from Lemma C.1 that $q_{c}(x)$ has exact asymptotic $f_{c}(x)$ that is given by (7.8). When $\alpha_{c} c=\eta^{(1)} \in \partial \Gamma, \zeta_{c}\left(\alpha_{c}\right)=0$. It follows from (7.9) that $\bar{\psi}_{c}(z)$ has a double pole at $z=\alpha_{c}=z_{c}$. Thus, $\bar{\psi}_{c}(z)$ satisfies condition (C1a) of Lemma C. 1 for $m=1$ and $k_{0}=2$. Condition (C.4) is satisfied exactly as in the proof of (a.i). By Lemma C.1, $q_{c}(x)$ has the exact asymptotic $f_{c}(x)$ given by (7.15).

(b3) Assume that $\beta_{1}<\beta<\beta_{2}$. In this case, the ray $c$ intersects $\partial \Gamma$ at $\alpha_{c} c$. Thus, $\alpha_{c}=z_{c}<\min \left(\tau_{1} / c_{1}, \tau_{2} / c_{2}\right)$ and $\zeta_{c}\left(\alpha_{c}\right)=0$. Therefore, the numerator of (7.3) is analytic at $\alpha_{c}$. If the numerator of the right side of (7.3) vanishes at $z=\alpha_{c}$, then $z=\alpha_{c}$ is a removable singularity for $\psi_{c}$. This implies that $\psi_{c}$ is analytic at $\alpha_{c}$, leading to a contradiction. Therefore, from (7.3), $\psi_{c}(c)$ has a simple pole at $z=\alpha_{c}$. Applying Lemma D.4, we immediately see that $p_{c}(x)$ has the exact asymptotic in (7.8).

(b4) Assume that $\beta=\beta_{2}$. This case is symmetric to (b1).

It remains to consider case (c) when $\beta_{2} \leq \beta \leq \frac{\pi}{2}$. This case is exactly the same as case (a). This completes the proof of Theorem 2.2.

7.3. The proof of Theorem 2.3. Similar to the proof of Theorem 2.2, we employ (7.3) or (7.4) to prove Theorem 2.3. Since the convergence domain $\mathcal{D}$ is a rectangle for Category II, we have

$$
\eta^{(1)}=\eta^{(2)}=\tau \in \partial \Gamma
$$


and $\alpha_{c}=\min \left(\tau_{1} / c_{1}, \tau_{2} / c_{2}\right)$. We again consider three cases. Case (a) is for $0 \leq \beta<\beta_{1}$, case (b) is for $\beta=\beta_{1}$, and case (c) is for $\beta_{1}<\beta \leq \pi / 2$. As in the proof of Theorem 2.2, for some cases we are not able to establish the exact asymptotic for density $p_{c}(x)$. In these cases, we work with the moment generating function $\bar{\psi}_{c}(z)$ of the tail probability $q_{c}(x)$ directly, where $\bar{\psi}_{c}(z)$ is related to $\psi_{c}(z)$ through $(7.9)$.

(a) We assume that $0 \leq \beta<\beta_{1}$. In this case, $\alpha_{c}=\tau_{1} / c_{1}, \varphi_{2}\left(c_{1} z\right)$ is singular at $z=\alpha_{c}$ while $\varphi_{1}\left(c_{2} z\right)$ is analytic at $z=\alpha_{c}$. Furthermore, $\alpha_{c} c \neq$ $\theta^{(1, \mathrm{r})}$ and $z_{c}<\alpha_{c}$ because $\beta<\beta_{1}$. Therefore, $\zeta_{c}\left(\alpha_{c}\right) \neq 0$ and $\gamma_{2}(c) \neq 0$.

(a.i) Assume that $\tau_{1} \neq \theta_{1}^{(1, \max )}$, namely, $\eta^{(1)} \neq \theta^{(1, \max )}$. We first consider the case when $\eta^{(1)} \neq \theta^{(1, r)}$. By Lemma 6.6, $\varphi_{2}\left(c_{1} z\right)$ has a simple pole at $z=\alpha_{c}$ and $\varphi_{1}\left(c_{2} z\right)$ is analytic at $z=\alpha_{c}$. Using the exact same proof as the proof of (a.i) in Theorem 2.2, we prove that $q_{c}(x)$ has the exact asymptotic given by (7.8).

We next consider the case when $\eta^{(1)}=\theta^{(1, \mathrm{r})}$. In this case, by Lemma 6.6, $\varphi_{2}(z)$ has a double pole at $z=\tau_{1}$, thus $\varphi_{2}\left(c_{1} z\right)$ has a double pole at $z=\alpha_{c}$. Using the representation $(7.9)$, we see that $\bar{\psi}_{c}(z)$ has a double pole at $z=$ $\alpha_{c}$. One can check, just as in the proof of case (a.i) in Theorem 2.2, that conditions (C1a), (C1b) and (Cbc) are satisfied for $\bar{\psi}_{c}(z)$ with $m=1$ and $k_{0}=2$. By Lemma C.1, $q_{c}(x)$ has exact asymptotic that is given by (7.15).

(a.ii) Assume that $\tau_{1}=\theta_{1}^{(1, \max )}$, namely, $\eta^{(1)}=\theta^{(1, \max )}$. The proof is similar to the proof of (a.ii) in Theorem 2.2. When $\theta^{(1, \mathrm{r})}=\theta^{(1, \max )}, p_{c}(x)$ has exact asymptotic that is given by (7.8), where we have critically used (6.27) instead of $(6.24)$. When $\theta^{(1, \mathrm{r})} \neq \theta^{(1, \max )}, p_{c}(x)$ has exact asymptotic that is given by (7.14), where we have critically used (6.28) instead of (6.25).

(b) We assume $\beta=\beta_{1}$. In this case, we always have $\zeta_{c}\left(\alpha_{c}\right)=0$. We first consider the case $\tau=\theta^{(1, \mathrm{r})}$. In this case, $\alpha_{c} c=\tau=\theta^{(1, \mathrm{r})}$, which implies $\gamma_{2}(c)=0$. Thus, $\psi_{c}(z)$ has representation (7.7). Because $\zeta_{c}\left(\alpha_{c}\right)=0$, $\gamma_{1}(c)>0$, and it follows from (7.7), (7.5), and (7.16) that for some $\epsilon>0$

$$
\bar{\psi}_{c}(z)=-\frac{\gamma_{1}(c)}{\langle c, \Sigma c\rangle} \frac{\varphi_{1}\left(c_{2} z\right)}{z\left(\alpha_{c}-z\right)}-\frac{1}{z} \quad \text { for } 0<\Re z<\alpha_{c}+2 \epsilon
$$

except at $z=\alpha_{c}$. By Lemma $6.7, \varphi_{1}\left(c_{2} z\right)$ has a simple pole at $z=\alpha_{c}$. Thus, $\bar{\psi}_{c}(z)$ has a double pole at $z=\alpha_{c}$. Hence, $\bar{\psi}_{c}(z)$ satisfies condition (C1a) of Lemma C.1 with $m=1$ and $k_{0}=2$. Similar to the proof for case (a.i) of Theorem 2.2, we can prove that conditions (C1b) and (C1c) of Lemma C.1 are satisfied for $\bar{\psi}_{c}(z)$. Therefore, Lemma C.1 implies that $q_{c}(x)$ has the exact asymptotic given by (7.15).

We next consider the case $\tau \neq \theta^{(1, \mathrm{r})}$ for $\beta=\beta_{1}$. In this case $\zeta_{c}\left(\alpha_{c}\right)=0$, $\gamma_{2}(c)>0$, and $\gamma_{1}(c)>0$. When $\tau_{1}<\theta_{1}^{(1, \max )}$, both $\varphi_{1}\left(c_{2} z\right)$ and $\varphi_{2}\left(c_{1} z\right)$ 
have a simple pole at $z=\alpha_{c}$, and $\bar{\psi}_{c}(z)$ has representation (7.9). Because $\zeta_{c}\left(\alpha_{c}\right)=0$, it follows from $(7.9)$ that $\bar{\psi}_{c}(z)$ has a double pole at $z=\alpha_{c}$. Hence, $\bar{\psi}_{c}(z)$ satisfies condition (C1a) of Lemma C.1 with $m=1$ and $k_{0}=2$. Following the proof as in case (a.i) of Theorem 2.2, conditions (C1b) and (C1c) of Lemma C.1 are satisfied for $\bar{\psi}_{c}(z)$. Thus, Lemma C.1 implies that $q_{c}(x)$ has the exact asymptotic given by $(7.15)$. When $\tau_{1}=\theta_{1}^{(1, \max )}, \varphi_{1}\left(c_{2} z\right)$ has a simple pole at $z=\alpha_{c}$, and $\varphi_{2}\left(c_{1} z\right)$ is analytic in $\mathcal{G}_{\delta}\left(\alpha_{c}\right)$, but is singular at $z=\alpha_{c}$. By Lemma D.2,

$$
\frac{1}{\zeta_{c}(z)} \varphi_{1}\left(c_{2} z\right) \quad \text { and } \quad \frac{1}{\zeta_{c}(z)} \varphi_{2}\left(c_{1} z\right)
$$

are moment generating functions for some finite measures $\xi_{1}$ and $\xi_{2}$, respectively. Similar to (7.5), there exists an $\epsilon>0$ such that the moment generating function for $\xi_{1}([x, \infty))$ is given by

$\bar{\psi}_{1}(z)=\frac{1}{z}\left(\frac{1}{\zeta_{c}(z)} \varphi_{1}\left(c_{2} z\right)-\zeta_{c}(0) \varphi_{1}(0)\right)=-\frac{\gamma_{1}(c)}{\langle c, \Sigma c\rangle} \frac{\varphi_{1}\left(c_{2} z\right)}{z\left(\alpha_{c}-z\right)}-\zeta_{c}(0) \varphi_{1}(0) \frac{1}{z}$,

for $\Re z<\alpha_{c}+2 \epsilon$ except at $z=\alpha_{c}$. Because Category II implies that $\tau_{2}<$ $\theta_{2}^{(2, \max )}$, by Lemma 6.7 , one can check that $\bar{\psi}_{1}(z)$ satisfies condition (C1a) of Lemma C. 1 with $m=1$ and $k_{0}=2$. Following the same proof as in case (a.i) of Theorem 2.2 , we can prove that $\bar{\psi}_{1}(z)$ satisfies conditions ( C1b) and (C1c) of Lemma C.1. Thus, by Lemma C.1, $\xi_{1}([x, \infty))$ has exact asymptotic $x e^{-\alpha_{c} x}$ as $x \rightarrow \infty$. For the second moment generating function, it is analytic in $\mathcal{G}_{\delta}\left(\alpha_{c}\right)$, thus it satisfies condition (C2a) of Lemma C.2. Lemma 6.8 implies that

$$
\lim _{\substack{z \rightarrow \alpha_{c} \\ z \in \mathcal{G}_{\delta}\left(\alpha_{c}\right)}}\left(\alpha_{c}-z\right)^{1 / 2} \frac{1}{\zeta_{c}(z)} \varphi_{2}\left(c_{1} z\right)
$$

exists and is nonzero by (7.13). Thus, the moment generating function satisfies condition (C2c) of Lemma C.2. Also, inequality (6.11) implies condition (C2b) of Lemma C.2. Thus, we can apply Lemma C.2 to conclude that $\xi_{2}([x, \infty))$ has exact asymptotic $x^{-1 / 2} e^{-\alpha_{c} x}$. Because $\gamma_{1}(c)>0, \gamma_{2}(c)>0$ and $z_{c}=\alpha_{c}$, by (7.3) and Lemma D.1, the exact asymptotic of $q_{c}(x)$ is given by $(7.15)$.

(c) We assume $\beta_{1}<\beta \leq \pi / 2$. In this case, $c_{2} \alpha_{c}=\tau_{2}$ and $\gamma_{1}\left(\theta^{(2, \mathrm{r})}\right)=0$. Because $\alpha_{c}<\tau_{1} / c_{1}, \varphi_{2}\left(c_{1} z\right)$ is analytic at $z=\alpha_{c}$. If $\beta=\beta_{2}^{(\mathrm{r})}$, then $\gamma_{1}(c)=0$ but $\gamma_{2}(c)>0$. Thus, (7.3) reduces to

$$
\psi_{c}(z)=\frac{\gamma_{2}(c)}{\zeta_{c}(z)} \varphi_{2}\left(c_{1} z\right)
$$


Since $\zeta_{c}\left(\alpha_{c}\right)=0$, we can apply Lemma D.4, and therefore $p_{c}(x)$ has the exact asymptotic given by (7.8). If $\beta \neq \beta_{2}^{(\mathrm{r})}$, then $\zeta_{c}\left(\alpha_{c}\right) \neq 0$. We here can not have $\gamma_{1}(c)=0$ because the latter would imply (7.17), contradicting the fact that $\psi_{c}(z)$ is singular at $z=\alpha_{c}$. Therefore, we have $\zeta_{c}\left(\alpha_{c}\right) \neq 0$ and $\gamma_{1}(c) \neq 0$. Therefore, $\bar{\psi}_{c}(z)$ has representation (7.9), from which we conclude that $\bar{\psi}_{c}(z)$ has a simple pole at $z=\alpha_{c}$. Following the same proof as in (a.i) of Theorem 2.2, we conclude that $q_{c}(x)$ has exact asymptotic $f_{c}(x)$ that is given by (7.8). This completes the proof of Theorem 2.3.

8. Concluding remarks. In this section, we briefly discuss four topics. They are the non-singular assumption on the covariance matrix $\Sigma$, the large deviations rate function, the fine exact asymptotics, and an extension to SRBMs in more than two dimensions.

Singular covariance matrix. Throughout this paper, we have assumed that $\Sigma$ is positive definite, which is equivalent to be non-singular. In applications, the covariance matrix $\Sigma$ may be singular. Consider, for example, a tandem queue that has a single Poisson arrival process at station 1. Each station has a single server, and the service times at each station are deterministic. In this case, the two-dimensional queue length process can be modeled by an SRBM that has a singular covariance matrix [12]. When $\Sigma$ is singular, the ellipse $\partial \Gamma$ becomes a parabolic curve and the set $\Gamma$ is not bounded. The exact asymptotics analysis should be analogous to the analysis in this paper, but the detailed analysis is not attempted in this paper.

Large deviations. We next consider the connection of convergence domain $\mathcal{D}$ with large deviations. As we discussed in Section 1, the rough asymptotics of $\mathbb{P}(Z(\infty) \in u B)$ may be interesting for $B \in \mathcal{B}\left(\mathbb{R}_{+}^{2}\right)$. We here consider the large deviations rate function $I$ in (1.11) and (1.12). Let $B$ be any convex, closed set of $\mathbb{R}_{+}^{2}$ and $u$ be a positive number. Since $Z(\infty) \in u B$ implies

$$
u \inf _{v \in B}\langle v, \theta\rangle \leq\langle\theta, Z(\infty)\rangle \text { for each } \theta \in \mathbb{R}^{2},
$$

we have

$$
e^{u \inf _{v \in B}\langle v, \theta\rangle} \mathbb{P}(Z(\infty) \in u B) \leq \mathbb{E}\left(e^{\langle\theta, Z(\infty)\rangle}\right)=\varphi(\theta),
$$

where $\inf _{v \in B}\langle v, \theta\rangle$ may be $-\infty$, for which the above inequality is trivial. Hence, as long as $\varphi(\theta)<\infty$, that is, $\theta \in \mathcal{D}$,

$$
\limsup _{u \rightarrow \infty} \frac{1}{u} \log \mathbb{P}(Z(\infty) \in u B) \leq-\inf _{v \in B}\langle v, \theta\rangle,
$$

and therefore, letting $\mathcal{D}_{\min }=\left\{\theta \in \mathcal{D} ; \theta_{1} \geq \theta_{1}^{(1, \min )}, \theta_{2} \geq \theta_{2}^{(2, \min )}\right\}$, we have

$$
\limsup _{u \rightarrow \infty} \frac{1}{u} \log \mathbb{P}(Z \in u B) \leq-\sup _{\theta \in \mathcal{D}} \inf _{v \in B}\langle v, \theta\rangle \leq-\sup _{\theta \in \mathcal{D}_{\min }} \inf _{v \in B}\langle v, \theta\rangle .
$$




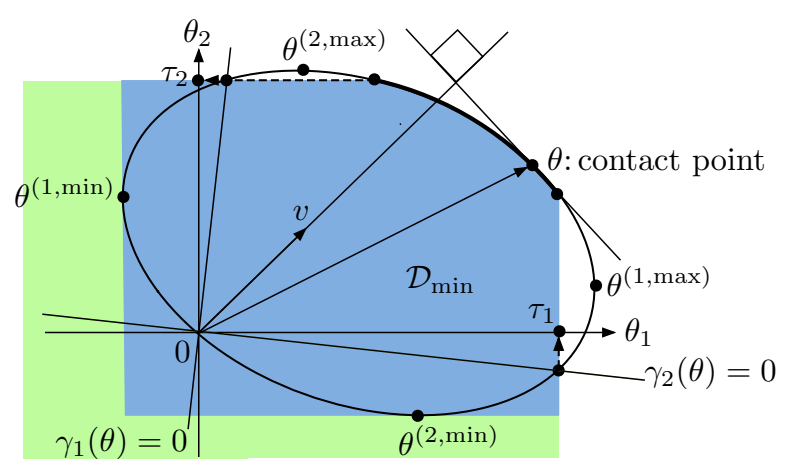

FIG 13. The supremum of $\langle v, \theta\rangle$ for $\theta \in \mathcal{D}$ attains on $\overline{\mathcal{D}}_{\text {min }}$

Since $\mathcal{D}_{\text {min }}$ is a bounded convex set and $\langle v, \theta\rangle$ is a continuous concaveconvex function when either $\theta$ or $v$ is fixed, by Corollary 37.3.2 of [28], we have

$$
\sup _{\theta \in \mathcal{D}_{\text {min }}} \inf _{v \in B}\langle v, \theta\rangle=\inf _{v \in B} \sup _{\theta \in \mathcal{D}_{\text {min }}}\langle v, \theta\rangle
$$

Furthermore, from the fact that $\langle\theta, v\rangle$ for $\theta \in \mathcal{D}$ attains the supremum on the closure of $\mathcal{D}_{\text {min }}$ for any $v \in \mathbb{R}_{+}^{2}$ (see Figure 13), the right side of the above inequality equals $\inf _{v \in B} \sup _{\theta \in \mathcal{D}}\langle v, \theta\rangle$. Hence, (8.1) yields

$$
\limsup _{u \rightarrow \infty} \frac{1}{u} \log \mathbb{P}(Z \in u B) \leq-\inf _{v \in B} \sup _{\theta \in \mathcal{D}}\langle v, \theta\rangle
$$

Thus, if the rate function $I$ exists, then, from (1.12), we have

$$
I(v) \geq J(v) \equiv \sup _{\theta \in \mathcal{D}}\langle v, \theta\rangle
$$

Furthermore, comparing (8.2) with the rate function $I$ in (1.11), we can say this $J$ is a very good candidate for the rate function $I$. One may ask whether $J$ is indeed the rate function using the $I$ obtained in $[1,11]$. This question is also related to how the optimal path in the sample path large deviations is related to the present results. These questions will be answered in a subsequent paper. In particular, it will be shown that the equality in (8.3) holds if and only if $\tau \notin \mathcal{D}$.

Another interesting question related to the large deviations is the exact asymptotics of $\mathbb{P}(Z(\infty) \in x v+B)$ for each directional vector $v \geq 0$ and a closed subset $B$ of $\mathbb{R}_{+}^{2}$. This is a harder problem. It will also be investigated in the subsequent paper. 
Fine exact asymptotics. For each direction $c \in \mathbb{R}_{+}^{2}$, we have derived the exact tail asymptotics for tail probability $q_{c}(x)$ in the form $x^{\kappa} e^{-\alpha x}$ through the left-most singular point of $\psi_{c}$. If we carefully examine our proofs in Section 7.2, we can find its second left-most singular point in many cases. This suggests a possibility to obtain a finer asymptotic function than the one given in (1.6). That is, we may refine the tail asymptotics in the form of (1.10). However, it is notable that constants $b_{c}, b_{d}$ there can not be obtained in general. Here, we present fine asymptotics $f_{c}(x)$ for $q_{c}(x)$ as $x \rightarrow \infty$ for three cases. They illustrate the potential and difficulty for pursuing general cases.

We assume $\tau \notin \bar{\Gamma}$. Thus, we are always in Category I. Furthermore, $\eta^{(1)}$ can not be identical with $\eta^{(2)}$, and line $\tau$ is strictly above $\eta^{(1)}$ and strictly below $\eta^{(2)}$. We further assume that $\tau_{1}<\theta_{1}^{(1, \max )}$ and $\tau_{2}<\theta_{2}^{(2, \max )}$ (see Figure 3).

(i) $c$ is strictly above $\eta^{(1)}$ and below or on line $\tau$ : (see case (b) of Theorem 2.2):

We first assume that $c$ is strictly below line $\tau$. The left-most singular point $\alpha_{c}$ of $\psi_{c}(z)$ is the root of $\zeta_{c}(z)$ by (7.3). Hence, $\psi_{c}(z)$ has a simple pole at $z=z_{c}$. The next singularity of $\psi_{c}(z)$ occurs when either $\varphi_{1}\left(c_{2} z\right)$ or $\varphi_{2}\left(c_{1} z\right)$ is singular at $z$. Because $c$ is below line $\tau$, we have $\frac{\tau_{1}}{c_{1}}<\frac{\tau_{2}}{c_{2}}$. Then, $\varphi_{2}\left(c_{1} z\right)$ is singular at point $z=\frac{\tau_{1}}{c_{1}}$, which is smaller than $\frac{\tau_{2}}{c_{2}}$, the singular point of $\varphi_{1}\left(c_{2} z\right)$. Therefore, $z=\frac{\tau_{1}}{c_{1}}$ is the second left-most singular point of $\psi_{c}(z)$. We decompose $\psi_{c}$ of $(7.3)$ as

$$
\psi_{c}(z)=g_{0}(z)+g_{1}(z)
$$

where

$$
\begin{aligned}
& g_{0}(z)=\frac{1}{\zeta_{c}(z)}\left(\gamma_{2}(c) \varphi_{2}\left(c_{1} \alpha_{c}\right)+\gamma_{1}(c) \varphi_{1}\left(c_{2} z\right)\right), \\
& g_{1}(z)=\frac{\gamma_{2}(c)}{\zeta_{c}(z)}\left(\varphi_{2}\left(c_{1} z\right)-\varphi_{2}\left(c_{1} \alpha_{c}\right)\right) .
\end{aligned}
$$

It is easy to see that $g_{0}(z)$ has a simple pole at $z=\alpha_{c}$, and is analytic for $\Re z<\frac{\tau_{2}}{c_{2}}$ except for this pole. On the other hand, $g_{1}(z)$ has a removable pole at $z=\alpha_{c}$, and is analytically extendable for $\Re z<\frac{\tau_{1}}{c_{1}}$, and singular at $z=\frac{\tau_{1}}{c_{1}}$. In this case, $\varphi_{2}\left(c_{1} z\right)$ has a simple pole there by Lemma 6.6 because of the assumption $\tau_{1}<\theta_{1}^{(1, \max )}$. Hence, by applying Lemma D.4 for $g_{0}$ and Lemma C. 1 for $g_{1}$, we have

$$
f_{c}(x)=b_{c} e^{-z_{c} x}+b_{d} e^{-\frac{\tau_{1}}{c_{1}} x} .
$$

where $b_{c}$ and $b_{d}$ are positive constants. 
(ii) $c$ is on line $\tau$ :

In this case, $\frac{\tau_{1}}{c_{1}}=\frac{\tau_{2}}{c_{2}}$. Hence, both of $\varphi_{1}\left(c_{2} z\right)$ and $\varphi_{2}\left(c_{1} z\right)$ have singularity at $z=\frac{\tau_{1}}{c_{1}}=\frac{\tau_{2}}{c_{2}}$. We then can use a similar argument as in (i) to arrive at the same fine asymptotic (8.4).

(iii) $c$ is on line $\eta^{(1)}$ :

In this case, $z_{c}=\alpha_{c}=\tau_{1} / c_{1}$ and $\varphi_{2}(z)$ has a simple pole at $z=\tau_{1}$. Hence, the leftmost singularity of $\psi_{c}(z)$ is a double pole at $z=\alpha_{c}$, and we need to find the singular point which has a smallest real part from $\varphi_{1}\left(c_{2} z\right)$ and $\varphi_{2}\left(c_{1} z\right)$ for $\Re z>\alpha_{c}$. However, finding the second singularity of $\varphi_{2}\left(c_{1} z\right)$ is a bit complicated. To determine its second singularity, we use (6.8). Because $\tau_{2}>c_{2}>\theta_{2}^{(1, \max )}$, following the proof of Lemma 6.6, except at $z=\tau_{1}, \varphi_{2}(z)$ has an analytic extension for $\Re z<\theta_{1}^{(1, \max )}$. Therefore, except at $z=\tau_{1} / c_{1}, \varphi_{2}\left(c_{1} z\right)$ has an analytic extension for $\Re z<\theta_{1}^{(1, \max )} / c_{1}$. If $\tau_{2} / c_{2}<\theta_{1}^{(1, \max )} / c_{1}$, then $\tau_{2} / c_{2}$ is the second left-most singularity of $\psi_{c}(z)$. By applying Lemma C.1, the tail distribution $q_{c}(x)$ has the exact asymptotic:

$$
f_{c}(x)=b_{c} x e^{-z_{c} x}+b_{d} e^{-\frac{\tau_{2}}{c_{2}} x} .
$$

Because $\underline{f}_{2}(z)$ has a branch point at $z=\theta_{1}^{(1, \max )}, \varphi_{2}\left(c_{1} z\right)$ should be singular at $z=\theta_{1}^{(1, \max )} / c_{1}$. However, we are not able to verify this claim. Therefore, when $\tau_{2} / c_{2} \geq \theta_{1}^{(1, \max )} / c_{1}$, we are not able to obtain a fine asymptotic.

High dimensional SRBMs. The results in this paper may be extended to cover SRBMs in $d$ dimensions, where $d \geq 3$. Indeed, Miyazawa and Kobayashi [25] have conjectured such extensions. We hope the technical results in the present paper will be useful to prove these challenging conjectures.

\section{APPENDIX A: DEFINITION OF AN SRBM}

In this section, we present the standard definition of a semimartingale reflecting Brownian motion (SRBM) in the $d$-dimensional orthant $\mathbb{R}_{+}^{d}$, where $d$ is a positive integer. We also review the standard definition of positive recurrence for an SRBM, connecting it with the alternative definition used in Section 1.

Recall from Section 1 that $\mu$ is a constant vector in $\mathbb{R}^{d}, \Sigma$ is a $d \times d$ symmetric and strictly positive definite matrix, and $R$ is a $d \times d$ matrix. We shall define an SRBM associated with the data $\left(\mathbb{R}_{+}^{d}, \mu, \Gamma, R\right)$. For this, a triple $\left(\Omega, \mathcal{F},\left\{\mathcal{F}_{t}\right\}\right)$ will be called a filtered space if $\Omega$ is a set, $\mathcal{F}$ is a $\sigma$-field of 
subsets of $\Omega$, and $\left\{\mathcal{F}_{t}\right\} \equiv\left\{\mathcal{F}_{t}, t \geq 0\right\}$ is an increasing family of sub- $\sigma$-fields of $\mathcal{F}$, i.e., a filtration.

Definition A.1. An SRBM associated with $\left(\mathbb{R}_{+}^{d}, \mu, \Gamma, R\right)$ is a continuous $\left\{\mathcal{F}_{t}\right\}$-adapted $d$-dimensional process $Z=\{Z(t), t \geq 0\}$, together with a family of probability measures $\left\{\mathbb{P}_{x}, x \in S\right\}$, defined on some filtered space $\left(\Omega, \mathcal{F},\left\{\mathcal{F}_{t}\right\}\right)$ such that, for each $x \in S$, under $\mathbb{P}_{x},(1.1)$ and (1.4) hold, where, writing $W(t)=X(t)-\mu t$ for $t \geq 0, W$ is a $d$-dimensional Brownian motion with covariance matrix $\Sigma$, an $\left\{\mathcal{F}_{t}\right\}$-martingale such that $W(0)=x \mathbb{P}_{x}$-a.s., and $Y$ is an $\left\{\mathcal{F}_{t}\right\}$-adapted $d$-dimensional process such that $\mathbb{P}_{x}$-a.s. $(1.2)$ and (1.3) hold. Here (1.2) is interpreted to hold for each component of $Y$, and (1.3) is defined to be

$$
\int_{0}^{t} 1_{\left\{Z_{i}(s) \neq 0\right\}} d Y_{i}(s)=0 \quad \text { for all } t \geq 0 .
$$

Definition A.1 gives the so-called weak formulation of an SRBM. It is a standard definition adopted in the literature; see, for example, [7] and [30]. Note that condition (A.1) is equivalent to the condition that, for each $t>0$, $Z_{j}(t)>0$ implies $Y_{j}(t-\delta)=Y_{j}(t+\delta)$ for some $\delta>0$. [27] showed that a necessary condition for a $\left(\mathbb{R}_{+}^{d}, \mu, \Sigma, R\right)$-SRBM to exist is that the reflection matrix $R$ is completely- $\mathcal{S}$ (this term was defined in Section 1). [29] showed that when $R$ is completely- $\mathcal{S}$, a $\left(\mathbb{R}_{+}^{d}, \mu, \Sigma, R\right)$-SRBM $Z$ exists and $Z$ is unique in law under $\mathbb{P}_{x}$ for each $x \in S$. Furthermore, $Z$, together with the family of probability measures $\left\{\mathbb{P}_{x}, x \in \mathbb{R}_{+}^{d}\right\}$, is a Feller continuous strong Markov process.

\section{APPENDIX B: IMPOSSIBLE CATEGORY}

We show that the other category than Categories I, II and III is impossible.

Lemma B.1. The following category cannot occur:

$$
\theta_{1}^{(2, \Gamma)}>\theta_{1}^{(1, \Gamma)} \text { and } \theta_{2}^{(1, \Gamma)}>\theta_{2}^{(2, \Gamma)}
$$

Proof. Assume that (B.1) holds. Since $\gamma_{2}\left(\theta^{(1, \Gamma)}\right) \leq 0$ and $\gamma_{1}\left(\theta^{(2, \Gamma)}\right) \leq 0$, we have

$$
\theta_{1}^{(2, \Gamma)} r_{11}+\theta_{2}^{(2, \Gamma)} r_{21} \leq 0, \quad \theta_{1}^{(1, \Gamma)} r_{12}+\theta_{2}^{(1, \Gamma)} r_{22} \leq 0 .
$$

Since $r_{11}, r_{22}>0$, then (B.1) implies that

$$
\theta_{1}^{(1, \Gamma)} r_{11}+\theta_{2}^{(2, \Gamma)} r_{21}<0, \quad \theta_{1}^{(1, \Gamma)} r_{12}+\theta_{2}^{(2, \Gamma)} r_{22}<0 .
$$


These inequalities imply $r_{21}, r_{12}<0$ since $\theta_{k}^{(k, \Gamma)} \geq 0$. It follows that

$$
\theta_{1}^{(1, \Gamma)}\left(r_{11} r_{22}-r_{12} r_{21}\right)<0 \text {. }
$$

This is impossible because of $\theta_{1}^{(1, \Gamma)} \geq 0$ and the stability condition (2.2).

\section{APPENDIX C: COMPLEX INVERSION TECHNIQUE}

As we planed in Section 6, we use complex variable moment generating functions for proving Theorems 2.2 and 2.3. In this section, we first present classical results for obtaining exact tail asymptotics from a complex variable moment generating function. For this, we refer to Doetsch [5] similarly to [26].

Let $f$ be a nonnegative valued, continuous and integrable function on $[0, \infty)$, and define the complex variable function $g$ as

$$
g(z)=\int_{0}^{\infty} e^{z x} f(x) d x, \quad \Re z<\alpha_{0},
$$

where $\alpha_{0}=c_{p}(g)(\equiv \sup \{\theta \geq 0: g(\theta)<\infty\})$. By Lemma 6.1, $\alpha_{0}$ is the leftmost singular point of $g(z)$, and $g(z)$ is analytic for $\Re z<\alpha_{0}$. We analytically expand this function $g$, which is also denoted by $g$. We are interested in the following two cases for $\alpha_{0}>0$.

(C1) $g(z)$ has a pole at $z=\alpha_{0}$, and there is a positive number $\alpha_{1}>\alpha_{0}$ such that $g(z)$ is analytic except for this pole for $\Re z<\alpha_{1}$.

(C2) $g(z)$ has a branch point at $z=\alpha_{0}$, and there is an angle $\delta \in\left[0, \frac{1}{2} \pi\right)$ such that $g(z)$ is analytic on the set $\mathcal{G}_{\delta}\left(\alpha_{0}\right) \equiv\left\{z \in \mathbb{C}: z \neq \alpha_{0}, \mid \arg (z-\right.$ $\left.\alpha_{0}\right) \mid>\delta$, where $\arg z \in(-\pi, \pi)$ is the principal part of the argument of complex number $z$.

The basic idea for case (C1) is extracting a suitable function $g_{0}(z)$ from $g(z)$ so that $g(z)-g_{0}(z)$ is analytic $\Re z<\alpha_{1}$. From the assumptions in (C1), $g_{0}(z)$ has a pole at $z=\alpha_{0}$. Let $k \geq 1$ be the order of this pole. Then,

$$
g_{0}(z)=\frac{c_{0}}{\left(\alpha_{0}-z\right)^{k}}
$$

for some constant $c_{0}>0$. Choose $\bar{\alpha}$ such that $\alpha_{0}<\bar{\alpha}<\alpha_{1}$. Let $L_{v}$ be the boundary of the rectangle $\{z \in \mathbb{C} ; 0 \leq \Re z \leq \bar{\alpha},|\Im z| \leq v\}$ for each $v>0$. Then, from the Cauchy integral formula, we have

$$
\frac{1}{2 \pi i} \int_{L_{v}} g_{0}(z) e^{-z x} d z=\frac{c_{0}}{\Gamma(k)} x^{k-1} e^{-\alpha_{0} x}, \quad x \geq 0 .
$$


Denote the right side function by $f_{0}(t)$, and decompose $g$ as

$$
g(z)=g_{0}(z)+g(z)-g_{0}(z)
$$

Because $g(z)-g_{0}(z)$ is analytic on the rectangle,

$$
\int_{\partial L_{v}} e^{-x z}\left(g(z)-g_{0}(z)\right) d z=0
$$

Under the assumption (C1): $f$ is nonnegative, $\int_{0}^{\infty} f(x) d x<\infty$ and $f(x)$ is continuous in $(0, \infty)$, the inversion formula

$$
f(x)=\frac{1}{2 \pi i} \int_{-i \infty}^{+i \infty} e^{-x z} g(z) d z
$$

always holds. Assume that

$$
\lim _{|y| \rightarrow \infty} \sup _{x \in\left[\alpha_{0}, \alpha_{1}\right]}|g(x+i y)|=0
$$

and the integral $\int_{-\infty}^{+\infty} e^{-i x y} g(\bar{\alpha}+i y) d y$ converges at a fixed $x>0$. Applying the counter integral on $L_{v}$ to both sides of equation (C.2) and letting $v \rightarrow \infty$, we have for $x>0$

$$
\begin{aligned}
f(x) & =\frac{1}{2 \pi i} \int_{-i \infty}^{+i \infty} e^{-x z} g(z) d z=f_{0}(x)+\frac{1}{2 \pi i} \int_{\bar{\alpha}-i \infty}^{\bar{\alpha}+i \infty} e^{-x z} g(z) d z \\
& =f_{0}(x)+\frac{1}{2 \pi i} e^{-\bar{\alpha} x} \int_{-\infty}^{+\infty} e^{-i x y} g(\bar{\alpha}+i y) d y
\end{aligned}
$$

If the integral $\int_{-\infty}^{+\infty} e^{-i x y} g(\bar{\alpha}+i y) d y$ uniformly converges for $x>T$, where $T>0$ is some constant, by the Riemann-Lebesgue Lemma (c.f. [5, Theorem 23.3]),

$$
\lim _{x \rightarrow \infty} \int_{-\infty}^{+\infty} e^{-i x y} g(\bar{\alpha}+i y) d y=0 .
$$

Because $\bar{\alpha}>\alpha_{0}$, we have $f(x)=f_{0}(x)+o\left(e^{-\bar{\alpha} x}\right)$ as $x \rightarrow \infty$.

We can similarly prove the following lemma, which is a special case of Theorem 35.1 in [5].

Lemma C.1. Let $g$ be the moment generating function of a nonnegative, continuous and integrable function $f$. If the following conditions are satisfied for some integer $m \geq 1$ and some number $\alpha_{m}>0$, 
(C1a) there is a complex variable function $g_{0}(z)$ such that, some integers $k_{j}$ and some numbers $c_{j}, \alpha_{j}$ for $j=0,1, \ldots m-1$ such that $0<\alpha_{0}<\alpha_{1}<\cdots<\alpha_{m-1}<\alpha_{m}$ and

$$
g_{0}(z)=\sum_{j=0}^{m-1} \frac{c_{j}}{\left(\alpha_{j}-z\right)^{k_{j}}}
$$

and $g(z)-g_{0}(z)$ is analytic for $\Re z<\alpha_{m}$,

(C1b) $g(z)$ uniformly converges to 0 as $z \rightarrow \infty$ for $0 \leq \Re z \leq \alpha_{m}$,

(C1c) the integral

$$
\int_{-\infty}^{+\infty} e^{-i y x} g\left(\alpha_{m}+i y\right) d y
$$

uniformly converges for $x>T$ for some $T>0$,

then

$$
f(x)=\sum_{j=0}^{m-1} \frac{c_{j}}{\Gamma\left(k_{j}\right)} x^{k_{j}-1} e^{-\alpha_{j} x}+o\left(e^{-\alpha_{m} x}\right), \quad x \rightarrow \infty,
$$

where conditions (C1b) and (C1c) are satisfied if, for some constants $a, b, \delta>0$,

$$
|g(z)|<\frac{a}{|z|^{1+\delta}}, \quad \Re z \in\left[0, \alpha_{m}\right],|\Im z|>b .
$$

For case $(\mathrm{C} 2)$, the situation is a bit complicated, but the idea is essentially the same. We need the counter integral along the boundary of $\mathcal{G}_{\delta}\left(\alpha_{0}\right)$ instead of Fourier inversion formula. The following lemma is a special case of Theorem 37.1 of Doetsch [5].

Lemma C.2. For the $f, g$ of Lemma C.1, if the following two conditions hold for some $\alpha>0$ and some $\delta \in\left[0, \frac{\pi}{2}\right)$ :

(C2a) $g(z)$ is analytic on $\mathcal{G}_{\delta}(\alpha)$,

(C2b) $g(z) \rightarrow 0$ as $|z| \rightarrow \infty$ for $z \in \mathcal{G}_{\delta}(\alpha)$,

(C2c) for some $a \in \mathbb{R}, \lambda \in \mathbb{R}$ and $c_{0} \in \mathbb{R}$,

$$
\lim _{\substack{z \rightarrow \alpha \\ z \in \mathcal{G}_{\delta}(\alpha)}}(\alpha-z)^{\lambda}(g(z)-a)=c_{0}
$$

then

$$
f(x)=\frac{c_{0}}{\Gamma(\lambda)} x^{\lambda-1} e^{-\alpha x}(1+o(1))
$$


where, $\Gamma(\lambda)$ is the Gamma function as defined in Section 53 of Volume II of [23] (see also Theorem 10.14 in its Section 54 for its integral representation), and by convention $\frac{1}{\Gamma(\lambda)}=0$ when $\lambda=0,-1,-2, \ldots$.

\section{APPENDIX D: DECOMPOSING A MOMENT GENERATING FUNCTION}

In application of Lemmas C.1 and C.2, we need to verify that the density function $f$ is continuous in $(0, \infty)$. Furthermore, we may need to decompose the moment generating function $g$ of interest into the linear combination of moment generating functions for which those lemmas are applicable. We present their details in this appendix. For convenience, we refer to an integrable nonnegative function as a density. We first note the following basic fact.

Lemma D.1. Let $g_{1}$ and $g_{2}$ be the moment generating functions of finite measures $\xi_{1}$ and $\xi_{2}$ on $[0, \infty)$. For any non zero real constants $d_{1}$ and $d_{2}$, let

$$
g(s)=d_{1} g_{1}(s)+d_{2} g_{2}(s) \quad \text { for } s \leq 0 .
$$

Then $g$ is the moment generating function of the signed measure $\xi$ defined by

$$
\xi(B)=d_{1} \xi_{1}(B)+d_{2} \xi_{2}(B), \quad B \in \mathcal{B}([0, \infty)) .
$$

In particular, if $\xi_{1}$ and $\xi_{2}$ have densities $f_{1}$ and $f_{2}$, then $\xi$ has density $f$

$$
f(x)=d_{1} f_{1}(x)+d_{2} f_{2}(x) \quad \text { for } x \geq 0 .
$$

In verifying the continuity of densities as well as decomposing moment generating functions, the following two results are useful.

Lemma D.2. Assume that $g$ is the moment generating function of a finite measure $\xi$ on $[0, \infty)$. Let $s_{0}>0$ be fixed. Define, for $k \in\{1,2, \ldots\}$,

$$
h(s)=\frac{g(s)}{\left(s_{0}-s\right)^{k}} \quad \text { for } s \leq 0 .
$$

Then $h$ is the moment generating function of some density function on $[0, \infty)$. In particular, this density is continuous if $\xi$ has has no atom.

Proof. Let $g_{1}(s)=\left(s_{0} /\left(s_{0}-s\right)\right)^{k}$ for $s \leq 0$. Then $g_{1}(s)$ is the moment generating function of the Erlang distribution with order $k$ and mean $\left(k s_{0}\right)^{-1}$. Clearly, $h(s)=g_{1}(s) g_{2}(s)$ for $s \leq 0$, where $g_{2}(s)=g(s) / s_{0}^{k}$ is the 
moment generating function of measure $\xi / s_{0}^{k}$. Therefore, $h$ is the moment generating function of the convolution of measure $\xi / s_{0}^{k}$ and the Erlang distribution. Since the Erlang distribution has a density, this convolution must have a density. The remaining statement is immediate from this convolution and the fact that $\xi$ has no atom.

Lemma D.3. Let $g$ be the same as in Lemma D.2, and assume $g\left(s_{1}\right)$ is finite for some constant $s_{1} \in \mathbb{R}$. Let

$$
h(s)=\frac{g\left(s_{1}\right)-g(s)}{s_{1}-s}, \quad s<s_{1} .
$$

Then $h$ is also the moment generating function of a density function on $[0, \infty)$, and has the same convergence parameter as $g$ (see Lemma 6.1 for its definition). In particular, this density is continuous if $\xi$ has no atom.

Proof. The statement on the convergence parameter is immediate from the definition of $h$. For $s<s_{1}$,

$$
\begin{aligned}
h(s) & =\int_{0}^{\infty} \frac{e^{\left(s-s_{1}\right) u}-1}{s-s_{1}} e^{s_{1} u} \xi(d u) \\
& =\int_{0}^{\infty} \int_{0}^{u} e^{\left(s-s_{1}\right) x} d u e^{s_{1} u} \xi(d u) \\
& =\int_{0}^{\infty} \int_{x}^{\infty} e^{s_{1}(u-x)} \xi(d u) e^{s x} d x .
\end{aligned}
$$

Hence, $h(s)$ is the moment generating function of the density $e^{-s_{1} x} \int_{x}^{\infty} e^{s_{1} u} \times$ $\xi(d u)$, which is continuous in $x$ if $\xi$ has no atom.

In application of Lemma C.1, the condition (C1b) is annoying. In our application, the following result is sufficient.

Lemma D.4. Assume a complex variable function $h$ is given by (D.2) with $g$ such that $\xi$ has no atom. Denote a continuous density on $[0, \infty)$ whose moment generating function is $h$ by $\tilde{f}$. If $g\left(s_{0}+\epsilon\right)<\infty$ for some $\epsilon>0$, then

$$
\tilde{f}(x)=\frac{g\left(s_{0}\right)}{(k-1) !} x^{k-1} e^{-s_{0} x}+o\left(x^{k-1} e^{-s_{0} x}\right), \quad x \rightarrow \infty .
$$

Proof. For $k=1$, we decompose $g$ as

$$
h(z)=\frac{s_{0}}{\left(s_{0}-z\right)} \frac{g\left(s_{0}\right)}{s_{0}}-\frac{g\left(s_{0}\right)-g(z)}{s_{0}-z} .
$$


By Lemmas D.2 and D.3, it is clear that $g$ is a linear combination of moment generating functions. Since $s_{0} /\left(s_{0}-z\right)$ is the moment generating function of the exponential distribution with mean $1 /\left(s_{0}\right)$ and $\left(g\left(s_{0}\right)-g(z)\right) /\left(s_{0}-z\right)$ is the moment generating function of the density $g^{\prime}\left(s_{0}\right) e^{-s_{0} x} \int_{x}^{\infty} e^{s_{0} y} \xi(d y)$ (see the proof of Lemma D.3), it follows from the above decomposition and the no atom assumption that

$$
\tilde{f}(x)=g\left(s_{0}\right) e^{-s_{0} x}-g^{\prime}\left(s_{0}\right) e^{-s_{0} x} \int_{x}^{\infty} e^{s_{0} y} \xi(d y),
$$

which clearly implies (D.4). For $k \geq 2$, we can apply Lemma C.1 since (C.4) is satisfied and $g(z)$ is bounded for $\Re z<s_{0}+\epsilon / 2$. Hence, we get (D.4).

Once we get an asymptotic of the form $x^{\kappa} e^{-\alpha x}$ for a density, we can get the same asymptotic for the corresponding tail probability by the following lemma.

Lemma D.5. Let $p(x)$ be a measurable function on $[0, \infty)$. If $p(x)=$ $b \alpha x^{\kappa} e^{-\alpha x}+o\left(x^{\kappa} e^{-\alpha x}\right)$ as $x \rightarrow \infty$ for some $b, \alpha>0$ and some real number $\kappa$, then

$$
\int_{x}^{\infty} p(u) d u=b x^{\kappa} e^{-\alpha x}+o\left(x^{\kappa} e^{-\alpha x}\right) \quad \text { as } x \rightarrow \infty .
$$

Proof. Let $\epsilon>0$ be fixed. From the assumption on the $p$, there is an $x_{0}>0$ such that

$$
\left|p(u)-b \alpha u^{\kappa} e^{-\alpha u}\right|<\epsilon \alpha u^{\kappa} e^{-\alpha u} \quad \text { for } u \geq x_{0} .
$$

Integrating both sides from $x$ to $\infty$, we have, for any $x \geq x_{0}$,

$$
\left|\int_{x}^{\infty} p(u) d u-b \int_{x}^{\infty} u^{\kappa} \alpha e^{-\alpha u} d u\right|<\epsilon \int_{x}^{\infty} u^{\kappa} \alpha e^{-\alpha u} d u .
$$

Since

$$
\int_{x}^{\infty} u^{\kappa} \alpha e^{-\alpha u} d u=x^{\kappa} e^{-\alpha x}+\int_{x}^{\infty} k u^{\kappa-1} e^{-\alpha u} d u,
$$

and, by l'Hôpital's rule,

$$
\lim _{x \rightarrow \infty} \frac{1}{x^{\kappa} e^{-\alpha x}} \int_{x}^{\infty} k u^{\kappa-1} e^{-\alpha u} d u=\lim _{x \rightarrow \infty} \frac{-k x^{\kappa-1} e^{-\alpha x}}{\left(-\alpha x^{\kappa}+k x^{\kappa-1}\right) e^{-\alpha x}}=0,
$$

it follows from (D.6) that

$$
\lim _{x \rightarrow \infty}\left|\frac{1}{x^{\kappa} e^{-\alpha x}} \int_{x}^{\infty} p(u) d u-b\right|<\epsilon .
$$

This implies (D.5) since $\epsilon>0$ can be arbitrarily small. 
Acknowledgement. We are grateful to Michael Harrison for stimulating discussions and invaluable comments on this paper. This paper was initiated during our stay at Cambridge in 2010 for research program "Stochastic Processes in Communication Sciences". We thank the organizers for inviting us to attend the program and the Isaac Newton Institute for Mathematical Sciences for providing financial support. This research is supported in part by NSF grants CMMI-0727400, CMMI-0825840, CMMI-1030589, and by Japan Society for the Promotion of Science under grant No. 21510165.

\section{REFERENCES}

[1] Avram, F., Dai, J. G., and Hasenbein, J. J. (2001). Explicit solutions for variational problems in the quadrant. Queueing Syst. 37, 1-3, 259-289. MR1833666 (2002e:60037)

[2] Berman, A. and Plemmons, R. J. (1979). Nonnegative matrices in the mathematical sciences. Academic Press [Harcourt Brace Jovanovich Publishers], New York. Computer Science and Applied Mathematics. MR544666 (82b:15013)

[3] Dai, J. G. and Harrison, J. M. (1992). Reflected Brownian motion in an orthant: numerical methods for steady-state analysis. Ann. Appl. Probab. 2, 1, 65-86. MR1143393 (92k:60191)

[4] Dieker, A. B. and Moriarty, J. (2009). Reflected Brownian motion in a wedge: sum-of-exponential stationary densities. Electron. Commun. Probab. 14, 1-16. MR2472171 (2010e:60174)

[5] Doetsch, G. (1974). Introduction to the theory and application of the Laplace transformation. Springer-Verlag, New York. Translated from the second German edition by Walter Nader. MR0344810 (49 \#9549)

[6] Dupuis, P. and Ramanan, K. (2002). A time-reversed representation for the tail probabilities of stationary reflected Brownian motion. Stochastic Process. Appl. 98, 2, 253-287. MR1887536 (2003c:60051)

[7] Dupuis, P. and Williams, R. J. (1994). Lyapunov functions for semimartingale reflecting Brownian motions. Ann. Probab. 22, 2, 680-702. MR1288127 (95k:60204)

[8] Fayolle, G., Iasnogorodski, R., and Malyshev, V. (1999). Random walks in the quarter-plane. Applications of Mathematics (New York), Vol. 40. SpringerVerlag, Berlin. Algebraic methods, boundary value problems and applications. MR1691900 (2000g:60002)

[9] Foley, R. D. and McDonald, D. R. (2005). Bridges and networks: exact asymptotics. Ann. Appl. Probab. 15, 1B, 542-586. MR2114982 (2005m:60209)

[10] Harrison, J. M. (1978). The diffusion approximation for tandem queues in heavy traffic. Adv. in Appl. Probab. 10, 4, 886-905. MR509222 (80a:60101)

[11] Harrison, J. M. and Hasenbein, J. J. (2009). Reflected Brownian motion in the quadrant: tail behavior of the stationary distribution. Queueing Syst. 61, 2-3, 113138. MR2485885 (2010i:60264)

[12] Harrison, J. M. and NGuyen, V. (1993). Brownian models of multiclass queueing networks: current status and open problems. Queueing Systems Theory Appl. 13, 1-3, 5-40. MR1218842 (93m:60185) 
[13] Harrison, J. M. and Williams, R. J. (1987). Brownian models of open queueing networks with homogeneous customer populations. Stochastics 22, 2, 77-115. MR912049 (89b:60215)

[14] Harrison, J. M. and Williams, R. J. (1987). Multidimensional reflected Brownian motions having exponential stationary distributions. Ann. Probab. 15, 1, 115-137. MR877593 (88e:60091)

[15] Hobson, D. G. and Rogers, L. C. G. (1993). Recurrence and transience of reflecting Brownian motion in the quadrant. Math. Proc. Cambridge Philos. Soc. 113, 2, 387399. MR1198420 (93k:60207)

[16] Ignatyuk, I. A., Malyshev, V. A., and Shcherbakov, V. V. (1994). The influence of boundaries in problems on large deviations. Uspekhi Mat. Nauk 49, 2(296), 43-102. MR1283135 (96m:60066)

[17] Kobayashi, M. and Miyazawa, M. (2011). Tail asymptotics of the stationary distribution of a two dimensional reflecting random walk with unbounded upward jumps. Preprint.

[18] Kobayashi, M., Miyazawa, M., and Zhao, Y. Q. (2010). Tail asymptotics of the occupation measure for a Markov additive process with an $M / G / 1$-type background process. Stoch. Models 26, 3, 463-486. MR2739346

[19] Lieshout, P. and Mandjes, M. (2007). Tandem Brownian queues. Math. Methods Oper. Res. 66, 2, 275-298. MR2342215 (2008k:60230)

[20] Lieshout, P. and Mandjes, M. (2008). Asymptotic analysis of Lévy-driven tandem queues. Queueing Syst. 60, 3-4, 203-226. MR2461616 (2009m:60218)

[21] Majewski, K. (1996). Large Deviations of Stationary Reflected Brownian Motions. In Stochastic Networks: Theory and Applications (F. P. Kelly, S. Zachary and I. Ziedins, eds.) Oxford University Press.

[22] Majewski, K. (1998). Large deviations of the steady-state distribution of reflected processes with applications to queueing systems. Queueing Systems Theory Appl. 29, 2-4, 351-381. MR1654452 (2000a:60045)

[23] Markushevich, A. I. (1977). Theory of functions of a complex variable. Vol. I, II, III, English ed. Chelsea Publishing Co., New York. Translated and edited by Richard A. Silverman. MR0444912 (56 \#3258)

[24] Miyazawa, M. (2009). Tail decay rates in double QBD processes and related reflected random walks. Math. Oper. Res. 34, 3, 547-575. MR2555336 (2011c:60233)

[25] Miyazawa, M. and Kobayashi, M. (2011). Conjectures on tail asymptotics of the stationary distribution for a multidimensional SRBM. Queueing Systems. to appear.

[26] Miyazawa, M. and Rolski, T. (2009). Tail asymptotics for a Lévy-driven tandem queue with an intermediate input. Queueing Syst. 63, 1-4, 323-353. MR2576017 (2011h:60191)

[27] Reiman, M. I. and Williams, R. J. (1988). A boundary property of semimartingale reflecting Brownian motions. Probab. Theory Related Fields 77, 1, 87-97. MR921820 (89a:60191)

[28] Rockafellar, R. T. (1970). Convex analysis. Princeton Mathematical Series, No. 28. Princeton University Press, Princeton, N.J. MR0274683 (43 \#445)

[29] Taylor, L. M. and Williams, R. J. (1993). Existence and uniqueness of semimartingale reflecting Brownian motions in an orthant. Probab. Theory Related Fields 96, 3, 283-317. MR1231926 (94m:60161) 
[30] Williams, R. J. (1995). Semimartingale reflecting Brownian motions in the orthant. In Stochastic networks. IMA Vol. Math. Appl., Vol. 71. Springer, New York, 125-137. MR1381009 (96k:60213)

[31] Williams, R. J. (1996). On the approximation of queueing networks in heavy traffic. In Stochastic Networks: Theory and Applications (F. P. Kelly, S. Zachary and I. Ziedins, eds.). Royal Statistical Society. Oxford University Press.

H. Milton Stewart School of Industrial and Systems ENGINEERING

Georgia Institute of Technology

Atlanta, GA 30332, U.S.A.

E-MAIL: dai@gatech.edu
Department of Information Sciences

TOKYO University of SCIENCE

NodA, Chiba 278-0017, JAPAN

E-MAIL: miyazawa@is.noda.tus.ac.jp 